\title{
CATEGORIFICATION OF QUANTUM SYMMETRIC PAIRS I
}

\author{
HUANCHEN BAO, PENG SHAN, WEIQIANG WANG AND BEN WEBSTER
}

\begin{abstract}
We categorify a coideal subalgebra of the quantum group of $\mathfrak{s l}_{2 r+1}$ by introducing a 2-category analogous to the one defined by Khovanov-Lauda-Rouquier, and show that self-dual indecomposable 1-morphisms categorify the canonical basis of this algebra. This allows us to define a categorical action of this coideal algebra on the categories of modules over cohomology rings of partial flag varieties and on the category $\mathcal{O}$ of type $\mathrm{B} / \mathrm{C}$.
\end{abstract}

\section{Contents}

1. Introduction

1.1. Motivation

1.2. Main results

1.3. Structure of the paper

1.4. Future developments

Acknowledgements

2. Preliminaries on coideal subalgebras

2.1. Notations and conventions

2.2. Reminders on $\dot{\mathbf{U}}$

2.3. The coideal category $\dot{\mathbf{U}}^{3}$

3. Coideal 2-categories

3.1. The 2-category $\widetilde{\mathfrak{U}}^{3}$

3.2. The coideal 2-category $\mathfrak{U}^{3}$

3.3. Symmetries of $\mathfrak{U}^{3}$

4. The Grothendieck group of $\mathfrak{U}^{3}$

4.1. Reformulation of $\jmath$-Serre relations

4.2. Categorification of $\jmath$-Serre (4.1)

4.3. Categorification of $\mathrm{\jmath}$-Serre (4.2)

4.4. Categorification of $\mathrm{J}$-Serre (4.3)-(4.6)

4.5. The Grothendieck group

4.6. Control from the Grothendieck group

5. The Schur 2-category

5.1. Soergel bimodules

5.2. Schur category in type B/C

5.3. Frobenius forms and Demazure operators

5.4. Action of $\mathfrak{U}^{\mathfrak{J}}$ on the Schur 2-category 
6.1. Local fullness of $\Gamma \quad 30$

6.2. Canonical basis $\quad 35$

7. Categorical action on category $\mathcal{O} \quad 36$

7.1. Reminders on Harish-Chandra bimodules $\quad 36$

7.2. The case of types B and C $\quad 38$

Appendix A. Categorification of the $\mathrm{j}$-Serre relations $\quad 39$

A.1. Bubble slides $\quad 39$

\begin{tabular}{lll|}
\hline A.2. Proof of Proposition A.6 & 43 \\
\hline
\end{tabular}

A.3. Proof of the identity (4.11) 46

A.4. Proof of the identity (4.12) 49

References $\quad 52$

\section{INTRODUCTION}

1.1. Motivation. One important development in representation theory in the last decade is the theory of categorical actions of Lie algebras, in the sense of Chuang and Rouquier CR08. The basic object of this theory is a 2-category $\dot{\mathfrak{U}}(\mathfrak{g})$ associated with a semisimple or Kac-Moody Lie algebra $\mathfrak{g}$, introduced independently in two different presentations by Khovanov-Lauda [KL10] and Rouquier [R08] (these were shown to be equivalent by Brundan [Br16]). The notion of a categorical action of $\mathfrak{g}$ is made precise by the structure of 2-morphisms in $\dot{\mathfrak{U}}(\mathfrak{g})$, and it provides an algebraic way of understanding deep structures of the quantum group $\mathbf{U}(\mathfrak{g})$ such as canonical bases [Lu92, Lu94, Ka93].

One case of particular interest is when $\mathfrak{g}=\mathfrak{s l}_{r}$, where Schur duality plays a basic role. The Grothendieck group of $\dot{\mathfrak{U}}\left(\mathfrak{s l}_{r}\right)$ coincides with the modified quantum group $\dot{\mathbf{U}}=\dot{\mathbf{U}}\left(\mathfrak{s l}_{r}\right)$, and the classes of indecomposable 1-morphisms are identified with Lusztig's canonical bases La10, W15. In the quantum setting, Schur duality relates the quantum group $\mathbf{U}\left(\mathfrak{s l}_{r}\right)$ to Hecke algebras of type A [Jim86]. Beilinson-Lusztig-MacPherson [BLM90] constructed the modified quantum group $\dot{\mathbf{U}}\left(\mathfrak{s l}_{r}\right)$ as a limit of a family of finite-dimensional algebras known as Schur algebras, and they realized the Schur algebras using functions on the points of $r$-step partial flag varieties over a finite field. The BLM construction was then adapted in [GL92] to realize quantum Schur duality. This geometric realization of $\dot{\mathbf{U}}\left(\mathfrak{s l}_{r}\right)$ has been lifted to a categorical action of $\dot{\mathfrak{U}}\left(\mathfrak{s l}_{r}\right)$ in KL10. This construction also leads to a categorified Schur algebra, which is intimately related to singular Soergel bimodules [Wil11, MSV13, W15b].

Schur duality has been generalized in [BW13] in connection to the Kazhdan-Lusztig theory for the BGG category $\mathcal{O}$ of type B/C. While other generalizations of Schur duality have changed $\mathfrak{s l}_{r}$ to a different classical Lie algebra [BW89, M87, this construction replaces the Hecke algebra from type A with one of type B/C. Like in type A, this construction admits a BLM-type realization [BKLW], where $\mathbf{U}\left(\mathfrak{s l}_{r}\right)$ is replaced by the coideal algebra $\mathbf{U}^{\jmath}$ associated with the Cartan involution composed with the diagram involution of the $\mathrm{A}_{2 r}$ Dynkin diagram. Note that $\left(\mathbf{U}\left(\mathfrak{s l}_{2 r+1}\right), \mathbf{U}^{\jmath}\right)$ forms a quantum symmetric pair in the sense of [Le03]. The commutant of the Hecke algebra of type B/C in this setting is called jSchur algebra. They are naturally quotients of $\mathbf{U}^{3}$, and admit a geometric realization in terms of partial flag varieties of type B/C 
over finite fields. The idempotented coideal algebra can be realized as a limit of the family of jSchur algebras. Based on this construction, it was shown in [LW15] that $\dot{\mathbf{U}}^{\text {J admits a }}$ canonical basis with desirable positivity properties, analogous to Lusztig's canonical basis for Uं as defined in [Lu94, Chapter 25]. The $\mathcal{A}=\mathbb{Z}\left[q, q^{-1}\right]$-span of this basis gives an integral form ${ }_{\mathcal{A}} \dot{\mathrm{U}}^{\jmath}$.

By analogy with the "weak $\mathfrak{s l}_{2}$-categorifications" discussed in [CR08, 5.1], we can think of the construction of [BW13] as a weak categorical action of $\mathbf{U}^{\jmath}$. The construction of [BKLW] shows the existence of a similar weak action of $\mathbf{U}^{\jmath}$ on constructible sheaves on partial flag varieties of type B/C. Following the analogy with type A, it is natural to expect that we can define a strong categorical action of $\mathbf{U}^{3}$ by a 2 -category $\dot{\mathfrak{U}}^{\jmath}$ analogous to $\dot{\mathfrak{U}}$.

The existence of this 2-category together with its basic 2-representations is the principal result of this paper.

1.2. Main results. To explain the definition of our 2-category $\dot{\mathfrak{U}}^{3}$, let us first give a description of $\dot{\mathbf{U}}^{\jmath}$. The idempotented algebra $\dot{\mathbf{U}}^{\jmath}$ can be viewed as a category with objects indexed by a weight lattice $\mathrm{X}_{\jmath}$ and morphisms generated by

$$
\mathcal{E}_{i}: \lambda \rightarrow \lambda+\alpha_{i} \quad \text { and } \quad \mathcal{F}_{i}: \lambda \rightarrow \lambda-\alpha_{i}, \quad \text { for } i=\diamond, \diamond+1, \ldots, r-\diamond \text { with } \diamond=\frac{1}{2} .
$$

See Section 2.3 for a precise definition. For $i>\diamond$, the generators $\mathcal{E}_{i}, \mathcal{F}_{i}$ satisfy the same relations as standard Chevalley generators in $\dot{\mathbf{U}}$. However, the relations are different when $i=\diamond$ : there is no relation between length 2 monomials in $\mathcal{E}_{\diamond}$ and $\mathcal{F}_{\diamond}$. Instead they satisfy inhomogeneous $\jmath$-Serre relations (2.9) and (2.10), whose summands are no longer canonical basis elements. In particular, $\mathbf{U}^{\jmath}$ does not have a triangular decomposition like that of $\mathbf{U}$.

To define $\dot{\mathfrak{U}}^{3}$, we follow the approach of [La10, KL10]: we already know of a weak $\dot{\mathfrak{U}}^{3}$-action on the modules over cohomology rings of partial flag varieties, so we can extract the relations in $\dot{\mathfrak{U}}^{3}$ based on computations in this category. While the 2-morphisms acting on $\mathcal{E}_{i}$ and $\mathcal{F}_{i}$ for $i \neq \diamond$ satisfy the same relations as in $\dot{\mathfrak{U}}$, new relations appear for $i=\diamond$.

One can think of these new relations as combining the KLR relations for the elements $\left\{E_{\diamond}, E_{-\diamond}\right\}$ in $\mathbf{U}^{+}\left(\mathfrak{s l}_{3}\right)$ and for $\left\{E_{\diamond}, F_{\diamond}\right\}$ in $\mathbf{U}\left(\mathfrak{s l}_{2}\right)$. In particular, the usual bicross relations which categorify the commutator relation for $\mathcal{E}_{i}$ and $\mathcal{F}_{i}(3.12)-(3.13)$ combine with (3.5) to give (3.14)-(3.15). Similarly, there is a new relation involving a triple point (3.17) which combines the relation of [La10, Proposition 5.8] with (3.6); this relation is used in proving the categorified $\mathrm{\jmath}$-Serre relations, as we will explain in Section 4 and Appendix A, The bubble relations and bubble slide formulas for $i=\diamond$ here are also somewhat different from [La10].

The complexity of this last triple point relation makes it difficult to verify the relations of $\dot{\mathfrak{U}}^{\jmath}$ directly (much like the presentation of $\dot{\mathfrak{U}}$ in [KL10]). However, we show that if $\mathcal{E}_{i}$ and $\mathcal{F}_{i}$ satisfy the relations of the coideal subalgebra at the decategorified level, then (3.17) is forced by the other relations in $\dot{\mathfrak{U}}^{\jmath}$; see Proposition 4.10. The reader can think of Proposition 4.10 as a weak version of "control from $K_{0}$ " theorems, such as [R08, 5.27].

Now, let us describe the main results of this paper. The first is that $\dot{\mathfrak{U}}^{\jmath}$ is a categorification of $\dot{\mathbf{U}}^{\jmath}$, in the same sense as the relationship of $\dot{\mathfrak{U}}$ and $\dot{\mathbf{U}}$. 
Theorem A (Theorem 6.5). There is an algebra isomorphism between the Grothendieck group of $\dot{\mathfrak{U}}^{\mathfrak{l}}$ and ${ }_{\mathcal{A}} \dot{\mathbf{U}}^{3}$, with indecomposable self-dual 1-morphisms matching the canonical basis of ${ }_{\mathcal{A}} \dot{\mathbf{U}}^{3}$.

To prove this theorem, and as evidence for the usefulness of this categorification, we ask for generalizations of the categorical actions of $\mathfrak{U}$ discussed earlier. Let $\mathrm{G}$ be either the group $\mathrm{SO}(2 m+1)$ or $S p(2 m)$, and let $\mathfrak{g}$ be its Lie algebra. One can define a 2-category $\mathfrak{F}_{r, m}$ analogous to the "flag category" of Khovanov and Lauda [KL10] using cohomology rings of partial flag varieties of $\mathrm{G}$, see Section 5.2 for the precise definition. The 2-category $\dot{\mathfrak{U}}^{3}$ admits the following categorical actions.

Theorem B (Theorem [5.8, Theorem 6.1, Proposition 6.3, Theorem 7.6).

(a) There is a functor $\Gamma: \dot{\mathfrak{U}} \rightarrow \mathfrak{F}_{r, m}$ for each $m$. This functor is essentially surjective on 1-morphisms (and becomes full on 2-morphisms after a small modification).

(b) The category $\mathfrak{U}^{\mathfrak{j}}$ acts on the category $\mathcal{O}$ of $\mathfrak{g}$ such that an object $\lambda \in \mathrm{X}_{\mathrm{J}}$ is sent to a block of $\mathcal{O}$, and $\mathcal{E}_{i}, \mathcal{F}_{i}$ act by translation functors on blocks.

The actions in Parts (a) and (b) provide the desired enhancements of the weak categorical actions in BKLW] and in BW13, respectively.

These actions are related via the classic links between projective functors, Harish-Chandra bimodules and singular Soergel bimodules given by [Soe90, Soe92, Str04] and [Wil11]. The action of Part (a) allows us to show that $\dot{\mathfrak{U}}^{\mathfrak{j}}$ acts on any category of representations of Lie algebra of type $\mathrm{B} / \mathrm{C}$ which are of finite length and are closed under tensor product with finite dimensional modules (see Theorem [7.6). Category $\mathcal{O}$ is an example of such a category, as are many of the variations on it.

1.3. Structure of the paper. In Section 2 we will recall the basic structure results on the algebras $\mathbf{U}$ and $\mathbf{U}^{3}$, along with their idempotented forms $\dot{\mathbf{U}}$ and $\dot{\mathbf{U}}^{3}$. In Section 3 , we give the definition of the 2-category $\mathfrak{U}^{3}$, presented in diagrammatic terms, and study some basic properties of it. In Section 4, we precisely formulate the categorification of the $\jmath$-Serre relations, which allows us to define a surjective algebra homomorphism $\dot{\mathbf{U}}^{\jmath} \rightarrow K_{0}\left(\dot{\mathfrak{U}}^{\mathfrak{j}}\right)$. The categorification of $\jmath$-Serre relations involves some lengthy diagrammatic computation, which will be given in Appendix A. We construct the 2-functor $\Gamma: \dot{\mathfrak{U}}^{j} \rightarrow \mathfrak{F}_{r, m}$ in Section 5, and prove it is locally full in Section 6. This allows us to deduce the isomorphism $\dot{\mathbf{U}}^{\jmath} \simeq K_{0}\left(\dot{\mathfrak{U}}^{\mathfrak{l}}\right)$ and the matching of

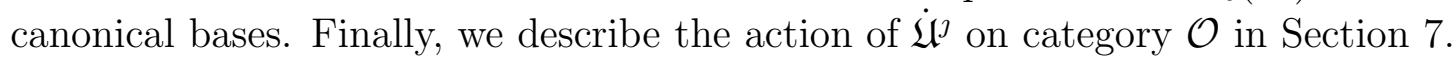

1.4. Future developments. The construction of this paper seems likely to be only one of a family of categorifications corresponding to coideal subalgebras, and relevant to the representation theory of classical groups and Lie algebras of types $B / C / D$ in many contexts. Whereas categorical actions of any Kac-Moody algebra are built from categorical $\mathfrak{s l}_{2}$-actions, actions of $\dot{\mathfrak{U}}^{\mathfrak{j}}$ include a new basic building block, which may be useful in other contexts. For reasons of space and complexity, we have left the consideration of several natural questions that arise in this framework to future work. 
In a sequel to this work, we will consider analogues of cyclotomic KLR algebras for $\mathfrak{U}^{j}$; these are algebras which naturally categorify the restrictions to $\mathbf{U}^{\jmath}$ of finite-dimensional simple $\mathbf{U}$ modules and their ycanonical bases defined in [BW13. We will also study the relationship between $\dot{\mathfrak{U}}^{\jmath}$ and $\dot{\mathfrak{U}}$ which can be regarded as a categorical quantum symmetric pair.

The connection between the coideal algebras and category $\mathcal{O}$ of type $\mathrm{D}$ has been independently observed in ES13], where they also studied relations between morphisms in $\mathcal{O}$ with Nazarov-Wenzl algebras and affine Brauer algebras. We expect the categorical action of our 2-category $\dot{\mathfrak{U}}^{\jmath}$ will bring a new perspective on these algebras. Note that there should also be a type D analogue of our main results; see [B16] for Kazhdan-Lusztig theory of type D and [FL15] for geometric Schur duality of type D.

The algebra $\mathbf{U}^{\top}$ is merely a special example of the coideal algebras arising from quantum symmetric pairs [Le03, Ko14]. Particularly important special cases include the coideal subalgebra $\mathbf{U}^{\imath} \subset \mathbf{U}\left(\mathfrak{s l}_{2 r}\right)$ associated to its diagram automorphism, and the affine analogues of these algebras, attached to diagram automorphisms for $\widehat{\mathfrak{s l}}_{n}$ [FLLLW]. The former also appears in geometric Schur duality and the study of category $\mathcal{O}$ in type B/C [BW13, BKLW, LW15] while the latter is expected to play a fundamental role in the study of modular representations of type B/C. These other symmetric pairs will require more work to categorify. Since these algebras admit natural geometric realizations and canonical bases, the techniques of this paper can likely be applied to them as well.

Acknowledgements. H.B. is partially supported by an AMS-Simons travel grant. P.S. is partially supported by the ANR grant number ANR-12-JS01-0003, ANR-13-BS01-0001-01. W.W. is partially supported by the NSF grant DMS-1405131. B.W. is partially supported by the NSF grant DMS-1151473 and the Alfred P. Sloan Foundation. We thank the Institute of Mathematical Science at the University of Virginia, Institute of Mathematics, Academia Sinica (Taipei), Yau Mathematical Sciences Center at Tsinghua University, and Max Planck Institute for Mathematics for support which facilitated this collaboration. We thank Zajj Daugherty, Aaron Lauda, Yiqiang Li, Marco Mackaay, Arun Ram and Catharina Stroppel for useful conversations. We thank the referee for a careful reading and corrections on the proof of Lemma A.1.

\section{Preliminaries on Coideal subalgebras}

2.1. Notations and conventions. Let $q$ be a formal variable and let $\mathcal{A}=\mathbb{Z}\left[q, q^{-1}\right]$. For $a \in \mathbb{Z}$, let

$$
[a]=\frac{q^{a}-q^{-a}}{q-q^{-1}} \quad \text { and } \quad[a] !=[1][2] \cdots[a] \quad \text { for } a \geqslant 0 .
$$

The $a$-th divided power of an element $E$ in a $\mathbb{Q}(q)$-algebra is the element $E^{(a)}:=E^{a} /[a]$ !.

Let $\mathcal{C}$ be an additive category. For any object $x$ in $\mathcal{C}$, we denote by $1_{x}$ the identity endomorphism of $x$. A grading on $\mathcal{C}$ is an auto-equivalence $\{1\}: \mathcal{C} \rightarrow \mathcal{C}$. Let $\{\ell\}$ denote the $\ell$-fold composition of the auto-equivalence $\{1\}$. Given an object $x$ in $\mathcal{C}$ and $f=\sum f_{s} q^{s} \in \mathcal{A}$, we write $\oplus_{f} x$ or $x^{\oplus f}$ for the direct sum over $s$ of $f_{s}$ copies of $x\{s\}$. The split Grothendieck group $K_{0}(\mathcal{C})$ is the abelian group generated by symbols $[x]$ for $x$ an object, with the relation $[x \oplus y]=[x]+[y]$. A grading induces an $\mathcal{A}$-module on $K_{0}(\mathcal{C})$ such that $q[x]=[x\{1\}]$. Given two objects $x, y$, let $\operatorname{Hom}^{s}(x, y)$ denote the space $\operatorname{Hom}(x\{s\}, y)$ of morphisms of degree $s$. We 
abbreviate $\operatorname{Hom}^{\bullet}(x, y)=\bigoplus_{s \in \mathbb{Z}} \operatorname{Hom}^{s}(x, y)$. The idempotent completion (or Karoubi envelope) $\dot{\mathcal{C}}$ is the category whose objects are pairs $(M, e)$ of $M \in \mathrm{Ob}(\mathcal{C})$ and idempotent endomorphisms $e: M \rightarrow M$, with $(M, e)$ serving as the image of the idempotent $e$. The category $\dot{\mathcal{C}}$ is universal among idempotent complete categories with a functor from $\mathcal{C}$.

Given a ring $\mathbf{k}$, a graded $\mathbf{k}$-linear 2-category $\mathfrak{A}$ is a category enriched over graded additive k-linear categories, that is, a 2-category such that the $\mathcal{H o m}$ categories are graded additive k-linear categories and the composition maps $\mathcal{H o m}_{\mathfrak{A}}(x, y) \times \mathcal{H}_{\mathfrak{A}}(y, z) \rightarrow \mathcal{H}_{\mathfrak{A}}\left(m_{\mathfrak{A}}(x, z)\right.$ form a graded additive $\mathbf{k}$-linear functor. We will abbreviate $\mathfrak{A}(x, y)=\mathcal{H}_{\mathfrak{A}}(x, y)$. Objects and morphisms in $\mathfrak{A}(x, y)$ will be respectively called 1-morphisms and 2-morphisms in $\mathfrak{A}$. A 2category is called strict if 1-morphisms compose strictly associatively, and a 2-functor is called strict when it preserves compositions of 1-morphisms on the nose. We will always assume that $\mathfrak{A}(x, x)$ has a unit object, denoted by $1_{x}$. The Grothendieck group of $\mathfrak{A}$ is the category $K_{0}(\mathfrak{A})$ with same objects as $\mathfrak{A}$, and with morphisms given by the Grothendieck group of the corresponding $\mathcal{H}$ om-category. If $\mathfrak{A}$ is graded, then $K_{0}(\mathfrak{A})$ is an $\mathcal{A}$-linear category.

Recall that a functor is essentially surjective if any object in the target is isomorphic to an object in the image. It is full/faithful if it is surjective/injective on Hom sets. A fully faithful, essentially surjective functor is an equivalence. A 2-functor $\Phi: \mathfrak{A} \rightarrow \mathfrak{B}$ is locally full (resp. faithful, essentially surjective) if the induced functors $\mathfrak{A}(x, y) \rightarrow \mathfrak{B}(\Phi(x), \Phi(y)$ ) is full (resp. faithful, essentially surjective) for all objects $x, y \in \mathfrak{A}$.

2.2. Reminders on $\dot{U}$. Fix a positive integer $r$ and define

$$
\mathbb{I}=\mathbb{I}_{2 r}=\left\{i \in \mathbb{Z}+\frac{1}{2} \mid-r<i<r\right\} .
$$

Due to an extensive use of $\frac{1}{2}$ throughout the paper, we will often write

$$
\diamond=\frac{1}{2} \text {. }
$$

Consider the root datum of type $A_{2 r}$ with Cartan matrix indexed by $\mathbb{I}$, weight lattice X, simple roots $\left\{\alpha_{i}\right\}_{i \in \mathbb{I}} \subset \mathrm{X}$, simple coroots $\left\{\alpha_{i}^{\vee}\right\}_{i \in \mathbb{I}}$, and the coroot lattice $\mathrm{Y}=\bigoplus_{i \in \mathbb{I}} \mathbb{Z} \alpha_{i}^{\vee}$. There is a perfect pairing

$$
\langle\cdot, \cdot\rangle: \mathrm{Y} \times \mathrm{X} \longrightarrow \mathbb{Z}
$$

The entries of the Cartan matrix are given by $\left\langle\alpha_{i}^{\vee}, \alpha_{j}\right\rangle$ for $i, j \in \mathbb{I}$.

Consider the lattice $\bigoplus_{a=-r}^{r} \mathbb{Z} \varepsilon_{a}$ with the standard pairing $\left\langle\varepsilon_{a}, \varepsilon_{b}\right\rangle=\delta_{a, b}$. We will identify

$$
\mathrm{X}=\bigoplus_{a=-r}^{r} \mathbb{Z} \varepsilon_{a} / \mathbb{Z}\left(\sum_{a=-r}^{r} \varepsilon_{a}\right) .
$$

Then $\alpha_{i}=\varepsilon_{i-\diamond}-\varepsilon_{i+\diamond}\left(\bmod \mathbb{Z} \sum_{a=-r}^{r} \varepsilon_{a}\right)$ lies in X.

The quantum group $\mathbf{U}=\mathbf{U}_{q}\left(\mathfrak{s l}_{2 r+1}\right)$ is the $\mathbb{Q}(q)$-algebra generated by $E_{i}, F_{i}, K_{i}^{ \pm 1}, i \in \mathbb{I}$ subject to the relations

$$
\begin{aligned}
K_{i} K_{j}=K_{j} K_{i}, & K_{i} E_{j} K_{i}^{-1}=q^{\left\langle\alpha_{i}^{\vee}, \alpha_{j}\right\rangle} E_{j}, \\
{\left[E_{i}, F_{j}\right]=\delta_{i j} \frac{K_{i}-K_{i}^{-1}}{q-q^{-1}}, } & K_{i} F_{j} K_{i}^{-1}=q^{-\left\langle\alpha_{i}^{\vee}, \alpha_{j}\right\rangle} F_{j},
\end{aligned}
$$


and the quantum Serre relations (see (2.3) below). We may write $\mathbf{U}=\mathbf{U}_{2 r+1}$ if necessary. It is a Hopf algebra, with a coproduct $\Delta: \mathbf{U} \rightarrow \mathbf{U} \otimes \mathbf{U}$ such that

$$
\Delta\left(K_{i}\right)=K_{i} \otimes K_{i}, \quad \Delta\left(E_{i}\right)=1 \otimes E_{i}+E_{i} \otimes K_{i}^{-1}, \quad \Delta\left(F_{i}\right)=F_{i} \otimes 1+K_{i} \otimes F_{i}, \forall i .
$$

There is a unique involution (called bar involution) $\psi: \mathbf{U} \rightarrow \mathbf{U}$ as $\mathbb{Q}$-algebra which sends $q$ to $q^{-1}, K_{i}$ to $K_{i}^{-1}$ and fixes $E_{i}, F_{i}$, for all $i$.

We will be interested in an idempotented (or modified) form of $\mathbf{U}$ introduced by Lusztig Lu94. Consider the $\mathbb{Q}(q)$-linear category $\dot{U}$ with the object set $\mathrm{X}$ and morphisms generated by $E_{i}: \lambda \rightarrow \lambda+\alpha_{i}, F_{i}: \lambda \rightarrow \lambda-\alpha_{i}$, subject to the relations

$$
\begin{aligned}
{\left[E_{i}, F_{j}\right] 1_{\lambda} } & =\delta_{i j}\left[\left\langle\alpha_{i}^{\vee}, \lambda\right\rangle\right] 1_{\lambda}, \\
\sum_{a+b=1-\left\langle\alpha_{i}^{\vee}, \alpha_{j}\right\rangle}(-1)^{a} E_{i}^{(a)} E_{j} E_{i}^{(b)} & =\sum_{a+b=1-\left\langle\alpha_{i}^{\vee}, \alpha_{j}\right\rangle}(-1)^{a} F_{i}^{(a)} F_{j} F_{i}^{(b)}=0, \quad \forall i \neq j .
\end{aligned}
$$

Lusztig's idempotented algebra (also denoted by $\mathbf{U}$ ) can be identified with the direct sum of all Hom-spaces in this category. Let ${ }_{\mathcal{A}} \mathbf{U}$ be the $\mathcal{A}$-linear subcategory of $\dot{\mathbf{U}}$ with the same objects and with morphisms generated over $\mathcal{A}$ by $E_{i}^{(a)}, F_{i}^{(a)}$ for $i \in \mathbb{I}, a \geqslant 0$. This corresponds to Lusztig's integral $\mathcal{A}$-form of $\dot{U}$.

\subsection{The coideal category $\dot{\mathbf{U}}^{\jmath}$. Set}

$$
\mathbb{I}^{\jmath}=\mathbb{I}_{r}^{\jmath}=\mathbb{I} \cap \mathbb{R}_{>0}=\{\diamond, \diamond+1, \ldots, \diamond+r-1\} .
$$

We shall consider a $\mathbb{Q}(q)$-algebra $\mathbf{U}^{\jmath}$ which admits a presentation with generators $\mathcal{E}_{i}, \mathcal{F}_{i}, \mathcal{K}_{i}^{ \pm 1}$ for $i \in \mathbb{I}^{\jmath}$, and a set of relations; see [BW13, Section 6.1]. There is an embedding of algebras $\jmath: \mathbf{U}^{\jmath} \rightarrow \mathbf{U}$ such that

$$
\mathcal{E}_{i} \mapsto E_{i}+K_{i}^{-1} F_{-i}, \quad \mathcal{F}_{i} \mapsto F_{i} K_{-i}^{-1}+E_{-i}, \quad \mathcal{K}_{i} \mapsto K_{i} K_{-i}^{-1}, \quad \forall i \in \mathbb{I}^{\jmath} .
$$

We will consider the algebra $\mathbf{U}^{\jmath}$ as a subalgebra of $\mathbf{U}$ under the embedding J. We may write $\mathbf{U}^{\jmath}=\mathbf{U}_{r}^{\jmath}$ if necessary.

The subalgebra $\mathbf{U}^{\jmath}$ has a further compatibility with the coproduct $\Delta$ : it is a coideal subalgebra of $\mathbf{U}$. That is, the coproduct $\Delta$ on $\mathbf{U}$ restricts to a $\mathbb{Q}(q)$-algebra homomorphism $\Delta: \mathbf{U}^{\jmath} \rightarrow \mathbf{U}^{\jmath} \otimes \mathbf{U}$. The specialization of $\mathbf{U}^{\jmath}$ at $q=1$ is just the enveloping algebra of $\mathfrak{s l}_{2 r+1}^{\vartheta}$, the Lie subalgebra fixed by the involution $\vartheta: \mathfrak{s l}_{2 r+1} \rightarrow \mathfrak{s l}_{2 r+1}$ given by rotating $(2 r+1) \times(2 r+1)$ matrices by $\pi$ radians. Hence $\left(\mathbf{U}, \mathbf{U}^{\jmath}\right)$ is an example of a quantum symmetric pair [Le03, Ko14].

Let $\theta$ be the involution of the lattice $\mathrm{X}$ induced by letting $\theta\left(\varepsilon_{a}\right)=-\varepsilon_{-a}$ for $-r \leqslant a \leqslant r$. We may write $\lambda^{\theta}=\theta(\lambda)$, for $\lambda \in \mathrm{X}$. Denote by $\mathrm{X}^{\theta}$ the sublattice of $\theta$-fixed points in $\mathrm{X}$. Note that $\alpha_{i}^{\theta}=\alpha_{-i}$ for all $i \in \mathbb{I}$, hence $\theta$ induces an automorphism of the root system. Set ${ }^{\theta} \alpha_{i}^{\vee}=\alpha_{i}^{\vee}-\alpha_{-i}^{\vee}$ for $i \in \mathbb{I}^{\jmath}$. Let

$$
\mathrm{X}_{\jmath}=\mathrm{X} / \mathrm{X}^{\theta}, \quad \mathrm{Y}^{\jmath}=\bigoplus_{i \in \mathbb{I}^{\jmath}} \mathbb{Z}^{\theta} \alpha_{i}^{\vee} .
$$

The lattice $\mathrm{X}_{\jmath}$ can be regarded as a weight lattice for $\mathrm{U}^{\jmath}$. The pairing (2.2) induces a nondegenerate pairing

$$
\langle\cdot, \cdot\rangle: \mathrm{Y}^{\jmath} \times \mathrm{X}_{\jmath} \longrightarrow \mathbb{Z}
$$


For $\lambda \in \mathrm{X}_{\jmath}$, we write

$$
\lambda_{i}=\left\langle{ }^{\theta} \alpha_{i}^{\vee}, \lambda\right\rangle, \quad \text { for } i \in \mathbb{I}^{\jmath} .
$$

For $\lambda \in \mathrm{X}$ we will denote its image in $\mathrm{X}_{\jmath}$ again by $\lambda$ if there is no confusion. In particular, we will often regard $\alpha_{i} \in \mathrm{X}$ for $i \in \mathbb{I}^{\jmath}$ as an element in $\mathrm{X}_{\jmath}$. Note the unusual pairing

$$
\left\langle{ }^{\theta} \alpha_{\diamond}^{\vee}, \alpha_{\diamond}\right\rangle=3 \text {. }
$$

Let $\dot{\mathbf{U}}^{\jmath}$ be the $\mathbb{Q}(q)$-linear category with the object set $\mathrm{X}_{\jmath}$ and morphisms generated by $\mathcal{E}_{i}: \lambda \mapsto \lambda+\alpha_{i}=\lambda-\alpha_{-i}, \mathcal{F}_{i}: \lambda \mapsto \lambda-\alpha_{i}=\lambda+\alpha_{-i}$, for all $i \in \mathbb{I}^{\jmath}$, subject to the following relations for $i \neq j$ :

$$
\begin{aligned}
{\left[\mathcal{E}_{i}, \mathcal{F}_{j}\right] 1_{\lambda} } & =0, \\
{\left[\mathcal{E}_{i}, \mathcal{F}_{i}\right] 1_{\lambda} } & =\left[\lambda_{i}\right] 1_{\lambda}, \quad \forall i \neq \diamond, \\
\sum_{a+b=1-\left\langle\alpha_{i}^{\vee}, \alpha_{j}\right\rangle}(-1)^{a} \mathcal{E}_{i}^{(a)} \mathcal{E}_{j} \mathcal{E}_{i}^{(b)} & =\sum_{a+b=1-\left\langle\alpha_{i}^{\vee}, \alpha_{j}\right\rangle}(-1)^{a} \mathcal{F}_{i}^{(a)} \mathcal{F}_{j} \mathcal{F}_{i}^{(b)}=0, \\
\left(\mathcal{E}_{\diamond}^{(2)} \mathcal{F}_{\diamond}-\mathcal{E}_{\diamond} \mathcal{F}_{\diamond} \mathcal{E}_{\diamond}+\mathcal{F}_{\diamond} \mathcal{E}_{\diamond}^{(2)}\right) 1_{\lambda} & =-\left(q^{\lambda_{\diamond}+2}+q^{-\lambda_{\diamond}-2}\right) \mathcal{E}_{\diamond} 1_{\lambda}, \\
\left(\mathcal{F}_{\diamond}^{(2)} \mathcal{E}_{\diamond}-\mathcal{F}_{\diamond} \mathcal{E}_{\diamond} \mathcal{F}_{\diamond}+\mathcal{E}_{\diamond} \mathcal{F}_{\diamond}^{(2)}\right) 1_{\lambda} & =-\left(q^{\lambda_{\diamond}-1}+q^{-\lambda_{\diamond}+1}\right) \mathcal{F}_{\diamond} 1_{\lambda} .
\end{aligned}
$$

The definition of $\dot{\mathbf{U}}^{\jmath}$ here is basically the same as the one in [LW15] but differs from the one in [BKLW] which used a different object set. Note that a major difference in the presentation of $\dot{\mathbf{U}}^{\jmath}$ with respect to $\dot{\mathbf{U}}$ is that there is no relation between monomials of length $\leq 2$ in $\mathcal{E}_{\diamond}$, $\mathcal{F}_{\diamond}$, but rather they satisfy the inhomogeneous relations (2.9), (2.10), which will be referred as ग-Serre relations. Note also that the algebra $\mathbf{U}^{\jmath}$ or $\dot{\mathbf{U}}^{\jmath}$ does not have any natural triangular decomposition due to the mixture of $\mathcal{E}_{\diamond}$ and $\mathcal{F}_{\diamond}$ in these $\gamma$-Serre relations.

Let ${ }_{\mathcal{A}} \dot{\mathbf{U}}^{\jmath}$ be the $\mathcal{A}$-linear subcategory of $\dot{\mathbf{U}}^{\jmath}$ with the same objects and with morphisms generated by divide powers $\mathcal{E}_{i}^{(a)} 1_{\lambda}, \mathcal{F}_{i}^{(a)} 1_{\lambda}$, for all $i \in \mathbb{I}^{\jmath}, \lambda \in \mathrm{X}_{\jmath}$, and $a \geq 0$. It was shown in BKLW, LW15] that ${ }_{\mathcal{A}} \dot{\mathbf{U}}^{\jmath}$ is a free $\mathcal{A}$-module and $\mathbb{Q}(q) \otimes_{\mathcal{A}{ }_{\mathcal{A}} \dot{\mathbf{U}}^{\jmath}}=\dot{\mathbf{U}}^{\jmath}$.

Denote by $\varpi$ the unique element in $\mathrm{X}_{\jmath}$ such that

$$
\varpi_{\diamond}=1, \quad \varpi_{i}=0 \quad\left(i \in \mathbb{I}^{\jmath} \backslash\{\diamond\}\right) .
$$

The following are the idempotented counterparts of the symmetries for $\mathbf{U}^{\jmath}$ given in $[\mathrm{BW} 13$, Lemma 6.1].

\section{Lemma 2.1.}

(a) There exists an involution $\omega_{\text {j }}$ on $\dot{\mathbf{U}}^{3}$ as a $\mathbb{Q}(q)$-algebra which sends $1_{\lambda}$ to $1_{-\lambda-\varpi}$ and switches $\mathcal{E}_{i}$ with $\mathcal{F}_{i}$, for all $i$ and $\lambda \in \mathrm{X}_{\jmath}$.

(b) There exists an anti-involution $\sigma_{\jmath}$ on $\dot{\mathbf{U}}^{\jmath}$ as a $\mathbb{Q}(q)$-algebra which sends $1_{\lambda}$ to $1_{-\lambda-\varpi}$ and fixes $\mathcal{E}_{i}, \mathcal{F}_{i}$, for all $i$ and $\lambda \in \mathrm{X}_{\jmath}$.

(c) There exists an involution (called bar involution) $\psi_{\jmath}$ on $\dot{\mathbf{U}}^{\jmath}$ as a $\mathbb{Q}$-algebra which sends $q$ to $q^{-1}$, and fixes $1_{\lambda}, \mathcal{E}_{i}, \mathcal{F}_{i}$, for all $i$ and $\lambda \in \mathrm{X}_{\jmath}$.

In particular, all three (anti-)involutions $\omega_{\jmath}, \sigma_{\jmath}$ and $\psi_{\jmath}$ preserve the $\mathcal{A}$-form ${ }_{\mathcal{A}} \dot{\mathbf{U}}^{\jmath}$.

Proof. Follows by inspection of the defining relations for $\dot{\mathbf{U}}^{\jmath}$ in $(2.6)-(2.10)$. 


\section{Coideal 2-CATEgories}

In this section, we introduce the main object of this paper, the 2-category $\mathfrak{U}^{\jmath}$ (see Definition 3.3 . This is an analogue of the category $\mathfrak{U}$ introduced in [KL10, Section 5.1] (denoted by $\mathcal{U}$ therein). Recall that $\mathfrak{U}$ is a strict graded additive $\mathbf{k}$-linear 2-category with object set $\mathrm{X}$. Its 1-morphisms are generated by

$$
E_{i}: \lambda \rightarrow \lambda+\alpha_{i}, \quad F_{i}: \lambda \rightarrow \lambda-\alpha_{i} \quad(i \in \mathbb{I}),
$$

with generating 2-morphisms given by diagrams modulo certain diagrammatic relations. We will not reproduce these here, since they appear in the relations (3.1 3.16) below (with ${ }^{\theta} \alpha_{i}^{\vee}$ everywhere replaced by $\alpha_{i}^{\vee}$ ) which do not involve $i=\diamond$.

We start by introducing a weak version $\widetilde{\mathfrak{U}}^{3}$ of this 2-category, which lacks the most difficult relation to verify. This notion is useful since we will show later that a representation of $\widetilde{\mathfrak{U}}^{\jmath}$ satisfying certain conditions on its Grothendieck group descends to an action of $\mathfrak{U}^{\mathfrak{J}}$ (Proposition 4.10).

3.1. The 2-category $\widetilde{\mathfrak{U}}^{j}$. Fix a commutative ring $\mathbf{k}$ with identity such that 2 is a unit. For $i, j \in \mathbb{I}^{\jmath}$ we set

$$
t_{i j}= \begin{cases}-1, & \text { if } j=i-1 \\ 1, & \text { otherwise }\end{cases}
$$

Definition 3.1. The weak coideal 2-category $\widetilde{\mathfrak{U}}^{\jmath}$ is the strict graded additive $\mathbf{k}$-linear 2-category with objects $\lambda$ for all $\lambda \in \mathrm{X}_{\jmath}$. The 1-morphisms are generated by

$$
\mathcal{E}_{i}: \lambda \rightarrow \lambda+\alpha_{i}, \quad \mathcal{F}_{i}: \lambda \rightarrow \lambda-\alpha_{i}, \quad \text { for } i \in \mathbb{I}^{\jmath} .
$$

Here "generated" means taking all the direct sums of compositions of shifts of these 1-morphisms. The generating 2-morphisms are presented by diagrams: denote the identity 2-morphisms of $\mathcal{E}_{i} 1_{\lambda}$ and $\mathcal{F}_{i} 1_{\lambda}$ by ${ }^{\lambda+\alpha_{i} \uparrow}{ }_{i} \lambda$ and $\underset{i}{\lambda-\alpha_{i} \downarrow \lambda}$, and the other generators are

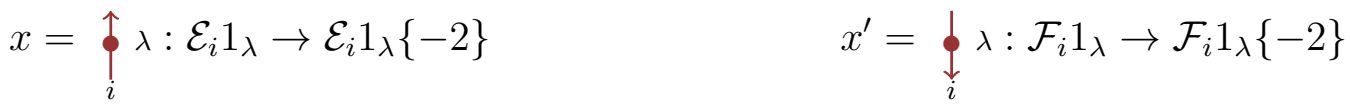

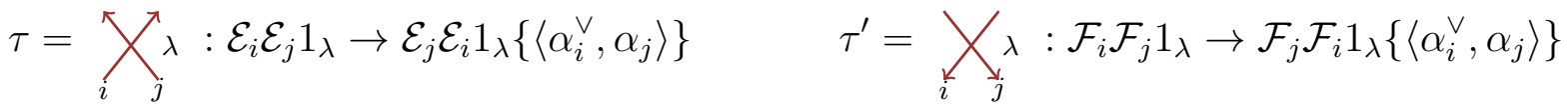

$$
\begin{aligned}
& \eta=\bigcup_{\lambda}^{\hat{\gamma}}: 1_{\lambda} \rightarrow \mathcal{F}_{i} \mathcal{E}_{i} 1_{\lambda}\left\{1-\left\langle{ }^{\theta} \alpha_{i}^{\vee}, \lambda+\alpha_{i}\right\rangle\right\} \quad \eta^{\prime}=\bigcup_{\lambda}^{i}: 1_{\lambda} \rightarrow \mathcal{E}_{i} \mathcal{F}_{i} 1_{\lambda}\left\{-1+\left\langle{ }^{\theta} \alpha_{i}^{\vee}, \lambda\right\rangle\right\} \\
& \epsilon=\bigcap_{i}^{\lambda}: \mathcal{E}_{i} \mathcal{F}_{i} 1_{\lambda} \rightarrow 1_{\lambda}\left\{-1+\left\langle{ }^{\theta} \alpha_{i}^{\vee}, \lambda\right\rangle\right\} \quad \epsilon^{\prime}=\bigcap_{i}^{\lambda}: \mathcal{F}_{i} \mathcal{E}_{i} 1_{\lambda} \rightarrow 1_{\lambda}\left\{1-\left\langle{ }^{\theta} \alpha_{i}^{\vee}, \lambda+\alpha_{i}\right\rangle\right\} .
\end{aligned}
$$

The 2-morphisms are subject to the following relations (1)-(7):

(1) (Adjunction)

$$
\bigcap_{i} \uparrow_{\lambda}=\uparrow_{i}=\uparrow \bigcap_{\lambda}, \quad \quad \bigcup_{i} \bigcap_{\lambda}=l^{i} \lambda=\bigcap \bigcup^{i} \lambda .
$$


(2) (Cyclicity of $x$ and $\tau$ )

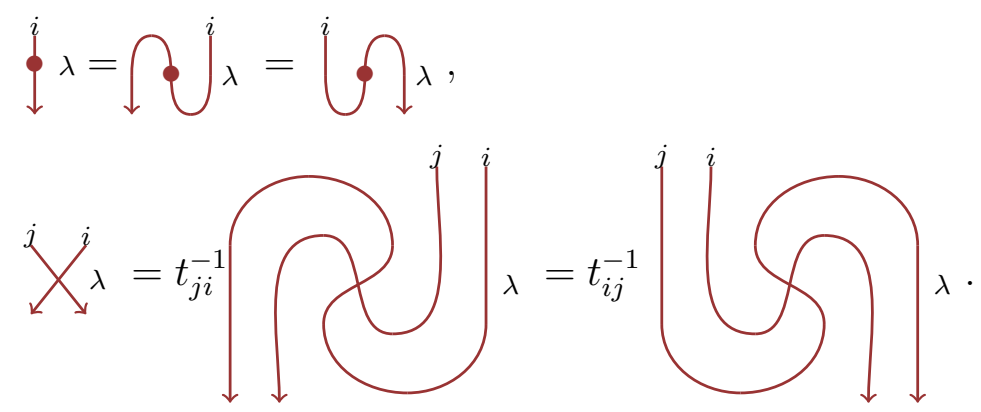

(3) (Quiver Hecke relations)

$$
\begin{aligned}
& Y_{i}^{\lambda}-X_{i}^{\lambda}=\sum_{i}^{\lambda}-\sum_{i}^{\lambda}= \begin{cases}\uparrow_{i}^{\uparrow} \uparrow_{j} & \text { if } i=j \\
0 & \text { otherwise }\end{cases} \\
& \bigotimes_{i}^{\lambda}= \begin{cases}0 & \text { if } i=j, \\
\uparrow_{i} \uparrow_{\lambda} & \text { if }|i-j|>1 \\
t_{i j} \uparrow_{i}^{\uparrow} \uparrow_{j}+t_{j i} \uparrow_{i}^{\uparrow} \lambda & \text { otherwise, }\end{cases} \\
& \searrow_{i}-\sum_{i}^{\lambda}= \begin{cases}t_{i j} \uparrow_{i} \uparrow_{j} \uparrow_{k} & \text { if } i=k \neq j \text { and }|i-j|=1, \\
0 & \text { otherwise. }\end{cases}
\end{aligned}
$$

(4) (Bubble relations)

$$
\begin{aligned}
& s \bigcup_{i}^{\lambda} \lambda=0 \text { if } s<\left\langle{ }^{\theta} \alpha_{i}^{\vee}, \lambda\right\rangle-1, \quad s \bigcup_{i}^{\dagger} \lambda=2^{\delta_{i, \diamond}} 1_{1_{\lambda}} \text { if } s=\left\langle{ }^{\theta} \alpha_{i}^{\vee}, \lambda\right\rangle-1 \text {, } \\
& { }_{\lambda} \bigcirc_{i}^{s}=0 \text { if } s<1-\left\langle{ }^{\theta} \alpha_{i}^{\vee}, \lambda+\alpha_{i}\right\rangle, \quad \quad{ }_{i} s=1_{1_{\lambda}} \text { if } s=1-\left\langle{ }^{\theta} \alpha_{i}^{\vee}, \lambda+\alpha_{i}\right\rangle \text {, }
\end{aligned}
$$

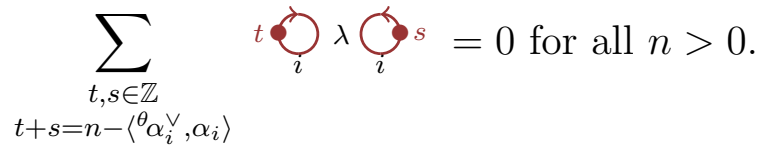

(5) (Nodal relations) Set

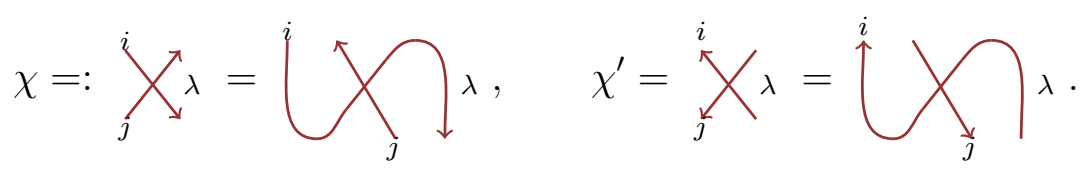


Then for any $i$ we have

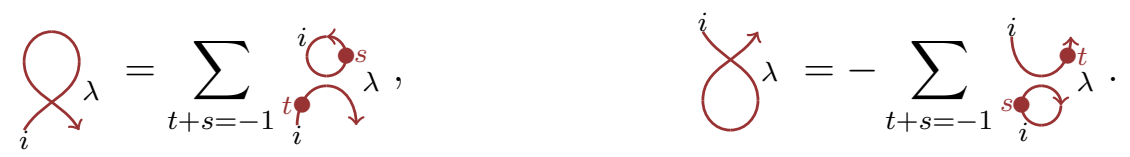

(6) (Bicross relations)

For $i \neq \diamond$, we have

$$
\begin{aligned}
& \bigotimes_{i}=\sum_{u+s+t=-2} \underbrace{i \bigcap_{i}^{\lambda}}_{i}-\left.\uparrow_{i}^{i}\right|_{i} \lambda, \\
& \bigodot_{i}^{\lambda} \lambda=\sum_{u+s+t=-2} \underbrace{i}_{\bigcap_{i}^{i} i}-\downarrow_{i} \uparrow_{i} \lambda .
\end{aligned}
$$

For $i=\diamond$, we have

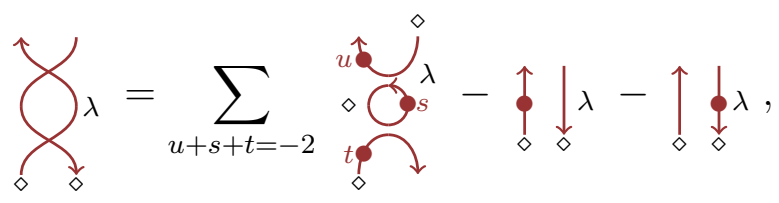

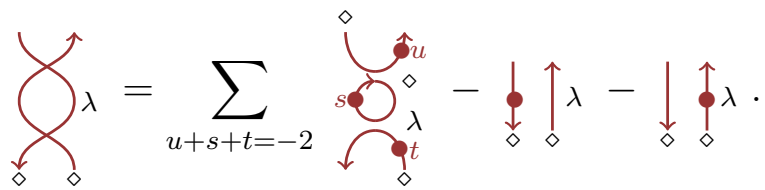

In all the sums above, the indices that are not on a circle run over non-negative integers. (7) (Mixed relations) For any $i \neq j$,

$$
\bigotimes_{i}^{\lambda} \lambda=\left.\uparrow_{i}\right|_{j} \lambda
$$$$
\bigotimes_{i} \lambda=\downarrow_{i} \uparrow_{j} \lambda
$$

Remark 3.2. The bubbles with negative labels are determined by the relation (3.9) and called fake bubbles. Note that because of (3.7), these can only be defined when 2 is invertible.

We organize the generating 2-morphisms with their degrees (other than the identity 2morphisms of degree 0 and $x, x^{\prime}$ of degree 2 ) in the following table.

\begin{tabular}{|c|c|c|c|c|c|c|}
\hline & $\bigcup_{\lambda}$ & $\Upsilon_{\lambda}^{i}$ & $\bigcap_{i}^{\lambda}$ & $\bigcap_{i}^{\lambda}$ & $\chi_{i}^{\lambda}$ & $\chi_{i}{ }_{j}^{\lambda}$ \\
\hline $\operatorname{deg}$ & $\left\langle{ }^{\theta} \alpha_{i}^{\vee}, \lambda+\alpha_{i}\right\rangle-1$ & $1-\left\langle{ }^{\theta} \alpha_{i}^{\vee}, \lambda\right\rangle$ & $1-\left\langle{ }^{\theta} \alpha_{i}^{\vee}, \lambda\right\rangle$ & $\left\langle{ }^{\theta} \alpha_{i}^{\vee}, \lambda+\alpha_{i}\right\rangle-1$ & $-\left\langle\alpha_{i}^{\vee}, \alpha_{j}\right\rangle$ & $-\left\langle\alpha_{i}^{\vee}, \alpha_{j}\right\rangle$ \\
\hline
\end{tabular}


The grading on $\widetilde{\mathfrak{U}}^{j}$ is well defined, i.e., all the relations in the definition are homogeneous. This can be checked in the same way as in [KL10]. Note that the main difference here is that

$$
-1+\left\langle{ }^{\theta} \alpha_{i}^{\vee}, \lambda+\alpha_{i}\right\rangle= \begin{cases}1+\lambda_{i}, & \text { if } i \neq \diamond, \\ 2+\lambda_{i}, & \text { if } i=\diamond .\end{cases}
$$

It follows that the diagrams $\chi, \chi^{\prime}$ in (3.10) have degree zero unless $i=j=\diamond$, and in that case they both have degree 1.

Note that subcategory of $\widetilde{\mathfrak{U}}^{j}$ generated by $\mathcal{E}_{i}, \mathcal{F}_{i}$ with $i>\diamond$ satisfy the relations of KhovanovLauda's 2-category $\mathfrak{U}_{r-1}$ of rank $r-1$. This yields a functor $\mathfrak{U}_{r-1} \rightarrow \widetilde{\mathfrak{U}}^{\jmath}$. We will show in a future work that this functor is faithful.

3.2. The coideal 2-category $\mathfrak{U}^{\mathfrak{J}}$. For the sake of simplicity, we make the following convention. We will omit the index $\diamond$ from the diagrams, that is, all the strands without labels should be viewed as labeled by $\diamond$.

Definition 3.3. The coideal 2-category $\mathfrak{U}^{\jmath}$ is the quotient of the 2-category $\widetilde{\mathfrak{U}}^{\jmath}$ by the relation

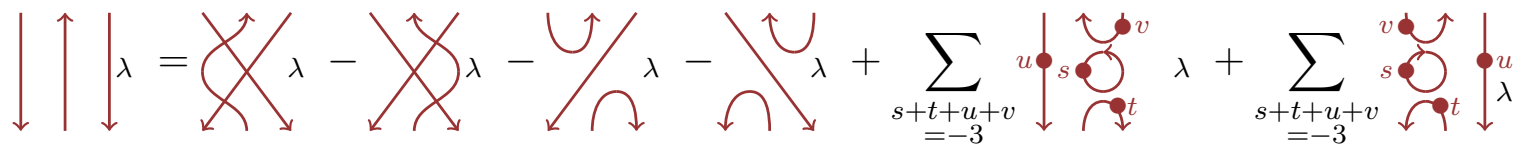

This 2-category $\mathfrak{U}^{\mathfrak{J}}$ admits an induced grading from $\widetilde{\mathfrak{U}}^{\mathfrak{l}}$ as the relation (3.17) is homogeneous of degree 0 .

We let $\mathfrak{U}^{j}$ denote the idempotent completion of $\mathfrak{U}^{\mathfrak{J}}$. Note that since the quiver Hecke relation holds in $\mathfrak{U}^{\jmath}$, the divided powers $\mathcal{E}_{i}^{(a)}, \mathcal{F}_{i}^{(a)}$ are well defined in $\dot{\mathfrak{U}}^{j}$, see for example KL10, Section 3.5]. We may also write $\mathcal{E}_{-i}=\mathcal{F}_{i}$ for $i \in \mathbb{I}^{\jmath}$ whenever this is convenient.

Remark 3.4. Note that we have automatically the relation

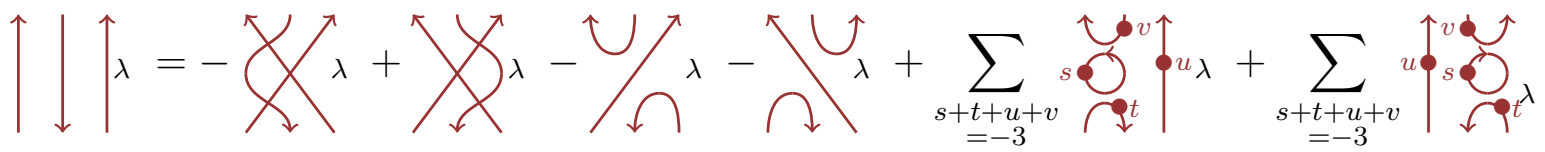

in $\mathfrak{U}^{\mathfrak{J}}$, since it is the image of (3.17) under the isomorphism $\operatorname{End}\left(\mathcal{F}_{\diamond} \mathcal{E}_{\diamond} \mathcal{F}_{\diamond}\right) \cong \operatorname{End}\left(\mathcal{E}_{\diamond} \mathcal{F}_{\diamond} \mathcal{E}_{\diamond}\right)$ given by adjunction.

3.3. Symmetries of $\mathfrak{U}^{\mathfrak{J}}$. Recall [KL10, 3.3.3] that the KLR 2-category $\mathfrak{U}$ admits several symmetries which categorify the standard (anti-)involutions for the quantum groups. We will describe the analogues of such symmetries for $\mathfrak{U}^{\mathfrak{J}}$, which categorify the three (anti-)involutions in Lemma 2.1. We will use the notation of [KL10, 3.3.3] and let $\left(\mathfrak{U}^{\jmath}\right)^{\mathrm{op}},\left(\mathfrak{U}^{\jmath}\right)^{\mathrm{co}},\left(\mathfrak{U}^{\mathrm{J}}\right)^{\mathrm{coop}}$ to denote the 2-category $\mathfrak{U}^{\mathfrak{l}}$ with the composition of 1-morphisms, 2-morphisms and both 1- and 2-morphisms reversed, respectively.

\section{Definition 3.5.}


(a) Consider the functor

$$
\psi_{\jmath}: \mathfrak{U}^{\mathfrak{J}} \longrightarrow\left(\mathfrak{U}^{\mathfrak{J}}\right)^{\mathrm{co}}
$$

which sends

$$
\lambda \mapsto \lambda, \quad 1_{\mu} \mathcal{E}_{i_{1}} \cdots \mathcal{E}_{i_{\ell}} 1_{\lambda}\{t\} \mapsto 1_{\mu} \mathcal{E}_{i_{1}} \cdots \mathcal{E}_{i_{\ell}} 1_{\lambda}\{-t\},
$$

for $\lambda, \mu \in \mathrm{X}_{\jmath}, t \in \mathbb{Z}$, and $i_{1}, \ldots, i_{\ell} \in \mathbb{I}^{\jmath}$. It acts on 2-morphisms by reflection through the $x$-axis and reversing orientation of all strands. Note that this action on 2-morphisms preserves all defining relations on 2-morphisms in $\mathfrak{U}^{\mathfrak{l}}$. It induces a duality on $\dot{\mathfrak{U}}^{\mathfrak{l}}$ which will be again denoted by $\psi_{\jmath}$. We have $\psi_{\jmath}^{2}=\mathrm{id}$ and in particular $\psi_{\jmath}$ is invertible.

(b) Consider the functor

$$
\omega_{\jmath}: \mathfrak{U}^{\mathfrak{l}} \longrightarrow \mathfrak{U}^{\mathfrak{l}}
$$

which sends (recall we write $\mathcal{E}_{-i}=\mathcal{F}_{i}$ for $i \in \mathbb{I}^{j}$ )

$$
\lambda \mapsto-\lambda-\varpi, \quad 1_{\mu} \mathcal{E}_{i_{1}} \cdots \mathcal{E}_{i_{\ell}} 1_{\lambda}\{t\} \mapsto 1_{-\lambda-\varpi} \mathcal{E}_{-i_{1}} \cdots \mathcal{E}_{-i_{\ell}} 1_{-\mu-\varpi}\{t\} .
$$

It acts on 2-morphisms by

$$
\begin{aligned}
& x \mapsto x^{\prime}, \quad x^{\prime} \mapsto x \quad \tau \mapsto-\tau^{\prime}, \quad \tau^{\prime} \mapsto-\tau, \\
& \eta \mapsto 2^{-\delta_{\diamond, i}} \eta^{\prime}, \quad \quad \eta^{\prime} \mapsto \eta, \quad \epsilon \mapsto 2^{\delta_{\diamond, i}} \epsilon^{\prime}, \quad \epsilon^{\prime} \mapsto \epsilon .
\end{aligned}
$$

Thus, each diagram is sent to the diagram with reversed orientation, times certain unit in $\mathbf{k}$. The functor $\omega_{j}$ is an equivalence. An inverse is provided by the functor which coincides with $\omega_{\text {j }}$ on objects and 1-morphisms, and acts on 2-morphisms

$$
\begin{aligned}
& x \mapsto x^{\prime}, \quad x^{\prime} \mapsto x \quad \tau \mapsto-\tau^{\prime}, \quad \tau^{\prime} \mapsto-\tau, \\
& \eta \mapsto \eta^{\prime}, \quad \eta^{\prime} \mapsto 2^{\delta_{\diamond, i}} \eta, \quad \epsilon \mapsto \epsilon^{\prime}, \quad \epsilon^{\prime} \mapsto 2^{-\delta_{\diamond, i} \epsilon .}
\end{aligned}
$$

(c) Consider the functor

$$
\sigma_{\jmath}: \mathfrak{U}^{\mathfrak{l}} \longrightarrow\left(\mathfrak{U}^{\mathfrak{J}}\right)^{\mathrm{op}}
$$

which sends

$$
\lambda \mapsto-\lambda-\varpi, \quad 1_{\mu} \mathcal{E}_{i_{1}} \cdots \mathcal{E}_{i_{\ell}} 1_{\lambda}\{t\} \mapsto 1_{-\lambda-\varpi} \mathcal{E}_{i_{\ell}} \cdots \mathcal{E}_{i_{1}} 1_{-\mu-\varpi}\{t\} .
$$

It acts on 2-morphisms by

$$
\begin{aligned}
& x \mapsto x, \quad x^{\prime} \mapsto x^{\prime} \quad \tau \mapsto-\tau, \quad \tau^{\prime} \mapsto-\tau^{\prime}, \\
& \eta \mapsto 2^{-\delta_{i, \diamond}} \eta^{\prime}, \quad \quad \eta^{\prime} \mapsto \eta, \quad \epsilon \mapsto 2^{\delta_{i, \diamond}} \epsilon^{\prime} \quad \epsilon^{\prime} \mapsto \epsilon
\end{aligned}
$$

Thus, every diagram is sent to its reflection through the $y$-axis, times certain unit in $\mathbf{k}$. The functor $\sigma_{j}$ is an equivalence. An inverse is provided by the functor which coincides with $\sigma_{\jmath}$ on objects and 1-morphisms and acts on 2-morphisms as

$$
\begin{aligned}
& x \mapsto x, \quad x^{\prime} \mapsto x^{\prime} \quad \tau \mapsto-\tau, \quad \tau^{\prime} \mapsto-\tau^{\prime}, \\
& \eta \mapsto \eta^{\prime}, \quad \quad \eta^{\prime} \mapsto 2^{\delta_{i, \diamond}} \eta, \quad \epsilon \mapsto \epsilon^{\prime} \quad \quad \epsilon^{\prime} \mapsto 2^{-\delta_{i, \diamond}} \epsilon .
\end{aligned}
$$

Remark 3.6. The presence of the factors of 2 above reflects an asymmetry in the definition of $\mathfrak{U}^{\prime}$, specifically in the relations (3.7)-(3.8). We can rescale the generators $\eta^{\prime}$ and $\epsilon^{\prime}$ so that the bubbles in (3.7)-(3.8) evaluate to any pair of scalars whose product is $2^{\delta_{i, \diamond}}$. One obvious choice is to switch the role of clockwise and counterclockwise bubbles; we denote the 
resulting (isomorphic) 2-category with this presentation ' $\mathfrak{U}^{3}$. Alternatively, we could give a more symmetric definition by choosing a square root $\sqrt{2}$, and using $\sqrt{2}^{\delta_{i, \diamond}}$ in both (3.7) and (3.8). In this case, the definitions of $\sigma_{3}$ and $\omega_{\text {, }}$ would not require the factors of 2 .

One perspective on how these different forms arise is that this 2-category is connected to geometry and representation theory in types B and C. Many aspects of these constructions, such as the cohomology rings of the partial flag varieties and the category of Soergel bimodules only depend on the Weyl group and its reflection representation, and thus are identical in types B/C. Others, such as the Demazure operators discussed in Section 5.3, and thus the formulas for integration on flag varieties do depend on the root systems, and thus differ by powers of 2 between types $\mathrm{B}$ and $\mathrm{C}$. The category $\mathfrak{U}^{\jmath}$ naturally arises from the computations with type B flag varieties (which we choose to use), while ' $\mathfrak{U}^{\jmath}$ would arise most naturally with type C geometry.

\section{The Grothendieck Group of $\mathfrak{U}^{3}$}

Throughout the rest of the paper, we assume that $\mathbf{k}$ is a complete local ring and continue to assume that 2 is a unit; equivalently, we require that the residue field of $\mathbf{k}$ is not of characteristic 2. A particularly important case is when $\mathbf{k}$ is itself a field of characteristic $\neq 2$.

In this section, we provide a categorification of the $\mathrm{J}$-Serre relations in the framework of 2 -category $\dot{\mathfrak{U}}^{j}$. This allows us to define a surjective map to the Grothendieck group of $\dot{\mathfrak{U}}^{j}$ from ${ }_{\mathcal{A}} \mathbf{U}^{\jmath}$.

4.1. Reformulation of $\jmath$-Serre relations. First let us rewrite the $\jmath$-Serre relation (2.10) in the form (4.1)-(4.3) and the $\mathrm{j}$-Serre relation (2.9) in the form (4.4)-(4.6), respectively:

$$
\begin{array}{lr}
\mathcal{F}_{\diamond} \mathcal{E}_{\diamond} \mathcal{F}_{\diamond} 1_{\lambda}=\mathcal{F}_{\diamond}^{(2)} \mathcal{E}_{\diamond} 1_{\lambda}+\left(\mathcal{E}_{\diamond} \mathcal{F}_{\diamond}^{(2)}-\left[\lambda_{\diamond}-2\right] \mathcal{F}\right) 1_{\lambda}+\left[\lambda_{\diamond}\right] \cdot \mathcal{F}_{\diamond} 1_{\lambda}, & \text { if } \lambda_{\diamond} \geq 2 ; \\
\mathcal{F}_{\diamond} \mathcal{E}_{\diamond} \mathcal{F}_{\diamond} 1_{\lambda}=\mathcal{F}_{\diamond}^{(2)} \mathcal{E}_{\diamond} 1_{\lambda}+\mathcal{E}_{\diamond} \mathcal{F}_{\diamond}^{(2)} 1_{\lambda}+2 \cdot \mathcal{F}_{\diamond} 1_{\lambda}, & \text { if } \lambda_{\diamond}=1 ; \\
\mathcal{F}_{\diamond} \mathcal{E}_{\diamond} \mathcal{F}_{\diamond} 1_{\lambda}=\mathcal{E}_{\diamond} \mathcal{F}_{\diamond}^{(2)} 1_{\lambda}+\left(\mathcal{F}_{\diamond}^{(2)} \mathcal{E}_{\diamond}-\left[-\lambda_{\diamond}\right] \mathcal{F}\right) 1_{\lambda}+\left[-\lambda_{\diamond}+2\right] \cdot \mathcal{F}_{\diamond} 1_{\lambda}, & \text { if } \lambda_{\diamond} \leq 0 ; \\
\mathcal{E}_{\diamond} \mathcal{F}_{\diamond} \mathcal{E}_{\diamond} 1_{\lambda}=\mathcal{E}_{\diamond}^{(2)} \mathcal{F}_{\diamond} 1_{\lambda}+\left(\mathcal{F}_{\diamond} \mathcal{E}_{\diamond}^{(2)}-\left[-3-\lambda_{\diamond}\right] \mathcal{E}_{\diamond}\right) 1_{\lambda}+\left[-1-\lambda_{\diamond}\right] \cdot \mathcal{E}_{\diamond} 1_{\lambda}, & \text { if } \lambda_{\diamond} \leq-3 ; \\
\mathcal{E}_{\diamond} \mathcal{F}_{\diamond} \mathcal{E}_{\diamond} 1_{\lambda}=\mathcal{F}_{\diamond} \mathcal{E}_{\diamond}^{(2)} 1_{\lambda}+\mathcal{E}_{\diamond}^{(2)} \mathcal{F}_{\diamond} 1_{\lambda}+2 \cdot \mathcal{E}_{\diamond} 1_{\lambda}, & \text { if } \lambda_{\diamond}=-2 ; \\
\mathcal{E}_{\diamond} \mathcal{F}_{\diamond} \mathcal{E}_{\diamond} 1_{\lambda}=\mathcal{F}_{\diamond} \mathcal{E}_{\diamond}^{(2)} 1_{\lambda}+\left(\mathcal{E}_{\diamond}^{(2)} \mathcal{F}_{\diamond}-\left[1+\lambda_{\diamond}\right] \mathcal{E}_{\diamond}\right) 1_{\lambda}+\left[\lambda_{\diamond}+3\right] \cdot \mathcal{E}_{\diamond} 1_{\lambda}, & \text { if } \lambda_{\diamond} \geq-1 .
\end{array}
$$

In each of the formulas (4.1) -(4.6) above the right-hand side is the expansion of the left hand side in terms of canonical basis elements of ${ }_{\mathcal{A}} \mathbf{U}^{3}$. Knowing these expansions facilitates understanding the structure of the corresponding 1-morphisms in $\mathfrak{U}^{3}$, but we will not use explicitly the fact that this is the canonical basis expansion. It can be proved in several ways, but we will leave aside the proof. In fact, we can also derive this from Theorem 6.5 below by showing that the factors on the right-hand sides correspond to indecomposable self-dual 1-morphisms in the 2-category $\dot{\mathfrak{U}}^{\jmath}$ (see Remark 4.2 below).

4.2. Categorification of $\jmath$-Serre (4.1). We denote by $\mathcal{E}_{\diamond} \mathcal{F}_{\diamond}^{(2)} 1_{\lambda}$ the idempotent $\uparrow \searrow^{\lambda}$ in $\operatorname{End}\left(\mathcal{E}_{\diamond} \mathcal{F}_{\diamond}^{2} 1_{\lambda}\right)$ as a 1 -morphism in $\dot{\mathfrak{U}}$. 
Lemma 4.1. Let $\lambda \in \mathrm{X}_{\text {J }}$ such that $\lambda_{\diamond}=\left\langle{ }^{\theta} \alpha_{\diamond}^{\vee}, \lambda\right\rangle \geq 2$.

(a) The element

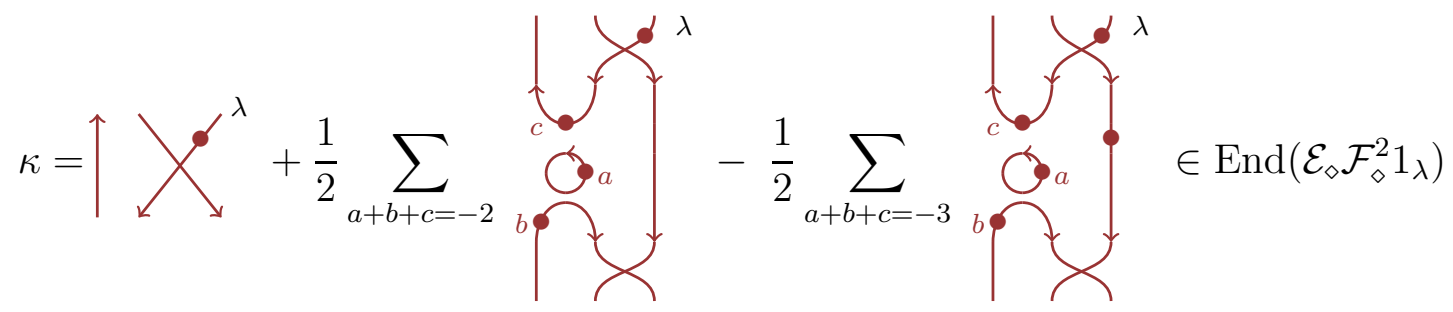

is an idempotent. We denote the image of this element in $\mathcal{E}_{\diamond} \mathcal{F}_{\diamond}^{2}$ by $\left(\mathcal{E}_{\diamond} \mathcal{F}_{\diamond}^{(2)}-\left[\lambda_{\diamond}-2\right] \mathcal{F}_{\diamond}\right) 1_{\lambda}$ when considered as a 1-morphism in the idempotent completion of $\widetilde{\mathfrak{U}}^{\jmath}$.

(b) We have the following isomorphism of 1-morphisms in the idempotent completion of $\widetilde{\mathfrak{U}}^{3}$,

$$
\mathcal{E}_{\diamond} \mathcal{F}_{\diamond}^{(2)} 1_{\lambda} \cong\left(\mathcal{E}_{\diamond} \mathcal{F}_{\diamond}^{(2)}-\left[\lambda_{\diamond}-2\right] \mathcal{F}_{\diamond}\right) 1_{\lambda} \oplus \mathcal{F}_{\diamond} 1_{\lambda}^{\oplus\left[\lambda_{\diamond}-2\right]}
$$

Proof. We shall prove the two statements together. We first have the following system of 2-morphisms

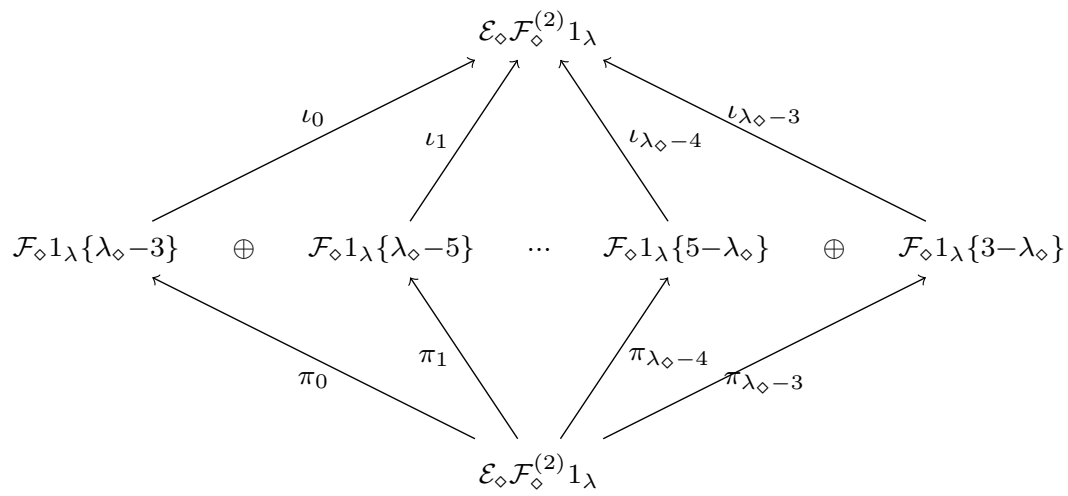

where the 2-morphisms $\iota_{s}$ and $\pi_{s}$ are defined as follows $\left(0 \leq s \leq \lambda_{\diamond}-3\right)$

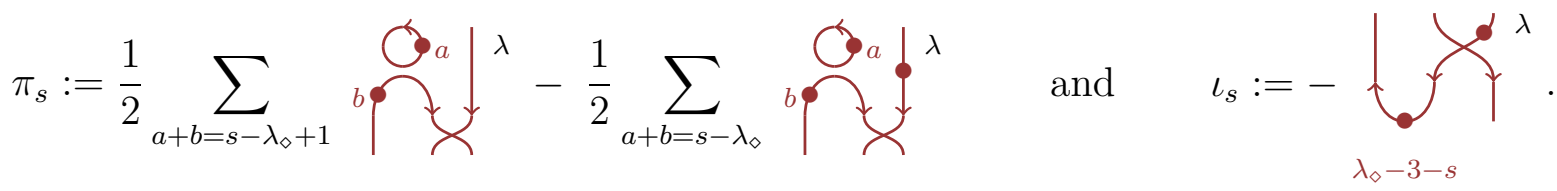

We have $\left(0 \leq s, s^{\prime} \leq \lambda_{\diamond}-3\right)$

$$
\pi_{s} \cdot \iota_{s^{\prime}}=-\frac{1}{2} \sum_{a+b=s-\lambda_{\diamond}+1}+\frac{1}{2} \sum_{a+b=s-\lambda_{\diamond}}^{\lambda}
$$


Then we have

$$
\begin{aligned}
& -\left.\frac{1}{2} \sum_{a+b=s-\lambda_{\diamond}+1} \bigcirc_{a}^{\lambda}\right|_{c} ^{\lambda}= \begin{cases}\downarrow^{\downarrow} s^{\prime}, & \text { if } s-s^{\prime} \geq 0 ; \\
0, & \text { if } s-s^{\prime}<0 .\end{cases}
\end{aligned}
$$

Therefore $\pi_{s} \cdot \iota_{s^{\prime}}=\delta_{s, s^{\prime}}$. Hence we know $\iota_{s} \pi_{s}\left(0 \leq s \leq \lambda_{\diamond}-3\right)$ are mutually orthogonal idempotents in $\mathcal{U}^{\jmath}\left(\mathcal{E}_{\diamond} \mathcal{F}_{\diamond}^{2} 1_{\lambda}, \mathcal{E}_{\diamond} \mathcal{F}_{\diamond}^{2} 1_{\lambda}\right)$. The idempotent

$$
\kappa=\mathrm{id}_{\mathcal{E}_{\diamond} \mathcal{F}_{\diamond}^{(2)} 1_{\lambda}}-\sum_{0 \leq s \leq \lambda_{\diamond}-3} \iota_{s} \pi_{s}
$$

can then be expressed diagrammatically as in the lemma.

Remark 4.2. One can show that $\left(\mathcal{E}_{\diamond} \mathcal{F}_{\diamond}^{(2)}-\left[\lambda_{\diamond}-2\right] \mathcal{F}_{\diamond}\right) 1_{\lambda}$ is indecomposable in $\dot{\mathfrak{U}}$. In fact, since $\pi_{t} \kappa=\kappa \iota_{s}=0$, a direct diagrammatic computation implies that the algebra $\operatorname{End}\left(\mathcal{E}_{\diamond} \mathcal{F}_{\diamond}^{(2)} 1_{\lambda}\right)$ is spanned by $\kappa$ and $\iota_{s+t} x^{s} \pi_{t}$ for $0 \leq s \leq s+t \leq \lambda_{\diamond}-3$. In particular, the algebra $\kappa \operatorname{End}\left(\mathcal{E}_{\diamond} \mathcal{F}_{\diamond}^{(2)} 1_{\lambda}\right) \kappa$ is a rank one $\mathbf{k}$-module spanned by $\kappa$. It is a local ring, hence $\left(\mathcal{E}_{\diamond} \mathcal{F}_{\diamond}^{(2)}-\left[\lambda_{\diamond}-2\right] \mathcal{F}_{\diamond}\right) 1_{\lambda}$ is indecomposable. Similar arguments can be used to show that all the individual terms appearing in the sums on the right-hand sides of (4.1)-(4.6) correspond to indecomposable 1-morphisms.

We denote by $\mathcal{F}_{\diamond}^{(2)} \mathcal{E}_{\diamond} 1_{\lambda}$ the 1 -morphism in the idempotent completion of $\tilde{\mathfrak{U}}^{\jmath}$ (or in $\dot{\mathfrak{U}^{j}}$ ) defined as the image of the idempotent $\searrow \uparrow^{\lambda} \in \operatorname{End}\left(\mathcal{F}_{\diamond}^{2} \mathcal{E}_{\diamond} 1_{\lambda}\right)$.

Proposition 4.3. Let $\lambda \in \mathrm{X}_{\jmath}$ with $\lambda_{\diamond}=\left\langle{ }^{\theta} \alpha_{\diamond}^{\vee}, \lambda\right\rangle \geq 2$. Then,

(a) we have the following split surjection in the idempotent completion of $\tilde{\mathfrak{U}}^{3}$ (and in $\dot{\mathfrak{U}}^{j}$ ),

$$
\mathcal{F}_{\diamond}^{(2)} \mathcal{E}_{\diamond} 1_{\lambda} \oplus\left(\mathcal{E}_{\diamond} \mathcal{F}_{\diamond}^{(2)}-\left[\lambda_{\diamond}-2\right] \mathcal{F}_{\diamond}\right) 1_{\lambda} \oplus \mathcal{F}_{\diamond} 1_{\lambda}^{\oplus\left[\lambda_{\diamond}\right]} \longrightarrow \mathcal{F}_{\diamond} \mathcal{E}_{\diamond} \mathcal{F}_{\diamond} 1_{\lambda}
$$

(b) we have the following isomorphism in $\dot{\mathfrak{U}}^{3}$

$$
\mathcal{F}_{\diamond} \mathcal{E}_{\diamond} \mathcal{F}_{\diamond} 1_{\lambda} \cong \mathcal{F}_{\diamond}^{(2)} \mathcal{E}_{\diamond} 1_{\lambda} \oplus\left(\mathcal{E}_{\diamond} \mathcal{F}_{\diamond}^{(2)}-\left[\lambda_{\diamond}-2\right] \mathcal{F}_{\diamond}\right) 1_{\lambda} \oplus \mathcal{F}_{\diamond} 1_{\lambda}^{\oplus\left[\lambda_{\diamond}\right]}
$$

Proof. We introduce the following diagrams $\left(0 \leq k \leq \lambda_{\diamond}-1\right)$ :

$$
\begin{aligned}
& B_{1}=-\chi^{\lambda}, \quad B_{2}=\chi^{\lambda}, \quad P_{k}=\downarrow_{\overbrace{}^{\lambda}}^{\lambda_{\diamond}-1-k}, \\
& C_{1}=\chi^{\lambda}, \quad C_{2}=\chi^{\lambda}, \quad I_{k}=\frac{1}{2} \sum_{s+t=k} \bigcup_{-\lambda_{\diamond}-2+t}^{\bigcup_{s}} .
\end{aligned}
$$


Following (A.8)-(A.9), we introduce modifications of $I_{\lambda_{\diamond}-1}$ and $P_{0}$ as follows:

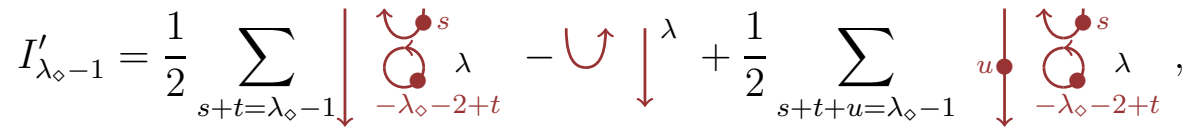

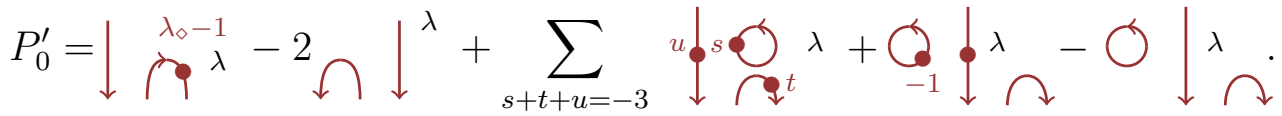

Recall the 2-morphism $\kappa \in \operatorname{End}\left(\mathcal{E}_{\diamond} \mathcal{F}_{\diamond}^{2} 1_{\lambda}\right)$ in Lemma 4.1. We define the 2-morphisms:

$$
\begin{gathered}
{\left[\begin{array}{c}
B_{1} \\
\kappa \cdot B_{2} \\
P_{0}^{\prime} \\
P_{1} \\
\vdots \\
P_{\lambda_{\diamond}-1}
\end{array}\right]: \mathcal{F}_{\diamond} \mathcal{E}_{\diamond} \mathcal{F}_{\diamond} 1_{\lambda} \longrightarrow \mathcal{F}_{\diamond}^{(2)} \mathcal{E}_{\diamond} 1_{\lambda} \oplus\left(\mathcal{E}_{\diamond} \mathcal{F}_{\diamond}^{(2)}-\left[\lambda_{\diamond}-2\right] \mathcal{F}_{\diamond}\right) 1_{\lambda} \oplus \mathcal{F}_{\diamond} 1_{\lambda}^{\oplus\left[\lambda_{\diamond}\right]},} \\
{\left[\begin{array}{lllll}
C_{1} & C_{2} \cdot \kappa & I_{0} & \cdots & I_{\lambda_{\diamond}-1}^{\prime}
\end{array}\right]: \mathcal{F}_{\diamond}^{(2)} \mathcal{E}_{\diamond} 1_{\lambda} \oplus\left(\mathcal{E}_{\diamond} \mathcal{F}_{\diamond}^{(2)}-\left[\lambda_{\diamond}-2\right] \mathcal{F}_{\diamond}\right) 1_{\lambda} \oplus \mathcal{F}_{\diamond} 1_{\lambda}^{\oplus\left[\lambda_{\diamond}\right]} \longrightarrow \mathcal{F}_{\diamond} \mathcal{E}_{\diamond} \mathcal{F}_{\diamond} 1_{\lambda} .}
\end{gathered}
$$

The proof of the proposition is reduced to verifying the identities (4.11)-(4.12) below:

$$
\left[\begin{array}{c}
B_{1} \\
\kappa \cdot B_{2} \\
P_{0}^{\prime} \\
P_{1} \\
\vdots \\
P_{\lambda_{\diamond}-1}
\end{array}\right] \cdot\left[\begin{array}{llllll}
C_{1} & C_{2} \cdot \kappa & I_{0} & \cdots & I_{\lambda_{\diamond}-2} & I_{\lambda_{\diamond}-1}^{\prime}
\end{array}\right]=\operatorname{id}_{\left(\lambda_{\diamond}+2\right) \times\left(\lambda_{\diamond}+2\right)} \quad \text { in } \tilde{\mathfrak{U}}^{\jmath}
$$

and

$$
\left[\begin{array}{lllll}
C_{1} & C_{2} \cdot \kappa & I_{0} & \cdots & I_{\lambda_{\diamond}-1}^{\prime}
\end{array}\right] \cdot\left[\begin{array}{c}
B_{1} \\
\kappa \cdot B_{2} \\
P_{0}^{\prime} \\
\vdots \\
P_{\lambda_{\diamond}-1}^{\prime}
\end{array}\right]=\operatorname{id}_{\mathcal{F}_{\diamond} \mathcal{E}_{\diamond} \mathcal{F}_{\diamond} 1_{\lambda}} \quad \text { in } \dot{\mathfrak{U}}
$$

The proofs of the identities (4.11) -(4.12) via elementary but lengthy diagrammatic computations are given in the Appendix $\mathrm{A}$ (see Proposition A.14 and Proposition A.22).

4.3. Categorification of $\jmath$-Serre (4.2). In this subsection we shall assume $\lambda \in \mathrm{X}_{\text {, with }}$ $\lambda_{\diamond}=\left\langle{ }^{\theta} \alpha_{\diamond}^{\vee}, \lambda\right\rangle=1$, and recall in this case the expression of the $\mathrm{\jmath}$-Serre relation in (4.2). We denote by $\mathcal{F}_{\diamond}^{(2)} \mathcal{E}_{\diamond} 1_{\lambda}$ the image of the idempotent $\searrow^{\lambda} \in \operatorname{End}\left(\mathcal{F}_{\diamond}^{2} \mathcal{E}_{\diamond} 1_{\lambda}\right)$ and by $\mathcal{E}_{\diamond} \mathcal{F}_{\diamond}^{(2)} 1_{\lambda}$ the image of the idempotent $\searrow^{\lambda} \in \operatorname{End}\left(\mathcal{F}_{\diamond}^{2} \mathcal{E}_{\diamond} 1_{\lambda}\right)$ in the idempotent completion of $\tilde{\mathfrak{U}}^{\jmath}$ (or $\left.\dot{\mathfrak{U}}^{\jmath}\right)$.

Proposition 4.4. Let $\lambda \in \mathrm{X}_{\jmath}$ with $\lambda_{\diamond}=\left\langle{ }^{\theta} \alpha_{\diamond}^{\vee}, \lambda\right\rangle=1$. Then, 
(a) we have the following split surjection in the idempotent completion of $\tilde{\mathfrak{U}}^{\jmath}$ (and in $\dot{\mathfrak{U}}^{\jmath}$ ):

$$
\mathcal{F}_{\diamond}^{(2)} \mathcal{E}_{\diamond} 1_{\lambda} \oplus \mathcal{E}_{\diamond} \mathcal{F}_{\diamond}^{(2)} 1_{\lambda} \oplus \mathcal{F}_{\diamond} 1_{\lambda} \oplus \mathcal{F}_{\diamond} 1_{\lambda} \longrightarrow \mathcal{F}_{\diamond} \mathcal{E}_{\diamond} \mathcal{F}_{\diamond} 1_{\lambda}
$$

(b) we have the following isomorphism in $\dot{\mathfrak{U}}^{3}$ :

$$
\mathcal{F}_{\diamond} \mathcal{E}_{\diamond} \mathcal{F}_{\diamond} 1_{\lambda} \cong \mathcal{F}_{\diamond}^{(2)} \mathcal{E}_{\diamond} 1_{\lambda} \oplus \mathcal{E}_{\diamond} \mathcal{F}_{\diamond}^{(2)} 1_{\lambda} \oplus \mathcal{F}_{\diamond} 1_{\lambda} \oplus \mathcal{F}_{\diamond} 1_{\lambda}
$$

Proof. In this case, the relation (3.17) simplifies to the form:

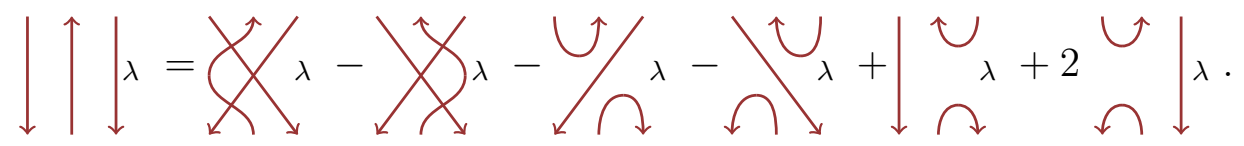

We introduce the following diagrams

$$
\begin{aligned}
& B_{1}=-\chi^{\lambda}, \quad B_{2}=\chi^{\lambda}, \quad P_{0}=\downarrow \bigcap^{\lambda}-\downarrow^{\lambda}, \quad P_{1}=\curvearrowleft \downarrow^{\lambda}, \\
& C_{1}=\searrow^{\lambda}, \quad C_{2}=\chi^{\lambda}, \quad I_{0}=\uparrow \downarrow^{\lambda}-\downarrow \bigcup^{\lambda}, \quad I_{1}=\bigcup \downarrow^{\lambda} .
\end{aligned}
$$

We consider the following 2-morphisms:

$$
\begin{aligned}
& {\left[\begin{array}{c}
B_{1} \\
B_{2} \\
P_{0} \\
P_{1}
\end{array}\right]: \mathcal{F}_{\diamond} \mathcal{E}_{\diamond} \mathcal{F}_{\diamond} 1_{\lambda} \longrightarrow \mathcal{F}_{\diamond}^{(2)} \mathcal{E}_{\diamond} 1_{\lambda} \oplus\left(\mathcal{E}_{\diamond} \mathcal{F}_{\diamond}^{(2)}-\left[\lambda_{\diamond}-2\right] \mathcal{F}_{\diamond}\right) 1_{\lambda} \oplus \mathcal{F}_{\diamond} 1_{\lambda}^{\oplus\left[\lambda_{\diamond}\right]},} \\
& {\left[\begin{array}{llll}
C_{1} & C_{2} & I_{0} & I_{1}
\end{array}\right]: \mathcal{F}_{\diamond}^{(2)} \mathcal{E}_{\diamond} 1_{\lambda} \oplus\left(\mathcal{E}_{\diamond} \mathcal{F}_{\diamond}^{(2)}-\left[\lambda_{\diamond}-2\right] \mathcal{F}_{\diamond}\right) 1_{\lambda} \oplus \mathcal{F}_{\diamond} 1_{\lambda}^{\oplus\left[\lambda_{\diamond}\right]} \longrightarrow \mathcal{F}_{\diamond} \mathcal{E}_{\diamond} \mathcal{F}_{\diamond} 1_{\lambda} \text {. }}
\end{aligned}
$$

The proof of the proposition is reduced to verifying the two identities

$$
\left[\begin{array}{l}
B_{1} \\
B_{2} \\
P_{0} \\
P_{1}
\end{array}\right] \cdot\left[\begin{array}{llll}
C_{1} & C_{2} & I_{0} & I_{1}
\end{array}\right]=\mathrm{id}_{4 \times 4} \quad \text { in } \widetilde{\mathfrak{U}}^{\jmath},
$$

and

$$
\left[\begin{array}{llll}
C_{1} & C_{2} & I_{0} & I_{1}
\end{array}\right] \cdot\left[\begin{array}{c}
B_{1} \\
B_{2} \\
P_{0} \\
P_{1}
\end{array}\right]=\operatorname{id}_{\mathcal{F}_{\diamond} \mathcal{E}_{\diamond} \mathcal{F}_{\diamond} 1_{\lambda}} \quad \text { in } \dot{\mathfrak{U}^{\jmath}} .
$$

The identities (4.14) and (4.15) admit proofs which are analogous (and much easier) to those of the identities (4.11) and (4.12) given in the Appendix A. We will leave the details of these computations to the reader. 
4.4. Categorification of $\mathrm{y}$-Serre (4.3)-(4.6). In this subsection, we complete the categorification of the $\mathrm{y}$-Serre relations (2.9) and (2.10) in the remaining cases (4.3)-(4.6) by using results from Sections 4.2 and 4.3 and the equivalences (3.18) and (3.19).

Indeed the categorification of the $\mathrm{y}$-Serre relation (4.1) implies the categorification of the $\mathrm{J}$ Serre relation (4.3) under the equivalence (3.19). Then entirely similarly the categorification of the $y$-Serre relations (4.1)-(4.3) implies the categorification of the $y$-Serre relations (4.4) - (4.6) under the equivalence (3.18). We shall only prove the categorification of the $\jmath$-Serre relation (4.3) here and leave the other cases to the reader.

Proposition 4.5. Let $\lambda \in X_{\jmath}$ with $\lambda_{\diamond}=\left\langle{ }^{\theta} \alpha_{\diamond}^{\vee}, \lambda\right\rangle \leq 0$.

(a) We have the following isomorphism of 1-morphisms in $\dot{\mathfrak{U}}^{3}$ :

$$
\mathcal{F}_{\diamond}^{(2)} \mathcal{E}_{\diamond} 1_{\lambda} \cong\left(\mathcal{F}_{\diamond}^{(2)} \mathcal{E}_{\diamond}-\left[-\lambda_{\diamond}\right] \mathcal{F}_{\diamond}\right) 1_{\lambda} \oplus \mathcal{F}_{\diamond} 1_{\lambda}^{\oplus\left[-\lambda_{\diamond}\right]}
$$

where $\left(\mathcal{F}_{\diamond}^{(2)} \mathcal{E}_{\diamond}-\left[-\lambda_{\diamond}\right] \mathcal{F}_{\diamond}\right) 1_{\lambda}$ is the image of the idempotent $\kappa$ in Lemma 4.1] under the equivalence $\sigma_{\jmath}$ in (3.19).

(b) We have the following split surjection in the idempotent completion of $\tilde{\mathfrak{U}}^{j}$ (and in $\dot{\mathfrak{U}}^{j}$ ):

$$
\mathcal{E}_{\diamond} \mathcal{F}_{\diamond}^{(2)} 1_{\lambda} \oplus\left(\mathcal{F}_{\diamond}^{(2)} \mathcal{E}_{\diamond}-\left[-\lambda_{\diamond}\right] \mathcal{F}_{\diamond}\right) 1_{\lambda} \oplus \mathcal{F}_{\diamond} 1_{\lambda}^{\oplus\left[-\lambda_{\diamond}+2\right]} \longrightarrow \mathcal{F}_{\diamond} \mathcal{E}_{\diamond} \mathcal{F}_{\diamond} 1_{\lambda}
$$

(c) We have the following isomorphism in $\dot{\mathfrak{U}}^{3}$ :

$$
\mathcal{F}_{\diamond} \mathcal{E}_{\diamond} \mathcal{F}_{\diamond} 1_{\lambda} \cong \mathcal{E}_{\diamond} \mathcal{F}_{\diamond}^{(2)} 1_{\lambda} \oplus\left(\mathcal{F}_{\diamond}^{(2)} \mathcal{E}_{\diamond}-\left[-\lambda_{\diamond}\right] \mathcal{F}_{\diamond}\right) 1_{\lambda} \oplus \mathcal{F}_{\diamond} 1_{\lambda}^{\oplus\left[-\lambda_{\diamond}+2\right]} .
$$

Proof. Let $\lambda^{\prime}=-\lambda+\alpha_{\diamond}-\varpi$. We have $\lambda_{\diamond}^{\prime}=-\lambda_{\diamond}+2 \geq 2$ by assumption. Thus, the desired maps are just the image under the equivalence $\sigma_{\jmath}$ of the split surjection and the isomorphism in Lemma 4.1 and Propositions 4.34 .5 applied to $\mathcal{F}_{\diamond} \mathcal{E}_{\diamond} \mathcal{F}_{\diamond} 1_{\lambda^{\prime}}$.

\subsection{The Grothendieck group.}

Lemma 4.6. The assignment $\lambda \mapsto \lambda, \mathcal{E}_{i}^{(a)} 1_{\lambda} \mapsto\left[\mathcal{E}_{i}^{(a)} 1_{\lambda}\right], \mathcal{F}_{i}^{(a)} 1_{\lambda} \mapsto\left[\mathcal{F}_{i}^{(a)} 1_{\lambda}\right]$ for all $\lambda \in \mathrm{X}_{\jmath}, i \in$ $\mathbb{I}^{\jmath}, a \in \mathbb{N}$ defines an $\mathcal{A}$-linear functor $\aleph: \dot{\mathcal{U}}^{\jmath} \rightarrow K_{0}(\dot{\mathfrak{U}})$. Furthermore, we have ${ }^{\prime} \circ \psi_{\jmath}=\psi_{\jmath} \circ \aleph$.

Proof. It suffices to prove that the assignment in the lemma defines a $\mathbb{Q}(q)$-linear functor $\dot{\mathbf{U}}^{\jmath} \rightarrow \mathbb{Q}(q) \otimes_{\mathcal{A}} K_{0}\left(\dot{\mathfrak{U}}^{\jmath}\right)$. To this end, we must check that all defining relations $(2.6)-(2.10)$ in $\dot{\mathbf{U}}^{\jmath}$ are satisfied in $K_{0}\left(\dot{\mathfrak{U}}^{3}\right)$. For (2.6) $-(2.8)$ this follows from the same argument as in [KL10,

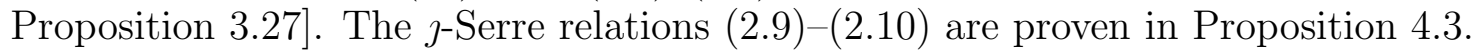

Our goal in this subsection is to show that the functor $\aleph:{ }_{\mathcal{A}} \dot{\mathbf{U}}^{\jmath} \rightarrow K_{0}(\dot{\mathfrak{U}})$ ) is full (see Proposition 4.8). We achieve this by showing that the graded category associated with a filtration on $\mathfrak{U}^{\mathfrak{j}}$ is equivalent to the KLR categorification of the positive half of ${ }_{\mathcal{A}} \dot{\mathbf{U}}$. Let us explain this in details.

Fix $\lambda \in \mathrm{X}_{\jmath}$. Recall from (2.1) and (2.4) that $\mathbb{I}=\mathbb{I}^{\jmath} \cup-\mathbb{I}^{\jmath}$, and that we write $\mathcal{E}_{-i}:=\mathcal{F}_{i}$ for $i \in \mathbb{I}^{\jmath}$. Given a sequence $\mathbf{i}=\left(i_{1}, i_{2}, \ldots, i_{m}\right) \in \mathbb{I}^{m}$ we write $\mathcal{E}_{\mathbf{i}}=\mathcal{E}_{i_{1}} \mathcal{E}_{i_{2}} \cdots \mathcal{E}_{i_{m}}$. Consider the category

$$
\mathcal{C}=\bigoplus_{\mu \in \mathrm{X}_{\jmath}} \dot{\mathfrak{U}} \mathfrak{\jmath}(\lambda, \mu)
$$


We will always view $\mathcal{E}_{\mathbf{i}}$ as the object $\mathcal{E}_{\mathbf{i}} 1_{\lambda}$ in $\mathcal{C}$, hence omit $1_{\lambda}$ from the notation. Since $\aleph$ is the identity on objects, it is enough to prove that $K_{0}(\mathcal{C})$ is in the image of $\aleph$. The category $\mathcal{C}$ is Krull-Schmidt, hence its Grothendieck group is a free $\mathcal{A}$-module generated by indecomposable objects. Up to a grading shift, any indecomposable object $P \in \mathcal{C}$ is a direct summand of $\mathcal{E}_{\mathbf{i}}$ for some sequence $\mathbf{i}$. We define the width of $P$ to be the minimum length of such a sequence $\mathbf{i}$. We define the width of a morphism $\beta: u \rightarrow v$ in $\mathcal{C}$ to be the minimal $m$ such that $\beta$ factors through a sum of objects of width $\leq m$. Let $\mathcal{C}_{\leqslant m}$ (respectively, $\mathcal{C}_{<m}$ ) be the full subcategory of $\mathcal{C}$ generated by indecomposable objects of width $\leqslant m$ (respectively of width $<m$ ). The quotient category $\operatorname{gr}^{m} \mathcal{C}=\mathcal{C}_{\leqslant m} / \mathcal{C}_{<m}$ is the additive category with the same objects as in $\mathcal{C}_{\leqslant m}$, and Hom-space given by the quotient of the Hom-space in $\mathcal{C}_{\leqslant m}$ by the 2 -sided ideal of morphisms of width $<m$. The indecomposable objects in $\operatorname{gr}^{m} \mathcal{C}$ are in bijection with those in $\mathcal{C}$ of length $m$. Hence we have $K_{0}(\mathcal{C})=K_{0}(\operatorname{gr} \mathcal{C})$, where $\operatorname{gr} \mathcal{C}=\bigoplus_{m \geqslant 0} \operatorname{gr}^{m} \mathcal{C}$

Let $\mathcal{C}^{+}$be the monoidal category (under induction) of graded projective modules over the quiver Hecke algebra of type $A_{2 r}$ (with $\mathbb{I}$ identified with the Dynkin diagram of this type). This is a graded $\mathbf{k}$-linear monoidal category with objects generated by $E_{i}, i \in \mathbb{I}$, and the morphisms generated by

$$
x=\uparrow_{i}^{\uparrow}: E_{i} \rightarrow E_{i}\{-2\}, \quad \tau=\searrow_{i}: E_{i} E_{j} \rightarrow E_{j} E_{i}\left\{\left\langle\alpha_{i}^{\vee}, \alpha_{j}\right\rangle\right\}
$$

subject to quiver Hecke relations (3.4)-(3.6). By [KL09, Proposition 3.18], $K_{0}\left(\mathcal{C}^{+}\right)$is isomorphic to ${ }_{\mathcal{A}} \mathbf{U}^{+}$, the positive part of ${ }_{\mathcal{A}} \dot{\mathbf{U}}$ generated by $E_{i}^{(a)}, i \in \mathbb{I}$. Let $\Pi_{\lambda}$ be the polynomial ring in the commuting variables (for $i \in \mathbb{I}^{\jmath}$ )

$$
s \bigcup_{i}^{\lambda} \lambda: 1_{\lambda} \rightarrow 1_{\lambda}\left\{-2\left(s+1-\left\langle{ }^{\theta} \alpha_{i}^{\vee}, \lambda\right\rangle\right)\right\} \text {, for } s \geq\left\langle{ }^{\theta} \alpha_{i}^{\vee}, \lambda\right\rangle-1
$$

Then $K_{0}\left(\Pi_{\lambda}\right)=\mathcal{A}$ and we have $K_{0}\left(\mathcal{C}^{+} \otimes \Pi_{\lambda}\right) \simeq K_{0}\left(\mathcal{C}^{+}\right)$; see [KL10, Proposition 3.35] for more details.

Lemma 4.7. There is a full functor $\zeta: \mathcal{C}^{+} \otimes \Pi_{\lambda} \rightarrow \operatorname{gr} \mathcal{C}$ which sends $E_{\mathbf{i}}$ to $\mathcal{E}_{\mathbf{i}}$, for all sequences $\mathbf{i}$ and a diagram $D$ to $(-1)^{d}$ times that diagram with the orientation on the $i$-th strands reversed for all $i<0$. Here $d$ is the number of dots on strands whose orientation was reversed.

Proof. Let us first show that $\zeta$ is well defined. It is enough to show that the assignment in the lemma defines an algebra homomorphism from $R_{m}=\operatorname{End}_{\mathcal{C}^{+} \otimes \Pi_{\lambda}}\left(\bigoplus_{|\mathbf{i}|=m} E_{\mathbf{i}} 1_{\lambda}\right)$ to $S_{m}=$ $\operatorname{End}_{\mathrm{gr}^{m} \mathcal{C}}\left(\bigoplus_{|\mathbf{i}|=m} \mathcal{E}_{\mathbf{i}} 1_{\lambda}\right)$. In other words $\zeta(x)$ and $\zeta(\tau)$ satisfy the quiver Hecke relations for all $i, j \in \mathbb{I}$. This is obvious if $i$ and $j$ have the same sign. Next, note that indecomposable summands of $\mathcal{E}_{m}=\bigoplus_{|\mathbf{i}|=m} \mathcal{E}_{\mathbf{i}}$ are in bijection with primitive idempotents in $A=\operatorname{End}_{\mathcal{C}}\left(\mathcal{E}_{m}\right)$. By definition $S_{m}$ is the quotient of $A$ by the 2-sided ideal $I$ of morphisms of width $<m$. Now, relations (3.14), (3.15), (3.17) in $\mathfrak{U}^{\mathfrak{J}}$ modulo morphisms of width $<2$ give the quiver Hecke relations for $i, j$ of different sign. We deduce that $\zeta: R_{m} \rightarrow A / I=S_{m}$ is well defined.

It remains to show that $\zeta: R_{m} \rightarrow S_{m}$ is surjective. To this end, note that $\operatorname{End}_{\mathcal{C}}\left(\bigoplus_{|\mathbf{i}|=m} \mathcal{E}_{\mathbf{i}} 1_{\lambda}\right)$ is spanned by diagrams where no pair of strands cross twice and all bubbles are at far left. Every such diagram either has no cups or caps (except in bubbles) or has width $<m$. Thus $S_{m}$ is spanned over $\Pi_{\lambda}$ by diagrams without cups or caps. The image of $\zeta$ contains all diagrams of this type, so the surjectivity follows. 
Proposition 4.8. The functor $\aleph:{ }_{\mathcal{A}} \dot{\mathbf{U}}^{\jmath} \rightarrow K_{0}(\dot{\mathfrak{U} j})$ is full, i.e., for any $\lambda, \mu \in \mathrm{X}_{\jmath}$, it defines a surjective map

$$
{ }_{\mathcal{A}} \dot{\mathbf{U}}^{\jmath}(\lambda, \mu) \rightarrow K_{0}(\dot{\mathfrak{U}} \jmath(\lambda, \mu)) .
$$

It will be proved in Theorem 6.5 that $\aleph:{ }_{\mathcal{A}} \dot{\mathbf{U}}^{\jmath} \rightarrow K_{0}\left(\dot{\mathfrak{U}}^{\jmath}\right)$ is in fact an equivalence.

Proof. The same argument as in [KL10, Section 3.8.3] implies that the map

$$
K_{0}(\zeta):{ }_{\mathcal{A}} \mathbf{U}^{+} \simeq K_{0}\left(\mathcal{C}^{+} \otimes \Pi_{\lambda}\right) \longrightarrow K_{0}(\operatorname{gr} \mathcal{C})
$$

is surjective.

By [Lu90, 7.8], the algebra ${ }_{\mathcal{A}} \mathbf{U}^{+}$has an $\mathcal{A}$-basis $\mathbf{M}$ whose elements are monomials in divided powers $E_{i}^{(a)}$. Hence $K_{0}(\zeta)$ maps them to a spanning set of the $\mathcal{A}$-module $K_{0}($ gr $\mathcal{C})$ which are monomials in $\mathcal{E}_{i}^{(a)}$ for $i \in \mathbb{I}$. By the isomorphism $K_{0}(\mathcal{C})=K_{0}(\operatorname{gr} \mathcal{C})$, we deduce that $K_{0}(\mathcal{C})$ is also spanned by these monomials, which are contained in the image of $\aleph$. Hence $\aleph$ is full.

Let $\mathbf{M}$ be a monomial basis of ${ }_{\mathcal{A}} \mathbf{U}^{+}$in the sense of [Lu90, 7.8]. Let

$$
{ }_{\mathcal{A}} \dot{\mathbf{U}}^{\jmath}(\lambda,-)=\bigoplus_{\mu \in \mathrm{X}_{\mathcal{J}}} \dot{\mathbf{U}}^{\jmath}(\lambda, \mu)
$$

For $m=E_{i_{1}}^{\left(c_{1}\right)} E_{i_{2}}^{\left(c_{2}\right)} \ldots$, we write $m^{\jmath}=\mathcal{E}_{i_{1}}^{\left(c_{1}\right)} \mathcal{E}_{i_{2}}^{\left(c_{2}\right)} \ldots$ accordingly. The corollary below was known only for some special choice of the monomial bases (see the proof of [BKLW], Theorem 4.7]).

Corollary 4.9. For any $\lambda \in \mathrm{X}_{\jmath}$, the assignment $m \mapsto m^{\jmath}$ for all $m \in \mathbf{M}$ yields an isomorphism of $\mathcal{A}$-modules

$$
\varsigma: \dot{\mathbf{U}}^{+} \stackrel{\sim}{\rightarrow}{ }_{\mathcal{A}} \dot{\mathbf{U}}^{\jmath}(\lambda,-) .
$$

Proof. The proof of [Ko14, Proposition 6.2] adapted to the idempotented algebra case implies that $\varsigma$ is an isomorphism after base change to $\mathbb{Q}(q)$. Since ${ }_{\mathcal{A}} \dot{\mathbf{U}}^{+}$is free over $\mathcal{A}$, we get injectivity of $\varsigma$. The surjectivity follows from the proof of Proposition 4.8 .

\subsection{Control from the Grothendieck group.}

Proposition 4.10. Let $\mathfrak{C}$ be any idempotent complete 2-category. A 2-functor $\phi: \widetilde{\mathfrak{U}}^{j} \rightarrow \mathfrak{C}^{\mathfrak{c}}$ induces a 2-functor $\dot{\mathfrak{U}} \rightarrow \mathfrak{C}$ if and only if the classes of $\phi\left(\mathcal{E}_{\diamond} 1_{\lambda}\right)$ and $\phi\left(\mathcal{F}_{\diamond} 1_{\lambda}\right)$ satisfy the $\jmath$-Serre relations (2.9)-(2.10) in $K_{0}(\mathfrak{C})$, for any $\lambda \in \mathrm{X}_{\jmath}$.

Proof. Let $\Xi$ denote the right hand side of (3.17). Since $\mathfrak{C}$ is idempotent complete, the 2-functor $\phi$ factors through $\dot{\mathfrak{U}}$ if and only if $\phi(\Xi)$ is identity for all $\lambda$. We may assume $\lambda_{\diamond} \geqslant 2$, as the proof for the other cases are similar. By Proposition 4.3, we have a split surjective map

$$
\Gamma: \mathcal{F}_{\diamond} \mathcal{E}_{\diamond} \mathcal{F}_{\diamond} 1_{\lambda} \longrightarrow \mathcal{F}_{\diamond}^{(2)} \mathcal{E}_{\diamond} \oplus\left(\mathcal{E}_{\diamond} \mathcal{F}_{\diamond}^{(2)}-\left(\lambda_{\diamond}-2\right) \mathcal{F}_{\diamond}\right) 1_{\lambda} \oplus \mathcal{F}_{\diamond} 1_{\lambda}^{\oplus\left[\lambda_{\diamond}\right]}
$$

in the idempotent completion of $\widetilde{\mathfrak{U}}^{\jmath}$ given by $\Gamma=B_{1} \oplus\left(\kappa \cdot B_{2}\right) \oplus P_{0}^{\prime} \oplus \ldots \oplus P_{\lambda_{\diamond}-1}$. A splitting is provided by $\Gamma^{\prime}=C_{1}+\left(C_{2} \cdot \kappa\right)+I_{0}+\ldots+I_{\lambda_{\diamond}-1}$, that is, $\Gamma \circ \Gamma^{\prime}=$ id; see the proof of Proposition 4.3 for the notation. Furthermore, we have $\Xi=\Gamma^{\prime} \circ \Gamma$ by Proposition A.22, It follows that $\Xi$ is an idempotent in $\widetilde{\mathfrak{U}}^{\jmath}$. Hence $\phi(\Xi)=1$ if and only if $\phi(\Gamma)$ is an isomorphism, if and only if the $\jmath$-Serre relation holds in $K_{0}(\mathfrak{C})$. 


\section{The Schur 2-CATEgory}

5.1. Soergel bimodules. We start by reviewing some standard facts about singular Soergel bimodules from Wil11 that will be used below.

Let $\mathrm{W}$ be a Weyl group with the set of simple reflections $\mathrm{S}$, and let $\mathfrak{t}$ be a faithful $\mathbf{k}$-linear reflection representation of $\mathrm{W}$. Consider the graded ring of symmetric product $R=S(\mathfrak{t})$ with elements in $\mathfrak{t}$ in degree 2. Then the $\mathrm{W}$-action on $\mathfrak{t}$ induces a homogeneous action on $R$.

Given $I \subset \mathrm{S}$, let $\mathrm{W}_{I}$ be the parabolic subgroup generated by $I$. We write $R^{I}=R^{\mathrm{W}_{I}}$ for the invariants in $R$ under $\mathrm{W}_{I}$. Given a triple $I \supset K \subset J$, we view $R^{K}$ as an $\left(R^{I}, R^{J}\right)$-bimodule via the canonical embeddings $R^{I} \hookrightarrow R^{K} \hookleftarrow R^{J}$.

Let $\mathfrak{B} i m_{\mathrm{W}}$ denote the 2-category with objects consisting of subsets in $\mathrm{S}$, and $\mathfrak{B i m}_{\mathrm{W}}(J, I)=$ $R^{I}$-gmod- $R^{J}$, with composition given by tensor product of bimodules. The category of singular Soergel bimodules $\mathbf{S} \mathfrak{B} i m_{\mathrm{W}}$ is the full 2-subcategory of $\mathfrak{B} i m_{\mathrm{W}}$ generated by the same objects and 1-morphisms $R^{I} \hookrightarrow R^{K} \hookleftarrow R^{J}$ for all triples $I \supset K \subset J$. In particular, indecomposable objects in $\mathbf{S} \mathfrak{B} i_{\mathrm{W}}(J, I)$ are direct summands of

$$
R^{I_{1}} \otimes_{R^{J_{1}}} R^{I_{2}} \otimes_{R^{J_{2}}} \ldots \otimes_{R^{J_{n-1}}} R^{I_{n}}
$$

for certain sequence $I=I_{1} \subset J_{1} \supset I_{2} \subset J_{2} \supset \ldots \subset J_{n-1} \supset I_{n}=J$. Let $\psi: \mathbf{S B i m}$ W $\rightarrow \mathbf{S B i m}$ W be the duality given by taking graded duals. In particular $\psi \circ\{1\}=\{-1\} \circ \psi$.

Consider the Schur algebroid $\mathbf{S}_{\mathrm{W}}$ associated with $\mathrm{W}$. This is an $\mathcal{A}$-linear category with objects finitary $I \subset S$, and morphisms from $I$ to $J$ given by intersections of parabolic modules over the Hecke algebra $\mathbf{H}_{\mathrm{W}}$, see [Wil11, Definition 2.7]. Note that $\mathbf{S}_{\mathrm{W}}(\emptyset, \emptyset)=\mathbf{H}_{\mathrm{W}}$.

Theorem 5.1. Soe07, Wil11

(a) There is an equivalence of categories

$$
\kappa: K_{0}\left(\mathbf{S B i m}{ }_{\mathrm{W}}\right) \stackrel{\sim}{\rightarrow} \mathbf{S}_{\mathrm{W}}
$$

which is the identity on objects, and is given by the character map on morphisms.

(b) The equivalence $\kappa$ intertwines $\psi$ with the bar involution on $\mathbf{S}_{\mathrm{W}}$.

(c) For each element $w$ in $\mathrm{W}_{I} \backslash \mathrm{W} / \mathrm{W}_{J}$, there is a unique self-dual indecomposable object $\mathcal{B}_{w}$ in $\mathbf{S} \mathfrak{B i m}{ }_{\mathrm{W}}(I, J)$ characterized by a support condition. These objects form a complete and irredundant set of indecomposable objects in $\mathbf{S} \mathfrak{B} i_{\mathrm{W}}(I, J)$ up to isomorphism and grading shift.

(d) If the residue field of $\mathbf{k}$ has characteristic zero, then $\kappa$ sends $\left[\mathcal{B}_{w}\right]$ to the element in the canonical basis of $\mathbf{S}_{\mathrm{W}}(I, J)$ indexed by $w$.

We have a geometric interpretation of $\mathbf{S} \mathfrak{B} i m_{W}$ as follows. Let $\mathrm{G}$ be a connected reductive group with Weyl group W. Fix a Borel subgroup B and a maximal torus T. Take $\mathfrak{t}$ to be the k-module spanned by the characters of T. For $I \subset \mathrm{S}$ let $\mathrm{B} \subset \mathrm{P}_{I} \subset \mathrm{G}$ be the parabolic subgroup corresponding to $I$. The diagonal G-orbits in $\mathrm{G} / \mathrm{P}_{I} \times \mathrm{G} / \mathrm{P}_{J}$ are parametrized by $\mathrm{W}_{I} \backslash \mathrm{W} / \mathrm{W}_{J}$. Then $\mathbf{S}_{\mathrm{W}}(I, J)$ is the generic algebra of $\mathcal{A}$-valued functions on $\mathrm{G} \backslash\left(\mathrm{G} / \mathrm{P}_{I} \times \mathrm{G} / \mathrm{P}_{J}\right)$ arising from the convolution product of functions on $\mathrm{P}_{I}\left(\mathbb{F}_{\mathbf{q}^{2}}\right) \backslash \mathrm{G}\left(\mathbb{F}_{\mathbf{q}^{2}}\right) / \mathrm{P}_{J}\left(\mathbb{F}_{\mathbf{q}^{2}}\right)$ over finite fields $\mathbb{F}_{\mathbf{q}^{2}}$.

Proposition 5.2. The category $\mathbf{S} \mathfrak{B} i m_{W}(I, J)$ is equivalent to the category of $\mathrm{G}$-equivariant parity complexes on $\mathrm{G} / \mathrm{P}_{I} \times \mathrm{G} / \mathrm{P}_{J}$, with $\mathcal{B}_{w}$ corresponding to the unique parity sheaf $\mathcal{E}_{w}$ whose support is the closure of the orbit $O_{w}$. 
All the machinery needed to prove this result is given in [JMW14, 4.1] but it is not stated there. In type A, this is discussed in greater detail in [W15b, Theorem 6], and the proof is identical in other types. When the residue field of $\mathbf{k}$ has characteristic zero, the parity sheaf $\mathcal{E}_{w}$ is an intersection cohomology complex.

5.2. Schur category in type B/C. From now on let $\mathrm{W}=\mathrm{W}_{m}$ be the Weyl group of type $B_{m} / C_{m}$ with simple reflections $\mathrm{S}=\left\{s_{0}, s_{1}, \ldots, s_{m-1}\right\}$. Take $\mathfrak{t}=\bigoplus_{i=1}^{m} \mathbf{k} t_{i}$ with $s_{i}$ acting by permuting $t_{i}$ and $t_{i+1}$, and $s_{0}\left(t_{i}\right)=(-1)^{\delta_{i, 1}} t_{i}$. We have

$$
R=S(\mathfrak{t})=\mathbf{k}\left[t_{1}, \ldots, t_{m}\right]
$$

Let $\mathrm{G}=\mathrm{SO}(\mathrm{V})$ with $\mathrm{V}=\mathbb{C}^{2 m+1}$ equipped with a standard nondegenerate symmetric bilinear form.

Recall $\mathbb{I}_{r}^{\jmath}$ from (2.4). Let $\Sigma_{r, m}$ be the set of (weakly) increasing maps from $\mathbb{I}_{r}^{\jmath} \rightarrow[0, m]$, and we write an element $\mathbf{a} \in \Sigma_{r, m}$ as an increasing sequence $\mathbf{a}=\left(a_{\diamond}, a_{\diamond+1}, \ldots, a_{\diamond+r-1}\right)$. To $\mathbf{a} \in \Sigma_{r, m}$ we associate the subset $I_{\mathbf{a}}$ of $\mathrm{S}$ with $s_{a_{p}}$ removed for all $p$ such that $a_{p}<m$. Note that if $r \geqslant m$, then every subset of $\mathrm{S}$ is of this form. Given $\mathbf{a} \in \Sigma_{r, m}$ and $i \in \mathbb{I}_{r}^{\jmath}$ let

$$
\begin{array}{rlrl}
\mathbf{a}^{+i} & =\left(\ldots, a_{i-1}, a_{i}, a_{i}+1, a_{i+1}, \ldots\right), & \mathbf{a}^{-i} & =\left(\ldots, a_{i-1}, a_{i}-1, a_{i}, a_{i+1}, \ldots\right), \\
& & & \\
+{ }_{i} \mathbf{a} & =\left(\ldots, a_{i-1}, a_{i}+1, a_{i+1}, \ldots\right), & { }_{-i} \mathbf{a}=\left(\ldots, a_{i-1}, a_{i}-1, a_{i+1}, \ldots\right) .
\end{array}
$$

For any sequence a of $r$ integers, we write

$$
R^{\mathbf{a}}= \begin{cases}R^{I_{\mathbf{a}}}, & \text { if } \mathbf{a} \in \Sigma_{r, m}, \\ 0, & \text { if } \mathbf{a} \notin \Sigma_{r, m} .\end{cases}
$$

Then $R^{\mathbf{a}^{ \pm i}}$ is naturally an $\left(R^{ \pm i} \mathbf{a}, R^{\mathbf{a}}\right)$-bimodule. Indeed $\mathbf{a}^{ \pm i}$ is a sequence of $r+1$ integers. If it belongs to $\Sigma_{r+1, m}$, then ${ }_{ \pm i} \mathbf{a}$ also belongs to $\Sigma_{r, m}$, moreover $I_{ \pm i} \mathbf{a} \supset I_{\mathbf{a}^{ \pm i}} \subset I_{\mathbf{a}}$, hence $R^{\mathbf{a}^{ \pm i}}$ is an $\left(R^{ \pm i} \mathbf{a}, R^{\mathbf{a}}\right)$-bimodule. If $\mathbf{a}^{ \pm i} \notin \Sigma_{r+1, m}$, then $R^{\mathbf{a}^{ \pm i}}=0$, the statement is trivial. The partial flag variety $\imath \mathrm{Gr}_{\mathbf{a}}:=\mathrm{G} / \mathrm{P}_{I_{\mathrm{a}}}$ is the variety of isotropic flags

$$
0=F_{-r-\diamond} \subset \ldots \subset F_{-\diamond} \subset F_{\diamond} \subset \ldots \subset F_{\diamond+r}=\mathrm{V}
$$

such that $F_{p}=F_{-p}^{\perp}, \operatorname{dim} F_{-p}=m-a_{p}$ (and so $\operatorname{dim} F_{p}=m+1+a_{p}$ ) for all $p \in \mathbb{I}^{\jmath}$.

Consider the following 1-morphisms in $\mathbf{S} \mathfrak{B} i m_{\mathrm{W}}$ for all $i \in \mathbb{I}_{r}^{\jmath}$

$$
\begin{array}{r}
1_{\mathbf{a}}:=R^{\mathbf{a}} \in R^{\mathbf{a}} \text {-gmod- } R^{\mathbf{a}}, \\
\mathscr{E}_{i} 1_{\mathbf{a}}:=R^{\mathbf{a}^{+i}\left\{1+a_{i}-a_{i+1}\right\}} \in R^{+i} \mathbf{a} \text {-gmod- } R^{\mathbf{a}}, \\
\mathscr{F}_{i} 1_{\mathbf{a}}:=R^{\mathbf{a}^{-i}\left\{1+a_{i-1}-a_{i}\right\}} \in R^{-i \mathbf{a}} \text {-gmod- } R^{\mathbf{a}} .
\end{array}
$$

Here and below, we use the convention

$$
a_{-\diamond}=-a_{\diamond}, \quad a_{\diamond+r}=m .
$$

We define the Schur 2-category $\mathfrak{F}_{r, m}$ as the locally fully faithful monoidal 2-subcategory of S $\mathfrak{B} i m_{\mathrm{W}}$ with object set $\Sigma_{r, m}$ and with 1-morphisms given by direct summands of direct sums of products of $1_{\mathbf{a}}, \mathscr{E}_{i} 1_{\mathbf{a}}, \mathscr{F}_{i} 1_{\mathbf{a}}$, for $\mathbf{a} \in \Sigma_{r, m}, i \in \mathbb{I}_{r}^{\jmath}$. Recall that the locally fully faithfulness here means that we impose for any 1-morphisms $M, N$ in $\mathfrak{F}_{r, m}$ that $\operatorname{Hom}_{\mathfrak{F}_{r, m}}(M, N)=$ $\operatorname{Hom}_{\mathbf{S} \mathfrak{B} i m_{\mathrm{W}}}(M, N)$. 
Recall that the Schur category $\mathbf{S}_{r, m}^{\jmath}$ (also called the SSchur algebra) is the category with object set $\Sigma_{r, m}$ and $\mathbf{S}_{r, m}^{\jmath}(\mathbf{a}, \mathbf{b}):=\mathbf{S}_{W}\left(I_{\mathbf{a}}, I_{\mathbf{b}}\right)$, for $\mathbf{a}, \mathbf{b} \in \Sigma_{r, m}$. Similarly, we have a fully faithful functor $\mathbf{S}_{r, m}^{\jmath} \rightarrow \mathbf{S}_{W}$. For $\mathbf{a} \in \Sigma_{r, m}$, let $\lambda(\mathbf{a}) \in \mathbf{X}_{\text {J }}$ be such that

$$
\left\langle{ }^{\theta} \alpha_{i}, \lambda(\mathbf{a})\right\rangle=-a_{i-1}+2 a_{i}-a_{i+1}, \quad \forall i \in \mathbb{I}_{r}^{\jmath} .
$$

In this way $\Sigma_{r, m}$ can be viewed as a subset of $\mathrm{X}_{\jmath}$. It is easy to see that $\lambda(\mathbf{a})=a_{\diamond} \varepsilon_{0}+\sum_{i=\diamond+1}^{r+\diamond}\left(a_{i}-\right.$ $\left.a_{i-1}\right) \varepsilon_{i-\diamond} \in X_{\jmath}$.

By [BKLW, Proposition 3.1, Corollary 3.13], there is a well-defined functor

$$
\gamma: \dot{\mathbf{U}}^{\jmath} \longrightarrow \mathbf{S}_{r, m}^{\jmath}
$$

such that for $\lambda \in \mathrm{X}_{\text {j }}$ we have $\gamma(\lambda)=\mathbf{a}$ if there exists $\mathbf{a} \in \Sigma_{r, m}$ (which must be unique by (5.1)) such that $\lambda=\lambda(\mathbf{a})$, and $\gamma(\lambda)=0$ otherwise. The images $\mathbf{e}_{i}=\gamma\left(\mathcal{E}_{i}\right), \mathbf{f}_{i}=\gamma\left(\mathcal{F}_{i}\right)$, for $i \in \mathbb{I}_{r}^{J}$, generate $\mathbf{S}_{r, m}^{\jmath} \otimes_{\mathcal{A}} \mathbb{Q}(q)$.

\section{Proposition 5.3.}

(a) For $\mathbf{a}, \mathbf{b} \in \Sigma_{r, m}$, we have $\mathfrak{F}_{r, m}(\mathbf{a}, \mathbf{b})=\mathbf{S} \mathfrak{B i m} \mathrm{W}(\mathbf{a}, \mathbf{b})$.

(b) There is an equivalence $\kappa: K_{0}\left(\mathfrak{F}_{r, m}\right) \stackrel{\sim}{\rightarrow} \mathbf{S}_{r, m}^{\jmath}$ such that

$$
\kappa(\mathbf{a})=\mathbf{a}, \quad \kappa\left(\left[\mathscr{E}_{i} 1_{\mathbf{a}}\right]\right)=\mathbf{e}_{i} 1_{\mathbf{a}}, \quad \kappa\left(\left[\mathscr{F}_{i} 1_{\mathbf{a}}\right]\right)=\mathbf{f}_{i} 1_{\mathbf{a}} .
$$

It intertwines the duality $\psi$ on $\mathfrak{F}_{r, m}$ and the bar involution.

(c) If the residue field of $\mathbf{k}$ has characteristic zero, then $\kappa$ sends self-dual indecomposable objects in $\mathfrak{F}_{r, m}$ to canonical basis in $\mathbf{S}_{r, m}^{J}$.

Proof. The nontrivial statement here is part (a). The rest follows automatically from Theorem 5.1 and (a). To prove (a), let $\mathfrak{F}_{r, m}^{\prime}$ be the 2-subcategory of $\mathbf{S} \mathfrak{B} i m_{\mathrm{W}}$ with object set $\Sigma_{r, m}$ and such that $\mathfrak{F}_{r, m}^{\prime}(\mathbf{a}, \mathbf{b})=\mathbf{S} \mathfrak{B} i m_{\mathrm{W}}(\mathbf{a}, \mathbf{b})$ for any $\mathbf{a}, \mathbf{b} \in \Sigma_{r, m}$. By definition, we have a locally fully faithful embedding $\iota: \mathfrak{F}_{r, m} \rightarrow \mathfrak{F}_{r, m}^{\prime}$, which sends indecomposable 1-morphisms to indecomposable ones. We must show that $\iota$ is full, that is all indecomposable 1-morphisms in $\mathfrak{F}_{r, m}^{\prime}$ are in the image. This is true if and only if the fully faithful functor $[\iota]: K_{0}\left(\mathfrak{F}_{r, m}\right) \hookrightarrow K_{0}\left(\mathfrak{F}_{r, m}^{\prime}\right)$ induced by $\iota$ is an equivalence. Note that the morphism spaces of both Grothendieck groups are free $\mathcal{A}$-modules with bases given by classes of indecomposable 1 -morphisms, hence $[\iota]$ is a split injection. Hence it is an equivalence if and only if it is so after base exchange from $\mathcal{A}$ to $\mathbb{Q}(q)$. Now, by Theorem [5.1, we have $K_{0}\left(\mathfrak{F}_{r, m}^{\prime}\right) \cong \mathbf{S}_{r, m}^{\jmath}$. Therefore $K_{0}\left(\mathfrak{F}_{r, m}^{\prime}\right) \otimes_{\mathcal{A}} \mathbb{Q}(q)$ is generated by $1_{\mathbf{a}}, \mathbf{e}_{i} 1_{\mathbf{a}}, \mathbf{f}_{i} 1_{\mathbf{a}}, i \in \mathbb{I}_{r}^{\jmath}$. By the definition of $\mathfrak{F}_{r, m}$, all these generators lie in the image of $[\iota]$. Therefore $[\iota]$ is an equivalence after base change to $\mathbb{Q}(q)$. Part (a) is proved.

Remark 5.4. We write $\mathfrak{F}_{r, m}^{\mathfrak{a}}$ for the 2-category defined with the same $R=S(\mathfrak{t})$ as for $\mathfrak{F}_{r, m}$ but with $\mathrm{W}_{m}$ replaced by the symmetric group $\mathfrak{S}_{m}=\left\langle s_{1}, s_{2}, \ldots, s_{m-1}\right\rangle$.

Remark 5.5. We will also consider a quotient $\mathbf{S} \dot{\mathfrak{U}}$ j of the 2-category $\dot{\mathfrak{U}}^{j}$ by setting to 0 all objects of weight which are not of the form $\lambda(\mathbf{a})$. The functor $\Gamma: \dot{\mathfrak{U}}^{j} \rightarrow \mathfrak{F}_{r, m}$ factors through this quotient. We claim that

$$
K_{0}\left(\mathbf{S} \dot{\mathfrak{U}}^{\jmath}\right) \cong \mathbf{S}_{r, m}^{\jmath}
$$

Indeed, consider the following composition of functors

$$
{ }_{\mathcal{A}} \dot{\mathbf{U}}^{\jmath} \rightarrow K_{0}\left(\dot{\mathfrak{U}}^{\jmath}\right) \rightarrow K_{0}\left(\mathbf{S} \dot{U}^{\jmath}\right) \rightarrow K_{0}\left(\mathfrak{F}_{r, m}\right) \stackrel{\sim}{\rightarrow} \mathbf{S}_{r, m}^{\jmath} .
$$


Since the map ${ }_{\mathcal{A}} \dot{\mathbf{U}}^{\jmath} \rightarrow \mathbf{S}_{r, m}^{\jmath}$ is surjective, with kernel generated by the objects not of the form $1_{\lambda(\mathbf{a})}$, see [BKLW, Prop 4.11, Lemma A.20, Thm A.21], we must have $K_{0}(\mathbf{S} \dot{\mathfrak{U} \jmath}) \cong \mathbf{S}_{r, m}^{\jmath}$.

5.3. Frobenius forms and Demazure operators. Let $I$ be a subset of $[1, m]$. Let $\Lambda_{I}$ be the ring of symmetric functions in $\left\{t_{i} ; i \in I\right\}$. For $p \geqslant 0$, let $e_{p, I}=e_{p}\left(t_{i} ; i \in I\right)$ be the $p$-th elementary symmetric polynomial, and let $h_{p, I}=h_{p}\left(t_{i} ; i \in I\right)$ be the $p$-th complete symmetric polynomial. They are defined by the following generating functions

$$
\sum_{p \geqslant 0} e_{p, I} z^{p}=\prod_{i \in I}\left(1+t_{i} z\right), \quad \sum_{p \geqslant 0} h_{p, I} z^{p}=\prod_{i \in I}\left(1-t_{i} z\right)^{-1}
$$

where $z$ is a formal variable. Note that

$$
\sum_{p+s=k}(-1)^{p} e_{p, I} h_{s, I}=\delta_{k, 0} .
$$

We also write $\Lambda_{I}^{\jmath}$ for the ring of symmetric functions in $\left\{t_{i}^{2} ; i \in I\right\}$, and $e_{p, I}^{\jmath}=e_{p}\left(t_{i}^{2} ; i \in I\right)$, $h_{p, I}^{\jmath}=h_{p}\left(t_{i}^{2} ; i \in I\right)$. For future use, we introduce the convention that $h_{p, I}^{\jmath}=0$ if $p \notin \mathbb{N}$.

Let $B$ be a k-algebra, and $A$ a finitely generated $B$-algebra which is free as a $B$-module. A $(B, B)$-linear map $\phi: A \rightarrow B$ is a Frobenius form if $A \rightarrow \operatorname{Hom}_{B}(A, B), a \mapsto(b \mapsto \phi(a b))$ is an isomorphism. The Casimir element associated with $\phi$ is an element $\pi \in\left(A \otimes_{B} A\right)^{A}$ such that $(\phi \otimes 1)(\pi)=(1 \otimes \phi)(\pi)=1$. The restriction from $A$ to $B$ is left adjoint to $A \otimes_{B}-$ with counit given by $\phi$ and unit given by $A \mapsto A \otimes_{B} A, 1 \mapsto \pi$. See for example [R08, Section 2.3] for more details.

We recall some basic facts on Demazure operators. Let $\alpha_{s_{0}}^{\vee}=-2 t_{1}$, and $\alpha_{s_{p}}^{\vee}=t_{p}-t_{p+1}$ for $p>0$. For any reflection $\tau \in \mathrm{W}$, let $\alpha_{\tau}^{\vee}$ denote the corresponding positive coroot. For $s \in \mathrm{S}$ the Demazure operator $\partial_{s}: R \rightarrow R$ is given by

$$
\partial_{s}(f)=\frac{f-s(f)}{\alpha_{s}^{\vee}}, \quad \forall f \in R
$$

For $w \in \mathrm{W}$ let $\partial_{w}=\partial_{s_{i_{1}}} \partial_{s_{i_{2}}} \ldots$ for a reduced expression $s_{i_{1}} s_{i_{2}} \ldots$ of $w$. It is a well defined operator of degree $-2 \ell(w)$, where $\ell(w)$ is the length of $w$. For $I \subset \mathrm{S}$, let $w_{I}$ be the longest element in $W_{I}$. Let $d_{I}$ be the product of $\alpha_{\tau}^{\vee}$ for all reflections $\tau$ in $\mathrm{W}_{I}$. Then

$$
\partial_{w_{I}}(f)=\frac{1}{\left|\mathrm{~W}_{I}\right|} \sum_{w \in \mathrm{W}_{I}} w\left(d_{I}^{-1} f\right), \quad \forall f \in R .
$$

It yields a Frobenius form $\partial_{w_{I}}: R\left\{-2 \ell\left(w_{I}\right)\right\} \rightarrow R^{I}$, see e.g. [Wil08, Section 3.1].

Remark 5.6. As mentioned before, everything we have done up to this point only depends on the Weyl group, not on the underlying root system. However, $d_{I}$ does depend on the choice of a root system; if we let $d_{I}^{\prime}$ be the corresponding product of coroots in type $\mathrm{C}$ rather than type $\mathrm{B}, d_{I}$ and $d_{I}^{\prime}$ differ by a power of 2 .

For $I^{\prime} \subset I \subset \mathrm{S}$, let $w_{I, I^{\prime}}=w_{I} w_{I^{\prime}}^{-1}, d_{I, I^{\prime}}=d_{I} / d_{I^{\prime}}$. Let $W^{I, I^{\prime}}$ be the set of minimal coset representatives of $W_{I} / W_{I^{\prime}}$. Then

$$
\partial_{w_{I, I^{\prime}}}: R^{I^{\prime}}\left\{-2 \ell\left(w_{I^{\prime}} w_{I}^{-1}\right)\right\} \longrightarrow R^{I}, \quad f \mapsto \frac{1}{\left|\mathrm{~W}_{I} / \mathrm{W}_{I^{\prime}}\right|} \sum_{w \in \mathrm{W}^{I, I^{\prime}}} w\left(d_{I, I^{\prime}}^{-1} f\right)
$$


is also a Frobenius form by [R08, Lemma 2.12]. Let us compute this form in some useful examples.

Example 5.7. Let $1 \leqslant a<b \leqslant m$.

(a) Consider $I^{\prime}=\left\{s_{p} ; p \in[a+1, b-1]\right\} \subset I=\left\{s_{p} ; p \in[a, b-1]\right\}$. Then $w_{I, I^{\prime}}=s_{b-1} \ldots s_{a}$. We will write $\partial_{[b, a]}=\partial_{w_{I, I^{\prime}}}$. The Frobenius form (5.5) in this case becomes

$$
\partial_{[b, a]}: \mathbf{k}\left[t_{a}\right] \otimes \Lambda_{[a+1, b]}\{2(a-b)\} \longrightarrow \Lambda_{[a, b]}, \quad f \mapsto \sum_{p=a}^{b} s_{(a, p)}\left(f \prod_{u=a+1}^{b}\left(t_{a}-t_{u}\right)^{-1}\right)
$$

Here we have ignored the variables $t_{i}$ for $i \notin[a, b]$, since $\partial_{[a, b]}$ acts trivially on them. An easy computation shows that $\partial_{[b, a]}\left(t_{a}^{k}\right)=h_{k-b+a,[a, b]}$ for all $k$. Therefore $\left\{t_{a}^{k}\right\}$ and $\left\{(-1)^{r} e_{r,[a+1, b]}\right\}$ are dual bases with respect to the Frobenius form $\partial_{[b, a]}$. Hence the Casimir element is given by

$$
\pi_{[b, a]}=\sum_{r=0}^{b-a} t_{a}^{b-a-r} \otimes(-1)^{r} e_{r,[a+1, b]} .
$$

(b) Consider $I^{\prime}=\left\{s_{p} ; p \in[a, b-2]\right\} \subset I=\left\{s_{p} ; p \in[a, b-1]\right\}$. Then $w_{I, I^{\prime}}=s_{a} \ldots s_{b-1}$. We will write $\partial_{[a, b]}=(-1)^{b-a} \partial_{w_{I, I^{\prime}}}$. The Frobenius form (5.5) in this case becomes

$$
\partial_{[a, b]}: \Lambda_{[a, b-1]} \otimes \mathbf{k}\left[t_{b}\right]\{2(a-b)\} \longrightarrow \Lambda_{[a, b]}, \quad f \mapsto \sum_{p=a}^{b} s_{(p, b)}\left(f \prod_{u=a}^{b-1}\left(t_{b}-t_{u}\right)^{-1}\right) .
$$

We have $\partial_{[a, b]}\left(t_{b}^{k}\right)=h_{k-b+a,[a, b]}$ for all $k$, and the Casimir element is

$$
\pi_{[a, b]}=\sum_{r=0}^{b-a}\left(t_{b}\right)^{b-a-r} \otimes(-1)^{r} e_{r,[a, b-1]} .
$$

(c) Consider $I^{\prime}=\left\{s_{p} ; p \in[0, a-2]\right\} \subset I=\left\{s_{p} ; p \in[0, a-1]\right\}$. We have $w_{I, I^{\prime}}=$ $s_{a-1} \ldots s_{1} s_{0} s_{1} \ldots s_{a-1}$. Call this element $\gamma_{a}$. Note that $\gamma_{a}\left(t_{i}\right)=(-1)^{\delta_{i, a}} t_{i}$. Write $\tilde{\partial}_{[1, a]}=$ $(-1)^{a} \partial_{w_{I, I^{\prime}}}$. The Frobenius form (5.5) in this case becomes

$$
\begin{aligned}
\tilde{\partial}_{[1, a]}: \Lambda_{[1, a-1]}^{\jmath} \otimes \mathbf{k}\left[t_{a}\right]\{2(1-2 a)\} & \longrightarrow \Lambda_{[1, a]}^{\jmath}, \\
f & \mapsto \sum_{p=1}^{a} s_{(p, a)}\left(1+\gamma_{a}\right)\left(f \prod_{u=1}^{a-1}\left(t_{a}^{2}-t_{u}^{2}\right)^{-1}\left(2 t_{a}\right)^{-1}\right) .
\end{aligned}
$$

Let us compute $\tilde{\partial}_{[1, a]}\left(t_{a}^{k}\right)$. Note that $\left(1+\gamma_{a}\right)\left(t_{a}^{k}\right)=2 t_{a}^{k}$ if $k$ is even, and zero otherwise. Hence $\tilde{\partial}_{[1, a]}\left(t_{a}^{k}\right)$ is nontrivial only when $k$ is odd, and in this case by Example (b)

$$
\begin{aligned}
\tilde{\partial}_{[1, a]}\left(t_{a}^{k}\right) & =\sum_{p=1}^{a} s_{(p, a)}\left(t_{a}^{k-1} \prod_{u=1}^{a-1}\left(t_{a}^{2}-t_{u}^{2}\right)^{-1}\right) \\
& =h_{(k-1) / 2-a+1,[1, a]}^{\jmath} .
\end{aligned}
$$


The Casimir element is given by

$$
\tilde{\pi}_{[1, a]}=\left(t_{a} \otimes 1+1 \otimes t_{a}\right) \sum_{r=0}^{b-a} t_{a}^{2(a-1-r)} \otimes(-1)^{r} e_{r,[1, a-1]}^{\jmath} .
$$

5.4. Action of $\mathfrak{U}^{\jmath}$ on the Schur 2-category. Recall Khovanov and Lauda defined a 2-functor $\Gamma^{\mathfrak{a}}: \mathfrak{U} \rightarrow \mathfrak{F}^{\mathfrak{a}}$ in [KL10], giving a 2-representation in terms of (equivariant) cohomology rings of partial flag varieties of type A. We now define an analogous 2-functor

$$
\Gamma: \mathfrak{U}^{\jmath} \longrightarrow \mathfrak{F}_{r, m}
$$

On objects $\Gamma$ is given by

$$
\mathrm{X}_{\jmath} \ni \lambda \mapsto \begin{cases}I_{\mathbf{a}} & \text { if } \lambda=\lambda(\mathbf{a}) \\ 0 & \text { otherwise }\end{cases}
$$

On the generating 1-morphisms $\Gamma$ sends

$$
\begin{aligned}
1_{\lambda}\{s\} & \mapsto \begin{cases}R^{\mathbf{a}}\{s\} & \text { if } \lambda=\lambda(\mathbf{a}), \\
0 & \text { otherwise, }\end{cases} \\
\mathcal{E}_{i} 1_{\lambda}\{s\} & \mapsto \begin{cases}R^{\mathbf{a}^{+i}}\left\{s+1+a_{i}-a_{i+1}\right\} & \text { if } \lambda=\lambda(\mathbf{a}), \\
0 & \text { otherwise, }\end{cases} \\
\mathcal{F}_{i} 1_{\lambda}\{s\} & \mapsto \begin{cases}R^{\mathbf{a}^{-i}}\left\{s+1+a_{i-1}-a_{i}\right\} & \text { if } \lambda=\lambda(\mathbf{a}), \\
0 & \text { otherwise } .\end{cases}
\end{aligned}
$$

On the generating 2-morphisms $\Gamma$ is given as follows:

- If $\mathcal{M}$ is a monomial in only $\mathcal{E}_{i}$ 's or only $\mathcal{F}_{i}$ 's, then the multiplication map identifies the bimodules $\Gamma(\mathcal{M})$ with a subring of $R$. Thus we will write the image of $\Gamma$ on diagrams consisting of only upward arrows or only downward arrows in terms of endomorphism of $R$ which preserves the corresponding subrings. Then

$$
\Gamma(\underset{i}{\uparrow} \lambda)=t_{a_{i}+1}, \quad \Gamma(\underset{\substack{\downarrow \\ i}}{\lambda})=t_{a_{i}}
$$

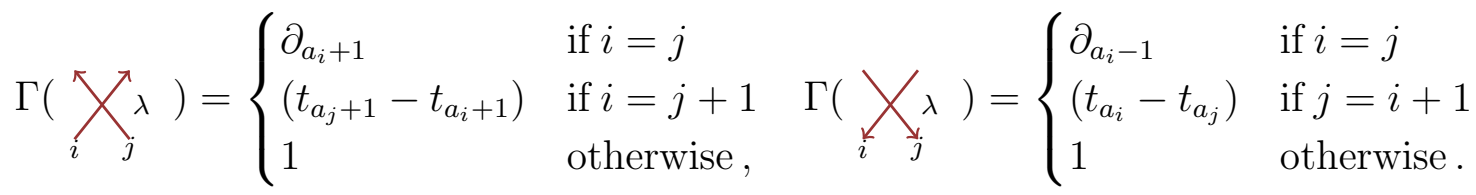

- The adjunction maps are given by

$$
\begin{aligned}
\Gamma\left(\bigcap_{i}^{\lambda}\right): & R^{\mathbf{a}^{+i}} \otimes_{R^{+i} \mathbf{a}} R^{\mathbf{a}^{+i}}\left\{1-\delta_{i, \diamond}+a_{i-1}-a_{i+1}\right\} \longrightarrow R^{\mathbf{a}}\left\{1-\left\langle{ }^{\theta} \alpha_{i}^{\vee}, \lambda+\alpha_{i}\right\rangle\right\}, \\
& f \otimes g \mapsto \partial_{\left[a_{i+1}, a_{i}+1\right]}(f g),
\end{aligned}
$$




$$
\begin{aligned}
& \Gamma\left(\bigcup_{\lambda}^{i}\right): \quad R^{\mathbf{a}} \longrightarrow R^{\mathbf{a}^{-i}} \otimes_{R^{-i} \mathbf{a}} R^{\mathbf{a}^{-i}}\left\{a_{i-1}-a_{i+1}+\left\langle{ }^{\theta} \alpha_{i}^{\vee}, \lambda\right\rangle\right\}, \\
& 1 \mapsto \pi_{\left[a_{i+1}, a_{i}\right]} \\
& \Gamma\left(\bigcap_{i}^{\lambda}\right): \quad R^{\mathbf{a}^{-i}} \otimes_{R^{-i} \mathbf{a}} R^{\mathbf{a}^{-i}}\left\{1+a_{i-1}-a_{i+1}\right\} \longrightarrow R^{\mathbf{a}}\left\{-1+\left\langle{ }^{\theta} \alpha_{i}^{\vee}, \lambda\right\rangle\right\}, \\
& f \otimes g \mapsto \begin{cases}\tilde{\partial}_{\left[1, a_{i}\right]}(f g), & \text { if } i=\diamond, \\
\partial_{\left[a_{i-1}+1, a_{i}\right]}(f g), & \text { otherwise }\end{cases} \\
& \Gamma\left(\bigcup_{\lambda}^{i}\right): \quad R^{\mathbf{a}} \longrightarrow R^{\mathbf{a}^{+i}} \otimes_{R^{+i}} \mathbf{a} R^{\mathbf{a}^{+i}}\left\{2-\delta_{i, \diamond}+a_{i-1}-a_{i+1}-\left\langle{ }^{\theta} \alpha_{i}^{\vee}, \lambda+\alpha_{i}\right\rangle\right\}, \\
& 1 \mapsto \begin{cases}\tilde{\pi}_{\left[1, a_{i}+1\right]} & \text { if } i=\diamond, \\
\pi_{\left[a_{i-1}+1, a_{i}+1\right]} & \text { otherwise. }\end{cases}
\end{aligned}
$$

Theorem 5.8. The above assignments define a locally essentially surjective 2-functor

$$
\Gamma: \mathfrak{U}^{\mathcal{J}} \longrightarrow \mathfrak{F}_{r, m}
$$

Proof. Obviously $\Gamma$ induces $\kappa^{-1} \circ \gamma$ on the Grothendieck group; see Proposition 5.3 and the paragraph above it for the notation. Hence it is enough to check it is well defined on 2morphisms.

First, let us check the compatibility with the grading. This is obvious on the generators $x$ and $\tau$. For $\bigcap^{\lambda}$, note that the coefficient $a_{i-1}$ for ${ }_{+i} \mathbf{a}$ is the same as the one for $\mathbf{a}$ if $i \neq \diamond$, but it differs from the one for a by -1 for $i=\diamond$. This is why $-\delta_{i, 0}$ appears in the degree shift on the left. Recall that $\lambda_{i}=-a_{i-1}+2 a_{i}-a_{i+1}$ and $\left\langle{ }^{\theta} \alpha_{i}^{\vee}, \alpha_{i}\right\rangle=2+\delta_{i, \diamond}$. Hence

$$
1-\delta_{i, \diamond}+a_{i-1}-a_{i+1}-1+\left\langle{ }^{\theta} \alpha_{i}^{\vee}, \lambda+\alpha_{i}\right\rangle=2+2 a_{i}-2 a_{i+1}=\operatorname{deg}\left(\partial_{\left[a_{i+1}, a_{i}+1\right]}\right) .
$$

Using similar computations, we can verify that the other three adjunction maps are compatible with grading.

Next, let us check the relations in $\widetilde{\mathfrak{U}}^{\jmath}$. By definition, the 2-morphism generators which do not involve $i=\diamond$ act in the same way as in [KL10, hence all the relations involving only these generators are satisfied. The computations for relations (3.2)-(3.6), (3.11), (3.16), even with $i=\diamond$ involved, are also entirely similar to loc. cit. , and we omit the details. The adjunction (3.1) for $i=\diamond$ follows from the fact that $\tilde{\partial}_{\left[1, a_{i}\right]}$ is a Frobenius form, see Example 5.7(c).

To check the bubble relations and the bicross relations for $i=\diamond$, let us write $a=a_{\diamond}, b=a_{\diamond+1}$. By Example 5.7, we have

$$
\begin{aligned}
& \Gamma\left(\bigodot_{s} \lambda\right)=2 \sum_{p=0}^{a}(-1)^{p} e_{p,[1, a]}^{\jmath} h_{-\lambda_{\diamond}+1-2 p+s,[a+1, b]}, \\
& \Gamma\left(\bigodot_{s} \lambda\right)=\sum_{p=0}^{b-a}(-1)^{p} e_{p,[a+1, b]} h_{\frac{\lambda_{\diamond}+2-p+s}{2},[1, a]}^{\jmath}
\end{aligned}
$$


Let $z$ be a formal variable. Then we have

$$
\begin{aligned}
& \sum_{s \in \mathbb{Z}} \Gamma\left(\begin{array}{c}
\bigcup_{\lambda_{\diamond}} \lambda \\
\lambda_{-1+s}
\end{array}\right) z^{s}=2 \sum_{s \in \mathbb{Z}} \sum_{p \geqslant 0}(-1)^{p} e_{p,[1, a]}^{\jmath} z^{2 p} h_{s-2 p,[a+1, b]} z^{s-2 p}=\frac{2 \prod_{i=1}^{a}\left(1-t_{i}^{2} z^{2}\right)}{\prod_{i=a+1}^{b}\left(1-t_{i} z\right)}, \\
& \sum_{s \in \mathbb{Z}} \Gamma\left(\begin{array}{c}
\bigodot_{\lambda} \lambda \\
-\lambda_{\diamond}-2+s
\end{array}\right) z^{s}=\sum_{s \in \mathbb{Z}} \sum_{p \geqslant 0}(-1)^{p} e_{p,[a+1, b]} z^{p} h_{\frac{s-p}{2},[1, a]}^{\jmath} z^{s-p}=\frac{\prod_{i=a+1}^{b}\left(1-t_{i} z\right)}{\prod_{i=1}^{a}\left(1-t_{i}^{2} z^{2}\right)} .
\end{aligned}
$$

All bubble relations for $i=\diamond$ now follow from that

$$
\text { LHS }(\underline{5.15}) \cdot \operatorname{LHS}(\underline{5.16})=\operatorname{RHS}(\underline{5.15}) \cdot \operatorname{RHS}(\underline{5.16})=2 \text {. }
$$

To check the bicross relations (3.14)-(3.15), note that we have

$$
\begin{aligned}
& \Gamma(\nwarrow) \cdot(1 \otimes 1)=\Gamma(\precsim \|) \cdot\left(\sum_{s=0}^{b-a} 1 \otimes 1 \otimes t_{a}^{b-a-s} \otimes(-1)^{s} e_{s,[a+1, b]}\right) \\
& =\Gamma(\bigcap \uparrow \mid) \cdot\left(\sum_{s=0}^{b-a-1} \sum_{k=0}^{b-a-s-1} 1 \otimes t_{a+1}^{b-a-s-k-1} \otimes t_{a}^{k} \otimes(-1)^{s} e_{s,[a+1, b]}\right) \\
& =1 \otimes 1 \text {, } \\
& \Gamma(\searrow) \cdot(1 \otimes 1)=\Gamma(\bigsqcup) \cdot\left(\sum_{s=0}^{a}(-1)^{a-s}\left(t_{a+1} e_{s,[1, a]}^{\jmath} \otimes t_{a+1}^{2(a-s)}+e_{s,[1, a]}^{\jmath} \otimes t_{a+1}^{2(a-s)+1}\right) \otimes 1 \otimes 1\right) \\
& =\Gamma(\mid \uparrow \cap) \cdot\left(\sum _ { s = 0 } ^ { a } ( - 1 ) ^ { a - s - 1 } \left(\sum_{k=0}^{2(a-s)-1} t_{a+1} e_{s,[1, a]}^{\jmath} \otimes t_{a+1}^{2(a-s)-k-1} \otimes t_{a}^{k}\right.\right. \\
& \left.\left.+\sum_{k=0}^{2(a-s)} e_{s,[1, a]}^{\jmath} \otimes t_{a+1}^{2(a-s)-k} \otimes t_{a}^{k}\right) \otimes 1\right) \\
& =-t_{a+1} \otimes 1-1 \otimes t_{a+1} .
\end{aligned}
$$

The last equality follows by noting that only the terms with $k=2 a-1, s=0$ contribute. This shows that the relations (3.14) and (3.15) are correct on $1 \otimes 1$. On the other hand, a direct diagram computation shows that both sides of each of these relations have the same commutator with multiplication by $x \otimes 1$ or $1 \otimes x$, so the relations are correct on all elements of the form $\left(x^{p} \otimes x^{q}\right) \cdot(1 \otimes 1)=t_{a+1}^{p} \otimes t_{a+1}^{q}$.

This completes the verification of all relations of generating 2-morphisms under $\Gamma$, and so $\Gamma$ is well defined on $\widetilde{\mathfrak{U}}^{\jmath}$.

Finally, by Proposition 5.3 and (5.2), the $\mathrm{\jmath}$-Serre relations hold in the Grothendieck group, so Proposition 4.10 implies that this representation of $\widetilde{\mathfrak{U}}^{\mathfrak{j}}$ factors through $\mathfrak{U}^{\mathfrak{J}}$. We are done.

Remark 5.9. A cyclic version of $\mathfrak{U}$ was introduced in [BHLW]. There is also an analogue of the cyclic version for $\mathfrak{U}^{\jmath}$, and let us denote it by $\mathfrak{U}^{\text {,cyc }}$. In the definition of $\Gamma$, if we use adjunctions defined by Demazure operators $\partial_{w_{I, I^{\prime}}}$ in Example 5.7 instead of $\partial_{[a, b]}$, then the same formulas define a 2 -functor $\mathfrak{U}^{\jmath, \text { cyc }} \rightarrow \mathfrak{F}_{r, m}$; we will not use this version in this paper. 
5.5. Decategorification. A 2-representation of a 2-category $\mathfrak{C}$ is a 2 -functor from $\mathfrak{C}$ to the 2 -category of $\mathbf{k}$-linear categories. That is to each object of $\mathfrak{C}$, we associate a graded $\mathbf{k}$-linear category, to each 1-morphisms a functor between corresponding categories, and 2-morphisms are sent to natural transformation of functors. The 2-category $\mathfrak{F}_{r, m}$ has a 2-representation given by sending a to $R^{\mathbf{a}}$-proj for $\mathbf{a} \in \Sigma_{r, m}$ and the 1-morphisms sent to the functors

$$
\mathscr{E}_{i}=R^{\mathbf{a}^{+i}} \otimes_{R^{\mathbf{a}}}-: R^{\mathbf{a}} \text {-proj } \longrightarrow R^{+i \mathbf{a}}-\text { proj, } \quad \mathscr{F}_{i}=R^{\mathbf{a}^{-i}} \otimes_{R^{\mathbf{a}}}-: R^{\mathbf{a}}-\text { proj } \longrightarrow R^{-i \mathbf{a}}-\text { proj } .
$$

Combining it with $\Gamma$ defines a 2-representation of $\mathfrak{U}^{\jmath}$ on $\bigoplus_{\mathbf{a} \in \Sigma_{r, m}} R^{\mathbf{a}}$-proj.

Since $R^{\mathbf{a}}$ is a polynomial ring, all projective modules over it are free, so $K_{0}\left(R^{\mathbf{a}}\right.$-proj $) \cong \mathcal{A}$ for all a. We will identify this with the constant $\mathcal{A}$-valued functions on the set of the $\mathbb{F}_{\mathbf{q}^{2}}$ points of $\mathrm{G} / \mathrm{P}_{\mathbf{a}}$; these have a natural action of the $\jmath$ Schur algebra $\mathbf{S}_{r, m}^{\jmath}$ via convolution (and hence of $\mathbf{U}^{\jmath}$ by the pullback of $\gamma(\underline{5.2}))$. Let ${ }_{\mathcal{A}} \mathbf{L}(m)$ denote $\oplus_{\mathbf{a} \in \Sigma_{r, m}} K_{0}\left(R^{\mathbf{a}}\right.$-proj), which we have identified with locally constant $\mathcal{A}$-valued functions on $\cup_{\mathbf{a}} \mathrm{G} / \mathrm{P}_{\mathbf{a}}$.

Under the duality of [BKLW], if we identify Schubert constant functions on $\cup_{\mathbf{a}} \mathrm{G} / \mathrm{P}_{\mathbf{a}}$ as the tensor product $\mathbf{V}^{\otimes m}$, then this space has mutually commuting actions of $\mathbf{U}^{\jmath}$ and the Hecke algebra $\mathbf{H}_{W}$. The subspace ${ }_{\mathcal{A}} \mathbf{L}(m) \subset \mathbf{V}^{\otimes m}$ is precisely the invariants of $\mathbf{H}_{W}$; if we set $q=1$, then $\left.\mathbf{L}(m)\right|_{q=1}=\operatorname{Sym}^{m}(\mathbf{L}(1))$.

\section{Categorification And CANonical Bases}

In this section we will show that a slightly extended version of the 2 -functor $\Gamma: \mathfrak{U}^{J} \longrightarrow \mathfrak{F}_{r, m}$ is locally full. This leads to the completion of the proof of the main theorem that the 2-category $\dot{\mathfrak{U}}^{\jmath}$ categorifies ${ }_{\mathcal{A}} \mathbf{U}^{\jmath}$. Moreover, the natural projection from ${ }_{\mathcal{A}} \mathbf{U}^{\jmath}$ to the $\jmath$ Schur algebra sends canonical basis elements to canonical basis elements or to 0.

6.1. Local fullness of $\Gamma$. Following the idea of [W15b, Theorem 9, Proof \#2], we prove the theorem below.

Theorem 6.1. If $r \geqslant m$, the 2-functor $\Gamma: \mathfrak{U}_{r}^{\mathfrak{J}} \longrightarrow \mathfrak{F}_{r, m}$ is locally full.

Proof. We must prove that for any $\lambda, \mu \in \mathrm{X}$, the functor

$$
\mathcal{H o m}_{\mathfrak{U} \jmath}(\lambda, \mu) \longrightarrow \mathcal{H o m}_{\mathfrak{F}_{r, m}}(\Gamma(\lambda), \Gamma(\mu))
$$

is full, that is, it is surjective on morphisms. We may assume $\lambda=\lambda(\mathbf{a}), \mu=\lambda(\mathbf{b})$ for some $\mathbf{a}, \mathbf{b} \in \Sigma_{r, m}$, otherwise the statement is trivial. By definition, the right hand side is a full subcategory of the category of $R^{\mathbf{b}}$-gmod- $R^{\mathbf{a}}$. So it is enough to prove the following (here we recall notation $\mathrm{Hom}^{\bullet}$ from Section 2.1).

Claim ( $\left(\right.$ ). For any $M, N \in \mathcal{H} \operatorname{Hom}_{\mathfrak{U} \mathfrak{j}}(\lambda, \mu)$ monomials in $\mathcal{E}_{i}, \mathcal{F}_{i}$, the map

$$
\Gamma_{M, N}: \operatorname{Hom}_{\mathfrak{U} j}^{\bullet}(M, N) \longrightarrow \operatorname{Hom}_{R^{\mathbf{b}}-\operatorname{gmod}-R^{\mathbf{a}}}^{\bullet}(\Gamma(M), \Gamma(N))
$$

induced by $\Gamma$ is surjective.

The proof will be carried out in two steps. Set

$$
\mathbf{o}=(0,1,2, \ldots, m-1, m, m, \ldots) \in \Sigma_{r, m} .
$$

Step 1. The case $\mathbf{a}=\mathbf{b}=\mathbf{o}$. 
In this case, we are considering the category of (nonsingular) Soergel bimodules, since $I_{\mathbf{o}}=\emptyset$ and $R^{\mathbf{o}}=R$. Let

$$
\mathcal{B}_{s_{a}}=\Gamma\left(\mathcal{F}_{\diamond+a} \mathcal{E}_{\diamond+a}\right) 1_{\mathbf{o}}=R \otimes_{R^{s a}} R\{-1\} \text { for } 0 \leqslant a \leqslant m-1 .
$$

Such bimodules are called Bott-Samelson bimodules. They are generators for the 1-morphisms $\mathbf{o} \rightarrow \mathbf{o}$, since they generate the tensor category of Soergel bimodules.

Next, recall the 2-category $\mathbf{S} \dot{\mathfrak{U}}^{j}$ from Remark 5.5, by definition the 2-functor $\dot{\mathfrak{U}}^{j} \rightarrow \mathbf{S} \dot{\mathfrak{U}}^{j}$ is full, and $\Gamma$ factorises through this quotient. Hence it is enough to show $(\star)$ for 1-morphisms $M, N$ in $\mathbf{S} \dot{\mathcal{U}}$. But $K_{0}(\mathbf{S} \dot{\mathfrak{U}}(\lambda(\mathbf{o}), \lambda(\mathbf{o})))$ is the same as $\mathbf{S}_{W}(\mathbf{o}, \mathbf{o})$ by (5.3) . Since the classes $\left[\mathcal{B}_{s_{a}}\right]$ generate the algebra $\mathbf{S}_{W}(\mathbf{o}, \mathbf{o})$, the 1-morphisms $\mathcal{F}_{\diamond+a} \mathcal{E}_{\diamond+a}$ for $0 \leqslant a \leqslant m-1$ also generate the 1-morphisms in $\mathbf{S} \dot{\mathfrak{U}}(\lambda(\mathbf{o}), \lambda(\mathbf{o}))$. Thus it suffices to show $(\star)$ for all $M, N$ that are tensor products of $\mathcal{F}_{\diamond+a} \mathcal{E}_{\diamond+a}$ 's.

The category of Bott-Samelson bimodules has a description due to Elias and Williamson [EW13], using Soergel calculus, via a set of generators and relations for homomorphisms between them. We will not need the full power of this presentation, just that it gives us a small set of generators for all morphisms; this was shown earlier by Libedinsky using his light leaf basis Lib08. Given a monoidal category $\mathcal{C}$ and a collection $C$ of objects which is closed under tensor product, we say that morphisms between these objects are locally generated by a finite collection $F$ of morphisms if there is no proper subset of morphisms between the objects in $C$ which contains $F$, and the identity on each object, and is closed under composition and tensor product.

Proposition 6.2 ([Lib08, 5.1], [EW13, 6.28]). The morphisms between Bott-Samelson bimodules are locally generated by (for $s, t \in S$ ):

(a) all polynomial multiplications on the left and right;

(b) the unit, counit, multiplication and comultiplication for the Frobenius extension $R \supset R^{s}$;

(c) the unique nonzero (up to scalar) degree 0 morphism

$$
b: \underbrace{\mathcal{B}_{s} \otimes_{R} \mathcal{B}_{t} \otimes_{R} \mathcal{B}_{s} \cdots}_{m_{s t}} \longrightarrow \underbrace{\mathcal{B}_{t} \otimes_{R} \mathcal{B}_{s} \otimes_{R} \mathcal{B}_{t} \cdots}_{m_{s t}}
$$

where $m_{\text {st }}$ denotes the order of st in the Weyl group.

Thus, in order to complete Step 1, we must find 2-morphisms in $\dot{\mathfrak{U}}^{3}$ whose images are the morphisms listed in Proposition 6.2. Let us consider these in turn:

(a) By the equations (5.13)-(5.14), we see that the degree 1 bubbles with label $\diamond$ give $\pm t_{1}$. Those with label $a+\diamond$ give $\pm t_{a} \mp t_{a+1}$ by [KL10, (6.23)], for $1 \leq a \leq m-1$. Together, these generate all elements of $R$ acting on the identity 1-morphism of $\mathbf{o}$.

(b) The adjoints in $\mathfrak{U}^{\mathfrak{J}}$ are sent under $\Gamma$ to the adjoints induced by the standard Frobenius structure for $R \supset R^{s}$ by the definitions (5.9)-(15.12).

(c) Fix $i, j \in \mathbb{I}^{\jmath}$ and let $a=i-\diamond$ and $b=j-\diamond$. Let $s=s_{a}$ and $t=s_{b}$. If $|i-j|>1$, then we simply need an isomorphism $\mathcal{B}_{s_{a}} \mathcal{B}_{s_{b}} \cong \mathcal{B}_{s_{b}} \mathcal{B}_{s_{a}}$, which is supplied by the following mutual inverse diagrams:
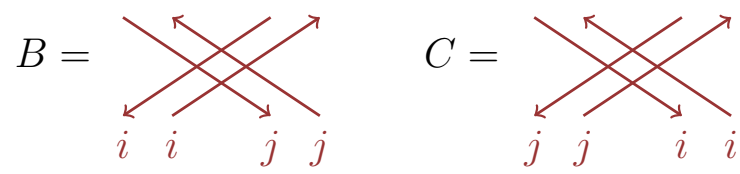
If $j=i+1$ with $i>\diamond$, then $\mathrm{b}$ (up to a scalar multiple) is given by the diagrams
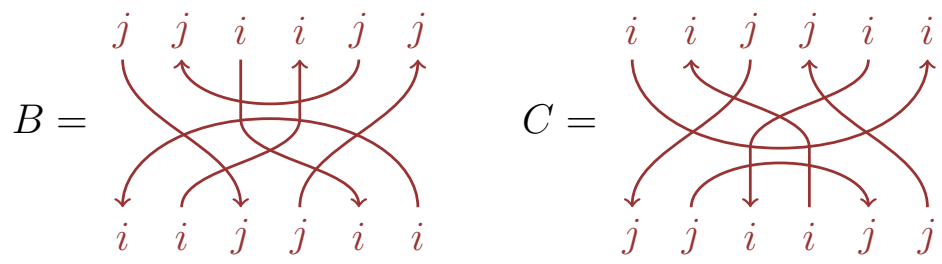

If $i=\diamond$ and $j=\diamond+1$, then $\mathrm{b}$ (up to a scalar multiple) is given by the diagrams
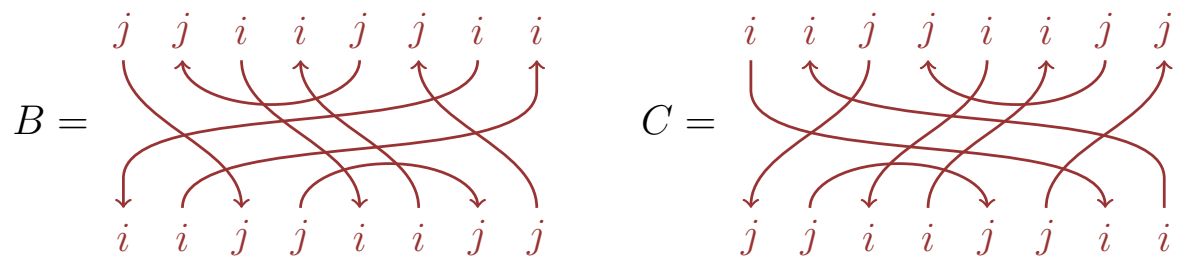

The confirmation that the morphisms of (6.2) act correctly is straightforward from the definition. The case of (6.3) is confirmed by [MSV13, 6.8]. Of course, we could verify (6.4) by direct calculation, but in fact, we have arrived at this formula systematically by applying the results of Elias [E13] on Soergel bimodules for dihedral groups. This gives a formula [E13, 6.4] for writing the degree 0 morphism in terms of the Frobenius square $R \supset R^{s}, R^{t} \supset R^{s, t}$. This square gives four Frobenius extensions:

$$
A=R, A^{\prime}=R^{s} ; \quad A=R, A^{\prime}=R^{t} ; \quad A=R^{s}, A^{\prime}=R^{s, t} ; \quad A=R^{t}, A^{\prime}=R^{s, t} .
$$

Elias's formula allows us to write the desired morphism in terms of:

(i) all polynomial multiplications on the left and right;

(ii) the unit, counit, multiplication and comultiplication for the Frobenius extensions $A \supset A^{\prime}$ listed in (6.5);

(iii) the obvious isomorphism of $\left(R^{s, t}, R\right)$-bimodules

$$
R^{s, t} R^{s, t} \otimes_{R^{s, t}} R^{s} \otimes_{R^{s}} R_{R} \cong R^{s, t} R_{R} \cong{ }_{R^{s, t}} R^{s, t} \otimes_{R^{s, t}} R^{t} \otimes_{R^{t}} R_{R} .
$$

Here and below, the left and right subscripts indicate the bimodule structure. For example, $R^{s, t} R_{R}$ stands for $R$ viewed as an $\left(R^{s, t}, R\right)$-bimodule. In order to describe these 2-morphisms in terms of the category $\mathfrak{U}^{\mathfrak{J}}$, we need to consider some auxiliary objects:

- $\mathbf{o}_{a}=(0,1, \ldots, a-1, a+1, a+1, a+2, a+3 \ldots)$ satisfies $I_{\mathbf{o}_{a}}=\left\{s_{a}\right\}$ and $R^{\mathbf{o}_{a}}=R^{s_{a}}$.

- $\mathbf{o}_{a, a+1}=(0,1, \ldots, a-1, a+2, a+2, a+2, a+3, \ldots)$ satisfies $I_{\mathbf{o}_{a}}=\left\{s_{a}, s_{a+1}\right\}$ and $R^{\mathbf{o}_{a}}=R^{s_{a}, s_{a+1}}$.

Furthermore, recalling $a=i-\diamond$ and $b=j-\diamond$, we have bimodule isomorphisms (ignoring grading shifts):

$$
\begin{aligned}
& 1_{\mathbf{o}_{a}} \mathcal{E}_{i} 1_{\mathbf{o}} \cong{ }_{R^{s a}} R_{R}, \quad 1_{\mathbf{o}} \mathcal{F}_{i} 1_{\mathbf{o}_{a}} \cong{ }_{R} R_{R^{s a}}, \\
& 1_{\mathbf{o}_{a, b}} \mathcal{E}_{i} \mathcal{E}_{j} 1_{\mathbf{o}_{a}} \cong R^{s_{a}, s_{b}} R^{s_{a}} R^{s_{a}}, \quad 1_{\mathbf{o}_{a, b}} \mathcal{E}_{i}^{(2)} 1_{\mathbf{o}_{b}} \cong R^{s_{a}, s_{b}} R^{s_{b}} R^{s_{b}}, \\
& 1_{\mathbf{o}_{a}} \mathcal{F}_{j} \mathcal{F}_{i} 1_{\mathbf{o}_{a, b}} \cong{ }_{R^{s_{a}}} R^{s_{a}}{ }_{R^{s_{a}, s_{b}}}, \quad 1_{\mathbf{o}_{b}} \mathcal{F}_{i}^{(2)} 1_{\mathbf{o}_{a, b}} \cong{ }_{R^{s_{b}}} R^{s_{b}} R^{s_{a}, s_{b}} .
\end{aligned}
$$

In order to apply Elias's formula, we must find a 2-morphism which induces an isomorphism $\mathcal{E}_{i} \mathcal{E}_{j} \mathcal{E}_{i} 1_{\mathbf{o}} \cong \mathcal{E}_{i}^{(2)} \mathcal{E}_{j} 1_{\mathbf{o}}$, since this will give (iii). Note that $\mathcal{E}_{j} \mathcal{E}_{i}^{(2)} 1_{\mathbf{o}}=0$, since the flag variety 
corresponding to $(0,1, \ldots, a+2, a+1, a+2, \ldots)$ is empty. The equation (3.5) shows that the 2-morphisms

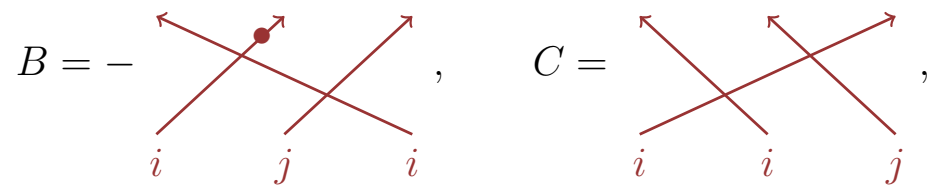

are inverse to each other and thus give the desired isomorphism. Substituting these into E13, Notation 6.4] gives the equations (6.3) and (6.4). Hence Claim ( $\star$ ) in the case of Step 1 is proved.

Step 2. The general case.

Let us first assume $\mathbf{b}=\mathbf{o}$ and $\mathbf{a}$ is arbitrary. First, assume that we have found an object $P \in \mathfrak{U}^{\jmath}(\lambda(\mathbf{o}), \lambda(\mathbf{a}))$ such that $\Gamma(P)={ }_{\mathbf{a}} R$ and such that for $Q \in \mathfrak{U}^{\jmath}(\lambda(\mathbf{a}), \lambda(\mathbf{o}))$ the adjoint of $P$, the product $P Q$ has $1_{\lambda(\mathbf{a})}$ as a direct factor in degree zero. Let $\eta: 1_{\lambda(\mathbf{a})} \hookrightarrow P Q$ be a split embedding. Then $\Gamma$ sends it to a split embedding $R^{\mathbf{a}} \cong \Gamma\left(1_{\lambda(\mathbf{a})}\right) \rightarrow R \cong \Gamma(P Q)$, with the latter viewed as a $\left(R^{\mathbf{a}}, R^{\mathbf{a}}\right)$-bimodule. We have a commutative diagram

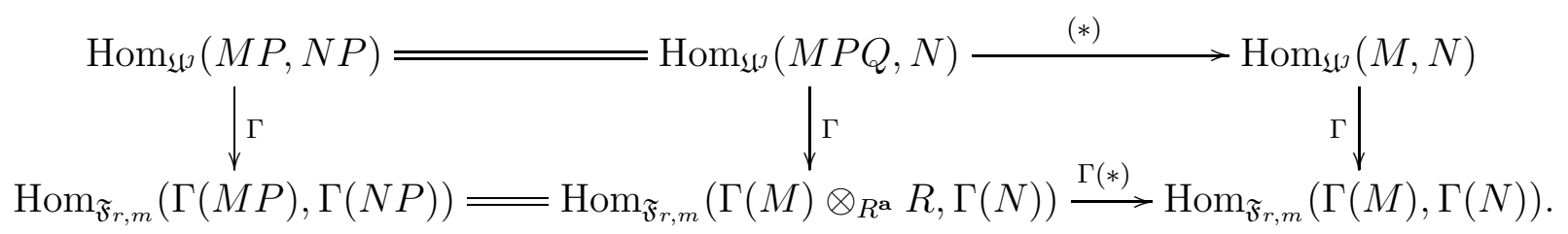

Here $(*)$ is the transpose of the map $M \eta: M \rightarrow M P Q$. It is surjective, so is its image by $\Gamma$. Since $M P$ and $N P$ belong to $\mathfrak{U}^{J}(\mathbf{o}, \mathbf{o})$, Step 1 implies that the leftmost vertical map is surjective. Hence, the rightmost vertical map is surjective too. The case for arbitrary $\mathbf{b}$ follows from the same argument by considering $\operatorname{Hom}_{\mathfrak{U} j}(Q M P, Q N P)$ on the left top corner for the $Q$ defined for $\mathbf{b}$.

It remains to find $P$. Without loss of generality, we may assume

$$
a_{\diamond}<a_{\diamond+1}<\cdots<a_{\diamond+s}=a_{\diamond+s+1}=\cdots=m, \quad \text { for some } p .
$$

Indeed, if there is any index $p$ such that $a_{\diamond+p-1}=a_{\diamond+p}<a_{\diamond+p+1}$, then let $\mathbf{a}^{\prime}$ be the sequence with $a_{\diamond+p}^{\prime}=a_{\diamond+p+1}$ and $a_{i}^{\prime}=a_{i}$ for $i \neq \diamond+p+1$. We have a split surjection $\mathcal{F}_{\diamond+p}^{\left(a_{\diamond+p+1}-a_{\diamond+p}\right)} 1_{\lambda\left(\mathbf{a}^{\prime}\right)} \mathcal{E}_{\diamond+p}^{\left(a_{\diamond+p+1}-a_{\diamond+p}\right)} 1_{\lambda(\mathbf{a})} \rightarrow 1_{\lambda(\mathbf{a})}$, and hence we get the following commutative diagram

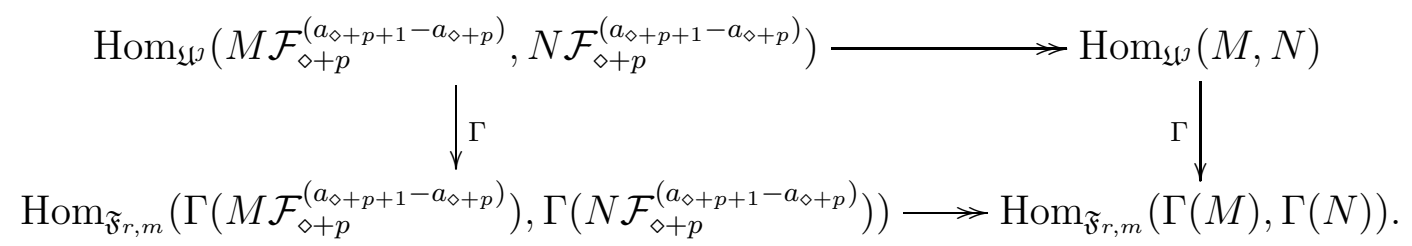

The surjectivity of the left vertical arrow implies the surjectivity of the right one. Hence, up to replacing a by $\mathbf{a}^{\prime}$ inductively, we are reduced to consider the sequence of the form (6.7).

For such a, we have $k \leqslant a_{\diamond+k}$ for all $k$. We choose a sequence $\mathbf{i}=\left(i_{1}, i_{2}, \ldots, i_{n}\right)$ such that the weight $\lambda^{(k)}=\lambda(\mathbf{o})+\alpha_{i_{1}}+\ldots+\alpha_{i_{k}}$ satisfies $\lambda^{(n)}=\lambda(\mathbf{a})$ and $\left\langle{ }^{\theta} \alpha_{i_{k}}^{\vee}, \lambda^{(k)}\right\rangle>0$ for all $k>0$. To do 
so, we proceed inductively: set $\mathbf{a}^{(0)}=\mathbf{o}$ and define $\mathbf{a}^{(k)}={ }_{+i_{k}} \mathbf{a}^{(k-1)}$ for all $k>0$. Let $i_{1}$ be the largest element such that $i_{1}-\diamond<a_{i_{1}}$. Given $i_{k}$, we then

- If the set $\left\{j<i_{k} \mid a_{j}^{(k)}<a_{j}\right\}$ is non-empty, then we let $i_{k+1}$ be the maximal element in this set.

- Otherwise, we let $i_{k+1}$ be the largest element of $\left\{j \geq i_{k} \mid a_{j}^{(k)}<a_{j}\right\}$.

Then for each $k$, we always have $a_{i_{k}+1}^{(k)}-a_{i_{k}}^{(k)}=1$ and $a_{i_{k}}^{(k)}-a_{i_{k}-1}^{(k)} \leqslant 2$. For $i_{k}=\diamond$ this inequality follows from the fact that $a_{i_{k}}^{(k)} \geqslant 1$. Thus, we have that $\left\langle{ }^{\theta} \alpha_{i_{k}}^{\vee}, \lambda^{(k)}\right\rangle \geqslant 1$ for all $k$. We then define the mutually adjoint 1-morphisms:

$$
P=1_{\lambda(\mathbf{a})} \mathcal{E}_{i_{n}} \ldots 1_{\lambda^{(2)}} \mathcal{E}_{i_{2}} 1_{\lambda^{(1)}} \mathcal{E}_{i_{1}} 1_{\lambda(\mathbf{o})} \quad Q=1_{\lambda(\mathbf{o})} \mathcal{F}_{i_{1}} 1_{\lambda^{(1)}} \mathcal{F}_{i_{2}} 1_{\lambda^{(2)}} \ldots \mathcal{F}_{i_{n}} 1_{\lambda(\mathbf{a})} .
$$

Since $\left\langle{ }^{\theta} \alpha_{i_{k}}^{\vee}, \lambda^{(k)}\right\rangle \geqslant 1$ for all $k$, it follows from (3.7) and (3.9) that

$$
\overbrace{i_{k}}^{\lambda}{\left.\stackrel{1}{ }{ }^{\theta} \alpha_{i_{k}}^{\vee}, \lambda^{(k)}\right\rangle-1}^{(k)} \mathcal{E}_{i} \mathcal{F}_{i} 1_{\lambda} \longrightarrow 1_{\lambda}
$$

is split surjective. Hence $1_{\lambda^{(k)}}$ is a direct factor of $\mathcal{E}_{i_{k}} \mathcal{F}_{i_{k}} 1_{\lambda^{(k)}}$ of degree zero. Applying this successively, we obtain that $1_{\lambda(\mathbf{a})}$ is a direct factor of $P Q$ of degree zero.

This completes the proof of Claim $(\star)$ and hence the proof of Theorem 6.1.

For an arbitrary $r$, there is an obvious problem with extending Theorem 6.1; the images of the bubbles under $\Gamma$ do not generate $R^{\mathbf{a}}$ unless $a_{i}=a_{i+1}$ for some $i$ or $a_{\diamond}=0$. We are able to make the proof work for $r \geq m$, since one of these conditions will be forced by the pigeonhole principle, but for $r<m$, the obvious extension of Theorem 6.1 fails. However, this failure to surject to $R^{\mathbf{a}}$ is easily fixed, and it proves to be the only obstruction to fullness.

Fixing this issue requires us to add more 2-morphisms to $\mathfrak{U}_{r}^{\mathfrak{J}}$, analogous to the extension of $\mathfrak{U}$ discussed in [W15b, Section 2.1]. Let us write $\mathfrak{U}^{\jmath}, \Gamma$ and $X_{\jmath}$ as $\mathfrak{U}_{r}^{\jmath}, \Gamma^{r}$ and $X_{\jmath, r}$ to indicate the dependence on rank $r$. Note that for $r<s$, there is a natural embedding $\mathrm{X}_{\jmath, r} \rightarrow \mathrm{X}_{\jmath, s}$ given by sending the class of $\sum_{i=-r}^{r} k_{i} \varepsilon_{i}$ to the class denoted by the same notation in $\mathrm{X}_{\jmath, s}$. We have a well-defined 2-functor

$$
\iota_{r, s}: \mathfrak{U}_{r}^{\jmath} \longrightarrow \mathfrak{U}_{s}^{J}
$$

which is given by the above embedding on objects, sending $\mathcal{E}_{i}, \mathcal{F}_{i}$ to themselves for $i \in \mathbb{I}_{r}^{\jmath}$, and sending the generators of 2-morphisms in $\mathfrak{U}_{r}^{\jmath}$ to the same diagram in $\mathfrak{U}_{s}^{\jmath}$. Now, for each $\lambda \in \mathrm{X}_{\jmath, r}$, consider the ring $Z_{\lambda}^{(s)}=\operatorname{End}_{\mathfrak{U}_{s}^{\jmath}}\left(1_{\lambda}\right)$. Using arguments as in [KL10, Proposition 3.6], we can see that $Z_{\lambda}^{(s)}$ is generated by bubbles indexed by $i \in \mathbb{I}_{s}^{3}$, and that for any pair of 1-morphisms $M, N \in \mathfrak{U}_{r}^{\jmath}$, the space $\operatorname{Hom}_{\mathfrak{U}_{s}^{\mathfrak{j}}}^{\bullet}(M, N)$ is generated by the image $\iota_{r, s}\left(\operatorname{Hom}_{\mathfrak{U}_{r}^{j}}^{\bullet}(M, N)\right)$ and $Z_{\lambda}^{(s)}$. We define $\mathfrak{U}_{r}^{j}$,ext as the full 2-subcategory of $\mathfrak{U}_{r+1}^{\mathfrak{J}}$ generated by objects and 1-morphisms in the image of $\iota_{r, r+1}$.

Next, consider the embedding $j_{r, s}: \Sigma_{r, m} \hookrightarrow \Sigma_{s, m}$ which sends $\mathbf{a}=\left(a_{1}, \ldots, a_{r}\right)$ to $j_{r, s}(\mathbf{a})=$ $\left(a_{1}, \ldots, a_{r}, m, m, \ldots\right)$. This induces a tautological 2-functor $\mathfrak{F}_{r, m} \rightarrow \mathfrak{F}_{s, m}$ which is fully faithful, since $R^{\mathbf{a}}=R^{j_{r, s}(\mathbf{a})}$. Moreover $\Gamma$ intertwines $\iota_{r, s}$ with this 2 -functor. Hence we obtain a 
commutative diagram

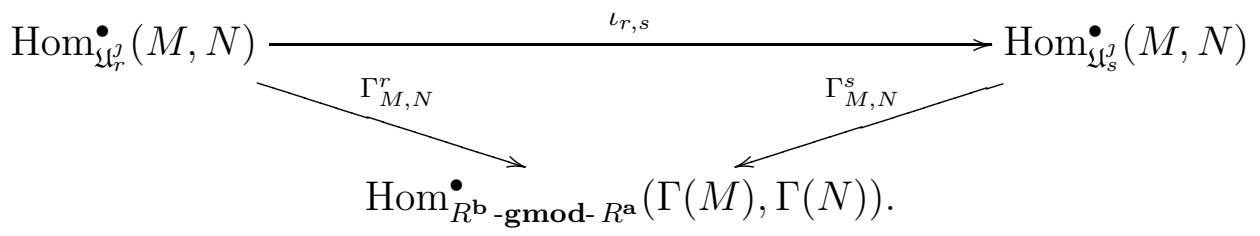

Hence $\Gamma$ extends to a 2-functor $\Gamma^{\text {ext }}: \mathfrak{U}_{r}^{\jmath, \text { ext }} \rightarrow \mathfrak{F}_{r, m}$, and we have a similar commutative diagram with $\mathfrak{U}_{r}^{\jmath}, \Gamma_{M, N}^{r}$ on the left-downward arrow replaced by their extended version. Now, if $s \geqslant m$, then the theorem implies that in the diagram above the map $\Gamma_{M, N}$ on the right is surjective. Further by formulas similar to (5.13)-(5.14), $\Gamma$ sends positive degree bubbles labeled by $i$ to 0 if $i>r+1$. Therefore we have established the following extension of Theorem 6.1.

Proposition 6.3. The 2-functor $\Gamma^{\mathrm{ext}}: \mathfrak{U}_{r}^{\jmath, \text { ext }} \rightarrow \mathfrak{F}_{r, m}$ is locally full for any $r$ and $m$.

Note that $\mathfrak{U}_{r}^{\jmath, \text { ext }}$ only differs from $\mathfrak{U}_{r}^{\jmath}$ by bubbles labeled by $\diamond+r$, which live in strictly positive degree. Hence the canonical embedding $\dot{\mathfrak{U}}_{r}^{\jmath} \rightarrow \dot{\mathfrak{U}}_{r}^{\text {,ext }}$ sends indecomposable 1-morphisms to indecomposable ones, and induces an isomorphism on Grothendieck groups (as argued in [W15b, Proposition 3]). Applying [W15b, Lemma 10], we have established the following corollary.

Corollary 6.4. The map $K_{0}\left(\dot{\mathfrak{U}}^{\mathfrak{j}}\right) \rightarrow K_{0}\left(\mathfrak{F}_{r, m}\right)$ induced by $\Gamma$ sends the classes of indecomposable 1-morphisms in $\dot{\mathfrak{U}}^{\jmath}$ which are not annihilated by $\Gamma$ bijectively to the classes of indecomposable 1-morphisms in $\mathfrak{F}_{r, m}$.

6.2. Canonical basis. Recall the functor $\aleph:{ }_{\mathcal{A}} \dot{\mathbf{U}}^{\jmath} \rightarrow K_{0}\left(\dot{\mathfrak{U}}^{\jmath}\right)$ defined in Lemma 4.6, and the equivalence $\kappa$ in Proposition 5.3. By construction, we have a commutative diagram

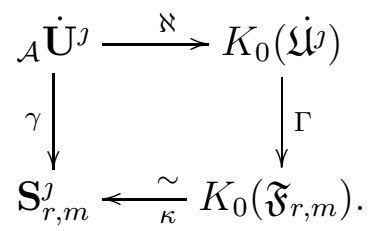

Recall also the canonical basis $\mathbf{B}^{\jmath}$ for ${ }_{\mathcal{A}} \mathbf{U}^{\jmath}$ defined in [LW15, Proposition 5.4, Theorem 5.5]. It can be characterized as the unique basis such that $\gamma$ maps each element in it to an element in the canonical basis of $\mathbf{S}_{r, m}^{\jmath}$ for infinitely many $m$.

\section{Theorem 6.5.}

(a) The functor $\aleph:{ }_{\mathcal{A}} \dot{\mathbf{U}}^{\jmath} \rightarrow K_{0}\left(\dot{\mathfrak{U}^{\jmath}}\right)$ is an equivalence of categories.

(b) Assume the residue field of $\mathbf{k}$ has characteristic zero. Then $\aleph$ sends canonical basis of ${ }_{\mathcal{A}} \dot{\mathbf{U}}^{3}$ to the classes of self-dual indecomposable 1-morphisms in $\dot{\mathfrak{U}}$.

Proof. To prove (a), note that $\aleph$ is identity on objects, and it is full by Proposition 4.8. It remains to show it is faithful, i.e., the map $\aleph_{\lambda, \mu}:{ }_{\mathcal{A}} \dot{\mathbf{U}}^{\jmath}(\lambda, \mu) \rightarrow K_{0}\left(\dot{\mathfrak{U}}^{\jmath}(\lambda, \mu)\right)$ is injective. Note that if $u$ is in the kernel of $\aleph_{\lambda, \mu}$, by the commutativity of (6.8) and the fact that $\kappa$ is invertible, we deduce that $\gamma(u)=0$ for all $m \in \mathbb{N}$. By [BKLW, Theorem 4.7], this is possible only when $u=0$. We are done. Part (b) follows from the characterization of $\mathbf{B}^{\jmath}$ and Corollary 6.4 . 
As a corollary of Theorem 6.5] and Corollary 6.4, we obtain the following refinement of [LW15, Proposition 5.11].

Corollary 6.6. The map $\gamma:{ }_{\mathcal{A}} \dot{\mathbf{U}}^{\jmath} \rightarrow \mathbf{S}_{r, m}^{\jmath}$ in (5.2) sends $\mathbf{B}^{\jmath} \backslash\left(\mathbf{B}^{\jmath} \cap\right.$ ker $\left.\gamma\right)$ bijectively to the canonical basis of $\mathbf{S}_{r, m}^{J}$ for all $m$.

\section{Categorical action on Category $\mathcal{O}$}

In this section, we assume $\mathbf{k}=\mathbb{C}$.

7.1. Reminders on Harish-Chandra bimodules. Let $\mathfrak{g}$ be a complex semisimple Lie algebra and $\mathfrak{t} \subset \mathfrak{g}$ a Cartan subalgebra. Let $U=U(\mathfrak{g})$ be the enveloping algebra of $\mathfrak{g}$ and let $Z$ be the center of $U$. We view the ring $R=S(\mathfrak{t})$ as the coordinate ring of $\mathfrak{t}^{*}$. Recall that the Harish-Chandra morphism $\xi: Z \rightarrow R$ is a ring homomorphism such that an element $z \in Z$ acts on a Verma module of highest weight $\lambda$ by the value of $\xi(z)$ at $\lambda$.

Consider the dot action of the Weyl group $\mathrm{W}$ on $\mathfrak{t}^{*}$ by $w \bullet \lambda=w(\lambda+\rho)-\rho$, where $\rho$ is the half sum of positive roots. Then $\xi$ sends $Z$ isomorphically onto $W$-invariant functions on $\mathfrak{t}^{*}$ for the dot action. Equivalently, the central characters of $Z$ are in bijection with $W$-dot-orbits in $\mathfrak{t}^{*}$. For $\lambda \in \mathfrak{t}^{*}$, denote the corresponding central character by $\chi_{\lambda}$ and write $I_{\lambda}=$ ker $\chi_{\lambda}$.

We say that $\chi_{\lambda}$ is integral if $\lambda$ is an integral weight of $\mathfrak{g}$. Let $\widehat{Z}_{\lambda}$ be the completion of $Z$ at $\lambda$. Let $\lambda^{\#}: R \rightarrow R$ be the pull back of the translation map $\mathfrak{t}^{*} \rightarrow \mathfrak{t}^{*}, x \mapsto x+\lambda$. Then $\lambda^{\#} \circ \xi: Z \rightarrow R$ induces an isomorphism $\widehat{Z}_{\lambda} \simeq \widehat{R}^{W_{\lambda}}$, where $\widehat{R}$ is the completion of $R$ at the ideal generated by $\mathfrak{t}$, and $W_{\lambda}$ is the stabilizer of $\lambda$ under the dot action. We abbreviate $\widehat{R}^{\lambda}=\widehat{R}^{W_{\lambda}}$ and denote by $\mathfrak{m}_{\lambda}$ the maximal ideal of $\widehat{R}^{W_{\lambda}}$.

Recall that a Harish-Chandra bimodule over $\mathfrak{g}$ is a $(U, U)$-bimodule for which the adjoint action of $\mathfrak{g}$ is locally finite [BG80, Soe92]. Typical examples of Harish-Chandra modules are $E \otimes U$, where $E$ is a finite dimensional left $U$-module. The left $U$-action on $E \otimes U$ is induced by the $\mathfrak{g}$-action such that $u(x \otimes y)=(u x) \otimes y+x \otimes(u y)$ for $u \in \mathfrak{g}$, and the right action is the right action of $U$ on itself. In other words, it is the tensor product of $U$ with $E$ considered as a bimodule with the usual left action and trivial right action.

Let $\mathcal{H}$ be the category of finitely generated Harish-Chandra bimodules of finite length. It is a direct sum of subcategories of the form

$$
{ }_{\lambda} \mathcal{H}_{\mu}=\left\{M \in \mathcal{H} \mid I_{\lambda}^{n} M=M I_{\mu}^{n}=0 \text { for } n \gg 0\right\}
$$

for $\lambda, \mu \in \mathfrak{t}^{*}$. Let ${ }_{\lambda} \mathcal{H}_{\mu}^{n}$ be the full subcategory of ${ }_{\lambda} \mathcal{H}_{\mu}$ consisting of modules $M$ such that $M I_{\mu}^{n}=0$. Let $\widehat{U}_{\lambda}$ be the completion of $U$ at $I_{\lambda}$. Let ${ }_{\lambda} \widehat{\mathcal{H}}_{\mu}$ denote the category of finitely generated $\left(\widehat{U}_{\lambda}, \widehat{U}_{\mu}\right)$-bimodules.

Theorem 7.1 ([Soe92, Str04]).

(a) There is a unique exact functor

$$
\mathbb{V}:{ }_{\lambda} \mathcal{H}_{\mu} \longrightarrow \widehat{R}^{\lambda} \text {-mod- } \widehat{R}^{\mu}
$$

defined by the property that it sends the unique simple module of maximal GelfandKirillov dimension to the trivial module $\mathbb{C}$, and all the other simple modules to zero. 
For each $n \geqslant 0$, it induces an exact functor

$$
\mathbb{V}:{ }_{\lambda} \mathcal{H}_{\mu}^{n} \longrightarrow \widehat{R}^{\lambda}-\bmod -\left(\widehat{R}^{\mu} / \mathfrak{m}_{\mu}^{n}\right)
$$

(b) For any $n>0$, the category ${ }_{\lambda} \mathcal{H}_{\mu}^{n}$ has enough projective objects, given by direct sums of direct summands of modules of the form $E \otimes U /\left(U I_{\mu}^{n}\right)$, where $E$ is a finite dimensional $\mathfrak{g}$-module.

(c) For any $n>0$, the functor $\mathbb{V}$ restricts to a fully faithful functor on the full additive subcategory ${ }_{\lambda} \mathcal{P}_{\mu}^{n}$ of projective objects in ${ }_{\lambda} \mathcal{H}_{\mu}^{n}$.

Proof. The definition of $\mathbb{V}$ is given in [Str04, Page 357], Part (b) is proved in [Str04, Theorem 1.1], and Part (c) follows from [Str04, Theorem 4.1].

Let $\mathcal{M}$ be the category of (left) $U$-modules $M$ over which $Z$ acts locally finitely. It is a direct sum over $\mu \in \mathfrak{t}^{*}$ of subcategories ${ }_{\mu} \mathcal{M} \subset \mathcal{M}$ consisting of modules over which $I_{\mu}$ acts locally nilpotently. Denote by $\operatorname{pr}_{\mu}: \mathcal{M} \rightarrow{ }_{\mu} \mathcal{M}$ the projection functor. Let ${ }_{\mu}^{n} \mathcal{M} \subset{ }_{\mu} \mathcal{M}$ be the full subcategory consisting of modules over which $I_{\mu}^{n}$ acts trivially.

Recall that a projective functor $\mathcal{M} \rightarrow \mathcal{M}$ is a direct summand of $E \otimes-$ for some finite dimensional $U$-module $E$. Direct sums and compositions of projective functors are again projective functors. Given $E$ a finite dimensional representation, consider the projective functor

$$
F:{ }_{\mu} \mathcal{M} \longrightarrow{ }_{\lambda} \mathcal{M}, \quad M \mapsto \operatorname{pr}_{\lambda}(E \otimes M)
$$

It is nontrivial if and only if $\lambda-\mu$ is a weight of $E$. The restriction of $F$ to the subcategory ${ }_{\mu}^{n} \mathcal{M}$ is represented by a direct factor ${ }_{\lambda} \Phi(E)_{\mu}^{n}$ of $E \otimes U / U I_{\mu}^{n}$, which is an object of ${ }_{\lambda} \mathcal{P}_{\mu}^{n}$. For $m>n$, we have a projection ${ }_{\lambda} \Phi(E)_{\mu}^{m} \rightarrow{ }_{\lambda} \Phi(E)_{\mu}^{n}={ }_{\lambda} \Phi(E)_{\mu}^{m} / I_{\mu}^{n}$. Taking the limit yields an object ${ }_{\lambda} \Phi(E)_{\mu}=\lim _{\lambda} \Phi(E)_{\mu}^{n}$ in ${ }_{\lambda} \widehat{\mathcal{H}}_{\mu}$ which represents the functor $F$. Let ${ }_{\lambda} \widehat{\mathcal{P}}_{\mu}$ be the full additive subcategory of ${ }_{\lambda} \widehat{\mathcal{H}}_{\mu}$ generated by direct summands of ${ }_{\lambda} \Phi(E)_{\mu}$ for all $E$.

Corollary 7.2. The functor $\mathbb{V}$ induces a fully faithful functor

$$
\widehat{\mathbb{V}}:{ }_{\lambda} \widehat{\mathcal{P}}_{\mu} \longrightarrow \widehat{R}^{\lambda} \text {-mod- } \widehat{R}^{\mu} \text {. }
$$

Proof. To see this, note that for any object $M$ in ${ }_{\lambda} \widehat{\mathcal{P}}_{\mu}$ we have $M=\lim M / M I_{\mu}^{n}$. Applying $\mathbb{V}$ to the natural projection $M / M I_{\mu}^{m} \rightarrow M / M I_{\mu}^{n}$ yields a surjective map $\overleftarrow{V}\left(M / M I_{\mu}^{m}\right) \rightarrow \mathbb{V}\left(M / M I_{\mu}^{n}\right)$. Define $\widehat{\mathbb{V}}(M)$ as the limit of the projective system $\left\{\mathbb{V}\left(M / M I_{\mu}^{n}\right)\right\}_{n \geq 0}$. It is still an object in $\widehat{R}^{\lambda}$-mod- $\widehat{R}^{\mu}$. We have $\widehat{\mathbb{V}}(M) / \mathfrak{m}_{\mu}^{n}=\mathbb{V}\left(M / M I_{\mu}^{n}\right)$. Finally, for two objects $M, N$ in ${ }_{\lambda} \widehat{\mathcal{P}}_{\mu}$, we have

$$
\begin{aligned}
& \operatorname{Hom}_{\lambda} \widehat{\mathcal{P}}_{\mu}(M, N)=\lim _{\longleftarrow} \operatorname{Hom}_{\hat{\mathcal{H}}}\left(M, N / N I_{\mu}^{n}\right) \\
& =\lim _{\longleftarrow} \operatorname{Hom}_{\lambda} \mathcal{H}_{\mu}^{n}\left(M / M I_{\mu}^{n}, N / N I_{\mu}^{n}\right) \\
& =\lim _{\longleftarrow} \operatorname{Hom}_{\widehat{R}^{\lambda} \otimes \widehat{R}^{\mu}}\left(\mathbb{V}\left(M / M I_{\mu}^{n}\right), \mathbb{V}\left(N / N I_{\mu}^{n}\right)\right) \\
& =\lim _{\longleftarrow} \operatorname{Hom}_{\widehat{R}^{\lambda} \otimes \widehat{R}^{\mu}}\left(\widehat{\mathbb{V}}(M) / \mathfrak{m}_{\mu}^{n}, \widehat{\mathbb{V}}(N) / \mathfrak{m}_{\mu}^{n}\right) \\
& =\operatorname{Hom}_{\widehat{R}^{\lambda} \otimes \widehat{R}^{\mu}}(\widehat{\mathbb{V}}(M), \widehat{\mathbb{V}}(N)) \text {. }
\end{aligned}
$$

where the first equality is the universal property of projective limit and the second one is because $I_{\mu}^{n} \subset Z$ is central, the third equality is given by Theorem 7.1(c). 
Lemma 7.3. For $M \in{ }_{\lambda} \widehat{\mathcal{P}}_{\mu}$ and $N \in{ }_{\mu} \widehat{\mathcal{P}}_{\nu}$ we have $M \otimes_{\widehat{U}_{\mu}} N \in{ }_{\lambda} \widehat{\mathcal{P}}_{\nu}$. Moreover there is a natural isomorphism $\widehat{V}\left(M \otimes_{\widehat{U}_{\mu}} N\right) \cong \widehat{\mathbb{V}}(M) \otimes_{\widehat{R}^{\mu}} \widehat{\mathbb{V}}(N)$, which is functorial with respect to $M$ and $N$.

Proof. The first statement follows from the fact that composition of projective functors is again a projective functor. The proof of the second statement is similar to [Soe92, Proposition 13], details are left to the reader.

7.2. The case of types $\mathbf{B}$ and $\mathbf{C}$. Now, let us apply the above results to the type B/C situation. Let $\mathfrak{g}=\mathfrak{s o}_{2 m+1}$ or $\mathfrak{g}=\mathfrak{s p}_{2 m}$. We choose $\mathbf{a}=\left(a_{\diamond}, a_{1+\diamond}, \ldots, a_{r-\diamond}\right) \in \Sigma_{r, m}$; by convention, we have $a_{r+\diamond}=m$. To this vector, we associate a weight $\mu\langle\mathbf{a}\rangle \in \mathfrak{t}^{*}$ such that

$$
\mu\langle\mathbf{a}\rangle+\rho=(0, \ldots, 0,-1, \ldots,-1, \ldots,-r, \ldots,-r)
$$

with 0 appearing $a_{\diamond}$ times, and $-k$ appearing $a_{k+\diamond}-a_{k-\diamond}$ times for $k>0$. By definition, we have that $W_{\mu}\langle\mathbf{a}\rangle$ is the subgroup of $W$ generated by the reflections in $I_{\mathbf{a}} \subset \mathrm{S}$ (see Section 5.2 for notation). Thus, we have $R^{W_{\mu\langle\mathbf{a}\rangle}}=R^{\mathfrak{a}}$. Note that if $\mathfrak{g}=\mathfrak{s p}_{2 m}$ then every integral central character is of this form for $r$ sufficiently large. For $\mathfrak{g}=\mathfrak{s o}_{2 m+1}$, we have $\rho \in \sum_{a=1}^{m}\left(\mathbb{Z}+\frac{1}{2}\right) \epsilon_{a}$, and so the highest weights for modules with central character $\chi(\mathbf{a})$ lie in $\sum_{a=1}^{m}\left(\mathbb{Z}+\frac{1}{2}\right) \epsilon_{a}$; in particular, the block of the spin representation lies in this image and the block of the trivial representation does not.

The weights of the natural representation $V$ are $\pm \epsilon_{k}$ for $k=1, \ldots, m$ for $\mathfrak{g}=\mathfrak{s p}_{2 m}$ (and 0 in addition for $\left.\mathfrak{g}=\mathfrak{s o}_{2 m+1}\right)$. Hence we have ${ }_{\lambda} \Phi(V)_{\mu\langle\mathbf{a}\rangle}$ is nontrivial precisely when $\lambda=\mu\left\langle_{ \pm i} \mathbf{a}\right\rangle$ for some $i \in \mathbb{I}^{\jmath}$ (for ${ }_{t i} \mathbf{a}$ as in Section [5.2), or $\lambda=\mu\langle\mathbf{a}\rangle$ if $\mathfrak{g}=\mathfrak{s o}_{2 m+1}$. Recall the bimodules $\mathscr{E}_{i} 1_{\mathbf{a}}$, $\mathscr{F}_{i} 1_{\mathbf{a}}$ from Section $\left[5.2\right.$, and let $\widehat{\mathscr{E}}_{i} 1_{\mathbf{a}}, \widehat{\mathscr{F}}_{i} 1_{\mathbf{a}}$ be their completions with respect to their grading.

Lemma 7.4. We have

$$
\widehat{\mathbb{V}}\left(\mu_{\left\langle_{+i} \mathbf{a}\right\rangle} \Phi(V)_{\mu\langle\mathbf{a}\rangle}\right)=\widehat{\mathscr{E}}_{i} 1_{\mathbf{a}}, \quad \widehat{\mathbb{V}}\left(\mu_{\left\langle_{-i} \mathbf{a}\right\rangle} \Phi(V)_{\mu\langle\mathbf{a}\rangle}\right)=\widehat{\mathscr{F}_{i}} 1_{\mathbf{a}}
$$

Proof. The image $\widehat{\mathbb{V}}\left(\mu_{\left.\alpha_{+} \mathbf{a}\right\rangle} \Phi(V)_{\mu\langle\mathbf{a}\rangle}\right)$ is a completed singular Soergel bimodule. Its rank as a left module over $\widehat{R}^{+i}$ a is the number $\ell$ of weights $\nu$ of $V$ such that $\mu\langle\mathbf{a}\rangle+\nu$ is in the dot-orbit of $\mu\left\langle_{+i} \mathbf{a}\right\rangle$. This is precisely $a_{i}-a_{i-1}$ if $i>\diamond$ and $2 a_{\diamond}$ if $i=\diamond$.

We claim that $\widehat{\mathscr{E}}_{i} 1_{\mathbf{a}}$ is the only completed singular Soergel bimodule with this property. By Wil11, Theorem 1], the indecomposable singular Soergel bimodules are in bijection with the cosets $W_{+i} \backslash W / W_{\mathbf{a}}$. By [Wil11, Section 7.5], the rank of the bimodule associated to a longest double coset representative $w$ as a free left $\widehat{R}^{+i}$ a module is the sum $\sum_{u \in W / W_{\mathbf{a}}} p_{w, u}^{\mathbf{a}}(1)$ where $p_{w, u}^{\mathbf{a}}(x)$ is the parabolic Kazhdan-Lusztig polynomial. The constant term of $p_{w, u}^{\mathbf{a}}(x)$ is 1 , and all its coefficients are non-negative by [D89, 3.11, 4.1], so $p_{w, u}^{\mathbf{a}}(1) \geq 1$ whenever $u W_{\mathbf{a}} \leq w W_{\mathbf{a}}$ in Bruhat order. Thus, the rank of an indecomposable singular Soergel bimodule over $\widehat{R}^{+i}$ a corresponding to $w$ is at least the number of left cosets less than $w W_{\mathbf{a}}$ in Bruhat order. This number is $\ell$ for the double coset of the identity, and thus $>\ell$ for any other coset.

Thus, $\widehat{\mathscr{E}}_{i} 1_{\mathbf{a}}$ is the unique singular Soergel bimodule with rank $\ell$, which shows the equality $\widehat{\mathbb{V}}\left({ }_{\mu\left\langle_{+} \mathbf{a}\right\rangle} \Phi(V)_{\mu\langle\mathbf{a}\rangle}\right) \cong \widehat{\mathscr{E}}_{i} 1_{\mathbf{a}}$. The same argument with left and right hand actions reversed shows that $\left.\widehat{\mathbb{V}}\left(\mu_{-i} \mathbf{a}\right\rangle \mid(V)_{\mu\langle\mathbf{a}\rangle}\right) \cong \widehat{\mathscr{F}_{i}} 1_{\mathbf{a}}$. 
For $\mu=\mu\langle\mathbf{a}\rangle, \lambda=\mu\langle\mathbf{b}\rangle$, recall that by Proposition $5.3(\mathrm{a})$ the category $\mathfrak{F}(\mathbf{a}, \mathbf{b})$ defined in Section 5.2 is a full subcategory in $R^{\mathbf{a}}$-mod- $R^{\mathbf{b}}$, hence we have an obvious completion functor $\widehat{\imath} \mathfrak{F}(\mathbf{a}, \mathbf{b}) \rightarrow \widehat{R}^{\mathbf{a}}$-mod- $\widehat{R}^{\mathbf{b}}$.

Lemma 7.5. There exists a unique functor $\mathbb{U}: \mathfrak{F}(\mathbf{a}, \mathbf{b}) \rightarrow{ }_{\lambda} \widehat{\mathcal{P}}_{\mu}$ such that $\widehat{\mathbb{V}} \circ \mathbb{U}: \mathfrak{F}(\mathbf{a}, \mathbf{b}) \rightarrow$ $\widehat{R}^{\mathbf{a}}$-mod- $\widehat{R}^{\mathbf{b}}$ is the completion functor.

Proof. By Corollaryl7.2, the functor $\widehat{\mathbb{V}}$ induces an equivalence between ${ }_{\lambda} \widehat{\mathcal{P}}_{\mu}$ and its image. Hence to prove the lemma, it is enough to prove that the image of $\mathfrak{F}(\mathbf{a}, \mathbf{b})$ under the completion functor lands in $\mathbb{V}\left({ }_{\lambda} \widehat{\mathcal{P}}_{\mu}\right)$. This is a consequence of (7.1) and Lemma 7.3,

This shows that the 2-category $\mathfrak{F}_{r, m}$ has 2-representations given by sending a to the category $\mu\langle\mathbf{a}\rangle \mathcal{M}$, or more generally, to any subcategory of ${ }_{\mu}\langle\mathbf{a}\rangle \mathcal{M}$ closed under the action of projective functors. Composing with $\Gamma: \mathfrak{U}_{r}^{\jmath} \rightarrow \mathfrak{F}_{r, m}$ from (5.6), we obtain the following theorem.

Theorem 7.6. The category $\mathfrak{U}_{r}^{\jmath}$ has a representation sending $\lambda$ in $\mathrm{X}_{\jmath}$ to the category ${ }_{\mu}\langle\mathbf{a}\rangle \mathcal{M}$ if $\lambda=\lambda(\mathbf{a})$ for $\mathbf{a} \in \Sigma_{r, m}$, and to zero otherwise. This action descends to the intersection of $\mu\langle\mathbf{a}\rangle \mathcal{M}$ with any subcategory of $U-\bmod$ which is closed under the action of projective functors, including:

- the subcategory of finite length U-modules,

- the subcategory of finite dimensional U-modules,

- the subcategory of modules locally finite for a subalgebra $\mathfrak{k} \subset \mathfrak{g}$,

- the BGG category $\mathcal{O}$ and its parabolic generalizations $\mathcal{O}^{\mathfrak{p}}$,

- the subcategory of projective-injective modules in these categories.

Tracing through the definitions, this action sends the 1-morphisms $\mathcal{E}_{ \pm i}$ to the translation functor from the block $\mu_{\mu \mathbf{a}\rangle} \mathcal{M}$ to ${ }_{ \pm i} \mu\langle\mathbf{a}\rangle \mathcal{M}$. By (7.1), these are always given by summands of the functor $V \otimes-$ of tensor product with the defining representation. In future work, we will expand the discussion of this categorical action in greater detail and describe further applications.

\section{Appendix A. Categorification of the -Serre Relations}

In this appendix we shall derive some bubble slide formulas. We then prove the identities (4.11) and (4.12). This completes the proof of Proposition 4.3 on the categorification of the j-Serre relations.

A.1. Bubble slides. We first provide several bubble slide formulas for $\mathfrak{U}^{\mathfrak{\jmath}}$, which are the counterparts of Lauda's bubble slide formulas [La10, Propositions 5.6, 5.7]. We recall our convention that all the strands without labels should be viewed as labeled by $\diamond$.

Lemma A.1 (Bubble slides). The following relations hold for all $\lambda \in \mathrm{X}_{\jmath}\left(\right.$ recall $\left.\lambda_{\diamond}=\left\langle{ }^{\theta} \alpha_{\diamond}^{\vee}, \lambda\right\rangle\right)$ :

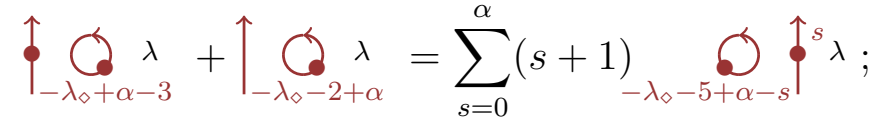

$$
\begin{aligned}
& \bigodot_{\lambda_{\diamond}+\alpha+1} \uparrow \lambda+\underset{\lambda_{\diamond}+2+\alpha}{\oint^{\prime}} \uparrow \lambda=\sum_{s=0}^{\alpha}(s+1) \uparrow_{\lambda_{\diamond}-1+\alpha-s}^{s} ;
\end{aligned}
$$




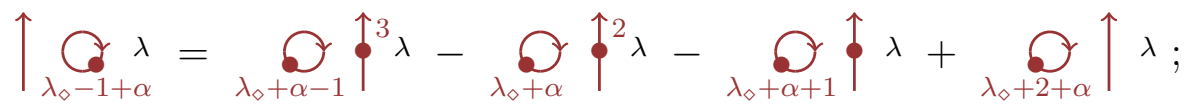

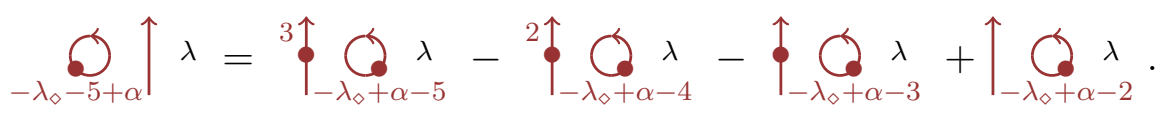

Proof. We shall first prove the case when $\lambda_{\diamond} \geq-2$. Note that in this case only real bubbles appear on the left hand side of the equation (A.2), since bubbles with negative degree are set to be 0 . We shall proceed in the following steps:

(a) Prove (A.1) for $\alpha \geq \lambda_{\diamond}+3$, and prove (A.2) for all $\alpha$;

(b) Prove (A.3) by using (A.2);

(c) To complete the proof of (A.1) for $0 \leq \alpha \leq \lambda_{\diamond}+2$ (fake bubbles), we use induction on $\alpha$ based on the definition of fake bubbles, with the help of (A.3.

(d) Prove (A.4) by using (A.1) (similar to (b)).

We now proceed with Step (a).

From the relation (3.14), we have (for $m \geq 0$ )

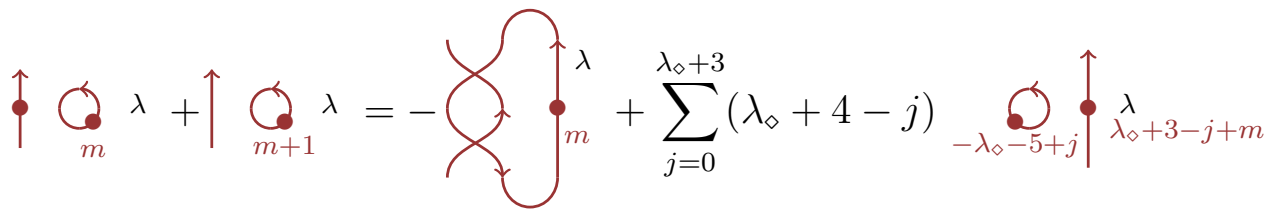

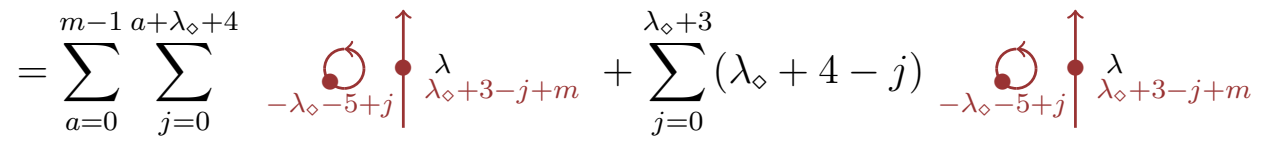

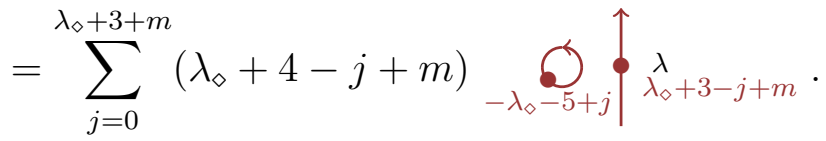

Replacing $m+\lambda_{\diamond}+3=\alpha$ above, we have proved (A.1) for $\alpha \geq \lambda_{\diamond}+3$ (thanks to $m \geq 0$ ).

In an entirely similar way, we can prove (for $\alpha \geq-1-\lambda_{\diamond}$ ) :

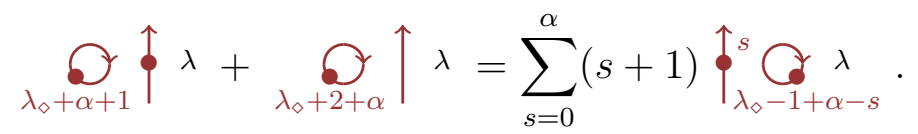

On the other hand, if $\alpha \leq-2-\lambda_{\diamond}$, then $\alpha \leq 0$ by the assumption in (a). If $\alpha<0$, both left and right hand sides of the identity (A.2) are 0. If $\alpha=0$ (which is non-trivial only when $\lambda_{\diamond}=-2$ ), then both sides are equal to $2 \uparrow \lambda$. So we have established (A.2) for all $\alpha$.

Let us proceed with Step (b). We can rewrite the right hand side of (A.3) as follows:

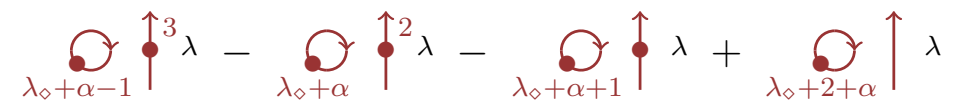

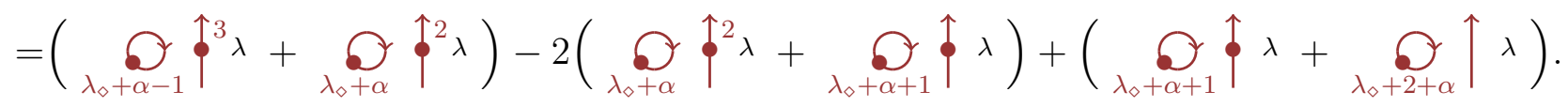

Then by applying (A.2) three times, we obtain the identity (A.3). 
Now we proceed with Step (c), that is, we prove (A.1) for $0 \leq \alpha \leq \lambda_{\diamond}+2$ by induction on $\alpha$. Thanks to the bubble relations (3.7)-(3.9), we have

$$
\bigotimes_{-\lambda_{\diamond}-2}^{\lambda}=1, \quad \bigodot_{\lambda_{\diamond}-1}^{\lambda}=2
$$

and a recursive definition of fake bubbles as follows, for $\alpha>0$ :

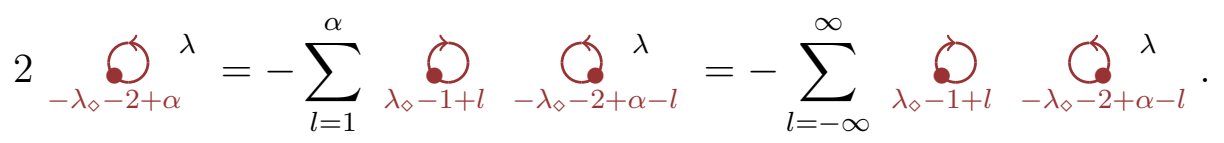

The base case $\alpha=0$ of $(\underline{A .1})$ is trivial, since both sides of identity (A.1) are $\uparrow^{\lambda}$. The case $\alpha=1$ of (A.1) can be verified using (A.3) by writing the fake bubbles in terms of the real ones using (A.5). For $\alpha>1$, it follows by (A.5), the inductive assumption, and (A.3) that

$$
\begin{aligned}
& 2\left(\uparrow_{-\lambda_{\diamond}+\alpha-3}^{\widehat{Q}} \lambda+\uparrow_{-\lambda_{\diamond}-2+\alpha}^{\underset{Q_{2}}{\lambda}}\right)
\end{aligned}
$$

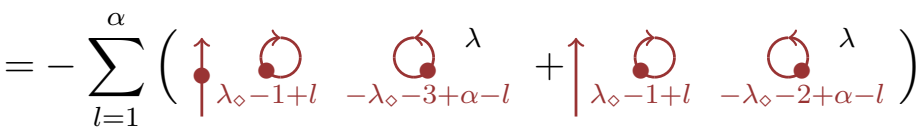

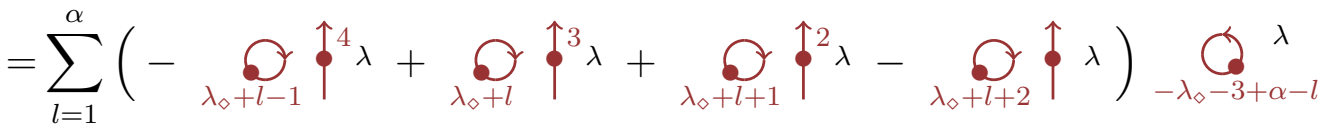

$$
\begin{aligned}
& +\sum_{l=1}^{\alpha}\left(-\bigodot_{\lambda_{\diamond}+l-1}^{\uparrow^{3} \lambda}+\bigodot_{\lambda_{\diamond}+l}^{\diamond} \uparrow^{2} \lambda+\bigodot_{\lambda_{\diamond}+l+1}^{\uparrow^{\prime}} \lambda-\bigodot_{\lambda_{\diamond}+l+2}^{\diamond} \uparrow \lambda\right) \underset{-\lambda_{\diamond}-2+\alpha-l}{\bigotimes^{\lambda}} .
\end{aligned}
$$

To simplify the right-hand side above, we will compute 4 sums (each of 2 summands which line up vertically) as follows. First, by the induction hypothesis and using the relation (3.9), we have

$$
\begin{aligned}
& \sum_{l=1}^{\alpha}\left(-\underset{\lambda_{\diamond}+l-1}{\uparrow^{4}} \underset{-\lambda_{\diamond}-3+\alpha-l}{\bigotimes^{\lambda}}\right)+\sum_{l=1}^{\alpha}\left(-\underset{\lambda_{\diamond}+l-1}{\bigcirc^{3}} \underset{-\lambda_{\diamond}-2+\alpha-l}{\bigotimes^{\lambda}}\right) \\
& =\sum_{l=1}^{\alpha}\left(-\bigcup_{\lambda_{\diamond}-1+l}\left(\sum_{s=0}^{\alpha-l}(s+1) \underset{-\lambda_{\diamond}-5+\alpha-l \perp_{s}}{\overbrace{s}^{\lambda+3}}\right)\right)
\end{aligned}
$$

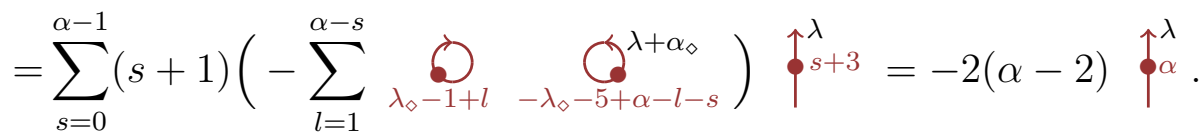

Similarly we have

$$
\begin{aligned}
& \sum_{l=1}^{\alpha}\left(\underset{\lambda_{\diamond}+l}{\diamond \uparrow^{3}} \underset{-\lambda_{\diamond}-3+\alpha-l}{\bigotimes^{\lambda}}\right)+\sum_{l=1}^{\alpha}\left(\underset{\lambda_{\diamond}+l}{\diamond \uparrow^{2}} \underset{-\lambda_{\diamond}-2+\alpha-l}{\bigotimes_{\alpha}^{\lambda}}\right)=2(\alpha-1) \oint^{\alpha},
\end{aligned}
$$

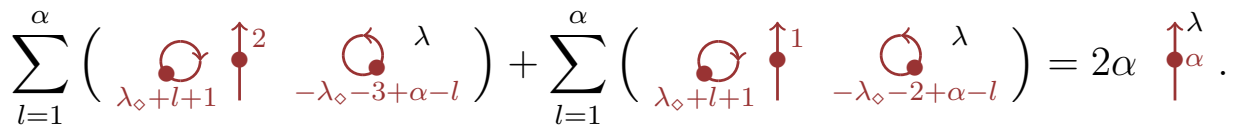


Finally, by the definition of fake bubbles in (A.5) we have

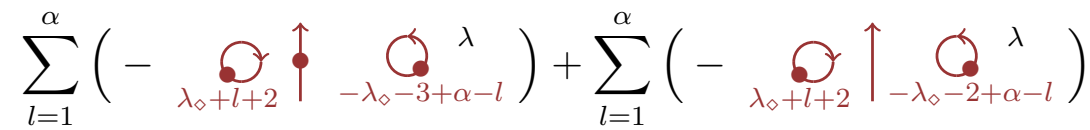

$$
\begin{aligned}
& =\sum_{s=0}^{\alpha-1}(s+1)\left(-\sum_{l=1}^{\alpha-s} \underset{\lambda_{\diamond}+2+l}{\bigcup_{-\lambda_{\diamond}-5+\alpha-l-s}} \underset{\oint^{\lambda+\alpha_{\diamond}}}{\oint^{\lambda}} s\right. \\
& =2 \sum_{s=0}^{\alpha-1}(s+1) \underset{-\lambda_{\diamond}-5+\alpha-s}{0} \uparrow_{s}^{s} .
\end{aligned}
$$

Summing up the above 4 identities, we finish the proof of (A.1).

The proof of Step (d) that the identity (A.4) follows from (A.1) is entirely similar to the proof of Step (b) that the (A.3) follows by (A.2). We skip the detail.

This finishes the proof of the bubble slide formulas for the case $\lambda_{\diamond} \geq-2$. The case $\lambda_{\diamond} \leq-3$ is entirely similar, where the left hand side of the identity (A.1) involves only real bubble, hence can be easily proved, as well as the identity (A.4). The proof of the identities (A.2) and (A.3) follows a similar argument as above. Alternatively, we could simply apply symmetries of $\mathfrak{U}^{\mathfrak{j}}$ in Section 3.3.

Corollary A.2. The following relations hold for all $\lambda \in \mathrm{X}_{\jmath}$ :

$$
\begin{aligned}
& \uparrow_{-\lambda_{\diamond}-2+\alpha}^{\bigotimes_{\lambda}}=\sum_{s=0}^{\alpha}\left\lceil\frac{s+1}{2}\right\rceil \underset{-\lambda_{\diamond}-5+\alpha-s}{\bigotimes_{i}^{s} \lambda} ; \\
& \underset{\lambda_{\diamond}+2+\alpha}{\diamond}\left\lceil\lambda=\sum_{s=0}^{\alpha}\left\lceil\frac{s+1}{2}\right\rceil \uparrow_{\lambda_{\diamond}-1+\alpha-s}^{s} \bigotimes_{\lambda}^{\lambda} .\right.
\end{aligned}
$$

Proof. The proof follows from Lemma A.1 by induction on $\alpha$.

We can also formulate the downward arrow counterparts of the bubble slide relations in Lemma A.1. The proof is similar, hence shall be omitted.

Proposition A.3. The following relations hold for all $\lambda \in \mathrm{X}_{\jmath}$ :

$$
\begin{aligned}
& \downarrow \underset{\lambda_{\diamond}+\alpha-2}{\mathrm{Q}^{\lambda}}+\downarrow \underset{\lambda_{\diamond}-1+\alpha}{\mathrm{Q} \lambda}=\sum_{s=0}^{\alpha}(s+1) \underset{\lambda_{\diamond}-4+\alpha-s}{\bigcirc^{\prime}} \downarrow^{s} \lambda \text {; }
\end{aligned}
$$

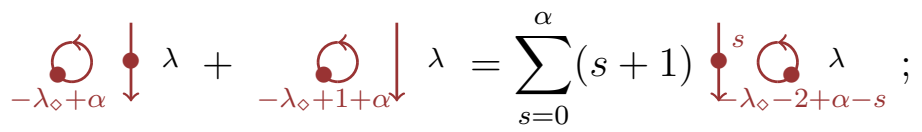

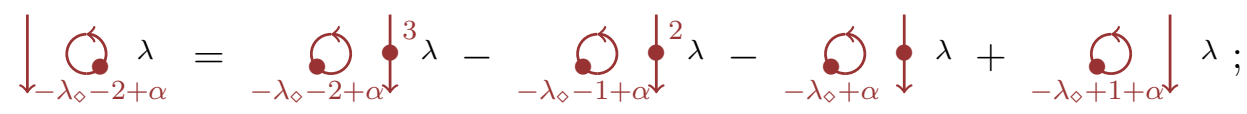

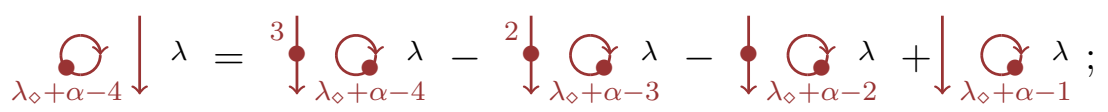




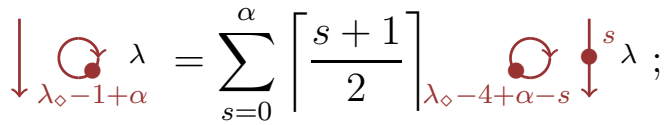

$$
\begin{aligned}
& \bigotimes_{-\lambda_{\diamond}+1+\alpha \downarrow} \lambda=\sum_{s=0}^{\alpha}\left\lceil\frac{s+1}{2}\right\rceil \downarrow_{-\lambda_{\diamond}-2+\alpha-s}^{s} \bigcup_{\lambda} .
\end{aligned}
$$

A.2. Proof of Proposition A.6. In this subsection, we shall first establish a variant of the identity (4.11), as a preparation toward the proof of the identity (4.11).

Let $\lambda \in \mathrm{X}_{\jmath}$ with $\lambda_{\diamond} \geq 2$. Recall the 2-morphism $\kappa \in \operatorname{End}\left(\mathcal{E}_{\diamond} \mathcal{F}_{\diamond}^{2} 1_{\lambda}\right)$ in Lemma 4.1. The following lemma follows from the proof therein.

Lemma A.4. We have $\kappa \cdot \iota_{t}=0$, for $0 \leq t \leq \lambda_{\diamond}-3$.

Let us define

$$
\kappa_{2}=\frac{1}{2} \sum_{a+b+c=-2} \text { and } \kappa_{3}=\frac{1}{2} \sum_{a+b+c=-3}^{\lambda}
$$

Recall the definition of $\kappa$ in Lemma 4.1 uses $\iota_{s}$, which consists of the top parts of $\kappa_{2}$ and $\kappa_{3}$. An alternative way of decomposing $\kappa$ is available by use of the low parts of $\kappa_{2}$ and $\kappa_{3}$. To that end, we set

$$
\tilde{\pi}_{s}=\overbrace{}^{s} \text { and } \tilde{\iota}_{s}=-\frac{1}{2} \sum_{a+c=-s-2} \underbrace{}_{a}+\frac{1}{2} \sum_{a+c=-s-3}\}_{a}
$$

Then clearly we also have

$$
\kappa=\mathrm{id}_{\mathcal{E}_{\diamond} \mathcal{F}_{\diamond}^{(2)} 1_{\lambda}}-\sum_{0 \leq s \leq \lambda_{\diamond}-3} \tilde{\iota}_{s} \tilde{\pi}_{s}
$$

The following lemma is a counterpart of Lemma A.4, whose proof is skipped.

Lemma A.5. We have $\tilde{\pi}_{t} \tilde{\iota}_{s}=\delta_{s, t} \operatorname{id}_{\mathcal{F}_{\diamond} 1_{\lambda}}$, for $0 \leq s \leq \lambda_{\diamond}-3$ and $0 \leq t \leq \lambda_{\diamond}-3$. Moreover, we have $\tilde{\pi}_{t} \cdot \kappa=0$ for $0 \leq t \leq \lambda_{\diamond}-3$.

Recall the diagrams $B_{1}, B_{2}, P_{k}, C_{1}, C_{2}$ and $I_{k}$, for $0 \leq k \leq \lambda_{\diamond}-1$, from (4.7)-(4.8). We shall establish first a simpler version of the identity (4.11).

Proposition A.6. The following identity holds in $\tilde{\mathfrak{U}}^{\jmath}$ :

$$
\left[\begin{array}{c}
B_{1} \\
\kappa \cdot B_{2} \\
P_{0} \\
\vdots \\
P_{\lambda_{\diamond}-1}
\end{array}\right] \cdot\left[\begin{array}{lllll}
C_{1} & C_{2} \cdot \kappa & I_{0} & \cdots & I_{\lambda_{\diamond}-1}
\end{array}\right]=i d_{\left(\lambda_{\diamond}+2\right) \times\left(\lambda_{\diamond}+2\right)} .
$$


The proof of the identity in Proposition A.6 is divided into Lemmas A.7 A.13 below. As we shall not need the relation (3.17) in the process, the identity holds in $\tilde{\mathfrak{U}}^{3}$.

Lemma A.7. We have $B_{2} \cdot C_{1}=0$ and $B_{1} \cdot C_{2}=0$.

Proof. We have

$$
B_{1} \cdot C_{2}=-\underbrace{\lambda}=-\underbrace{\lambda}=0 .
$$

The proof for the second identity is similar and will be skipped.

Lemma A.8. We have $P_{k} \cdot I_{\ell}=\delta_{k, \ell} \operatorname{id}_{\mathcal{F}_{\diamond} 1_{\lambda}}$, for $0 \leq k, \ell \leq \lambda_{\diamond}-1$.

Proof. By the bubble relation (3.9), we compute

$$
\left.P_{k} \cdot I_{\ell}=\frac{1}{2} \sum_{s+t=l} \downarrow_{-\lambda_{\diamond}-2+t}^{\bigotimes_{-1-k+s}}=\delta_{k, l}\right\rfloor .
$$

Lemma A.9. We have $B_{1} \cdot I_{k}=0$ and $P_{k} \cdot C_{1}=0$, for $0 \leq k \leq \lambda_{\diamond}-1$.

Proof. We have

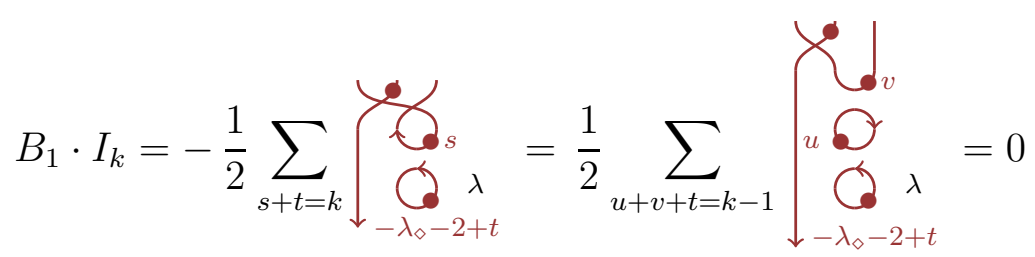

thanks to the vanishing of bubbles of negative degree in relations (3.7)-(3.8).

Again because of the vanishing of bubbles of negative degree in relation (3.7), we have

$$
P_{k} \cdot C_{1}=\overbrace{\lambda}^{\lambda_{\diamond}-1-k}=0 .
$$

Lemma A.10. We have $\kappa \cdot B_{2} \cdot I_{k}=0$, for $0 \leq k \leq \lambda_{\diamond}-1$.

Proof. We have

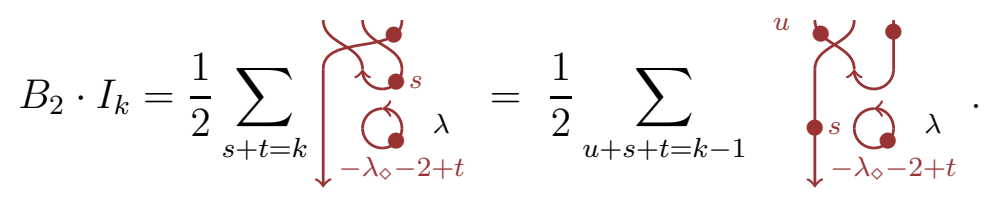

Hence $B_{2} \cdot I_{k}$ is of the form

$$
B_{2} \cdot I_{k}=\sum_{0 \leq t \leq \lambda_{\diamond}-3} \iota_{t} \cdot x_{t}
$$


for some suitable $x_{t}$. By Lemma A.4, we have

$$
\kappa \cdot B_{2} \cdot I_{k}=\sum_{t} \kappa \iota_{t} x_{t}=0 .
$$

Lemma A.11. We have $P_{k} \cdot C_{2} \cdot \kappa=0$, for $0 \leq k \leq \lambda_{\diamond}-1$.

Proof. We have

which is of the form

$$
P_{k} \cdot C_{2}=\overbrace{}^{\lambda}{ }^{\lambda},
$$

$$
P_{k} \cdot C_{2}=\sum_{0 \leq s \leq \lambda_{\diamond}-2} y_{s} \tilde{\pi}_{s}
$$

for some suitable $y_{s}$. This implies by Lemma A.5 that $P_{k} \cdot C_{2} \cdot \kappa=0$.

Lemma A.12. We have $B_{1} \cdot C_{1}=\mathrm{id}_{\mathcal{F}_{\diamond}^{(2)} \mathcal{E}_{\diamond} 1_{\lambda}}$.

Proof. Using equation (3.15), we compute that

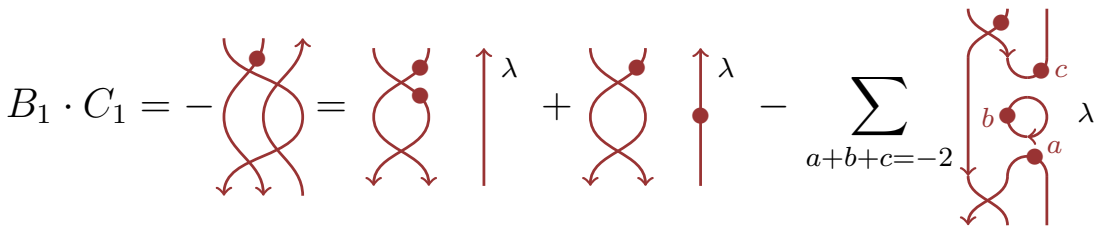

$$
\begin{aligned}
& =\searrow^{\uparrow}{ }^{\lambda},
\end{aligned}
$$

where

$$
\sum_{a+b+c=-2} \overbrace{b} \lambda=0, \quad \text { for } \lambda_{\diamond} \geq 2
$$

because of the bubble relation (3.7).

Lemma A.13. We have $\kappa \cdot B_{2} \cdot C_{2}=\kappa$.

Proof. We compute that

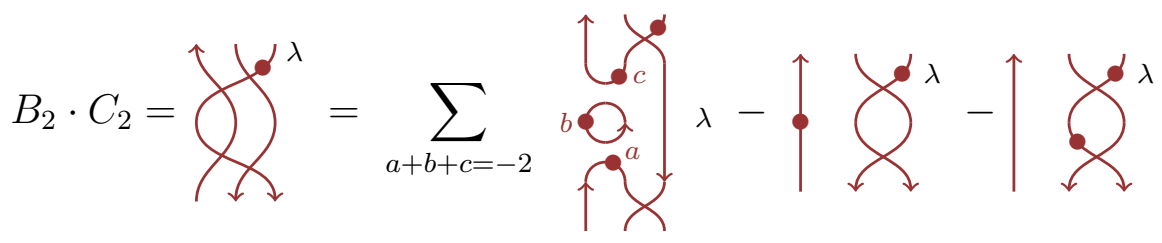

Note that the third summand above equals $\mathrm{id}_{\mathcal{E}_{\diamond} \mathcal{F}_{\diamond}^{(2)} 1_{\lambda}}$ and the second equals 0 . Denote by $Y$ the first summand above. It remains to show that $\kappa \cdot Y=0$. Note that $Y$ is of the form $Y=\sum_{0 \leq t \leq \lambda_{\diamond}-3} \iota_{t} z_{t}$ for some $z_{t}$. This implies by Lemma A.4 that $\kappa \cdot Y=0$. The lemma follows. 
Therefore, we have completed the proof of Proposition A.6.

A.3. Proof of the identity (4.11). Now we would like to modify some 2-morphisms involved in Proposition A.6. Introduce

$$
\begin{aligned}
& A=\bigcup_{\lambda}, \quad B=\frac{1}{2} \sum_{u+s+t=-3} u_{Q^{t}} \bigcup_{s}, \quad C=\psi / \lambda,
\end{aligned}
$$

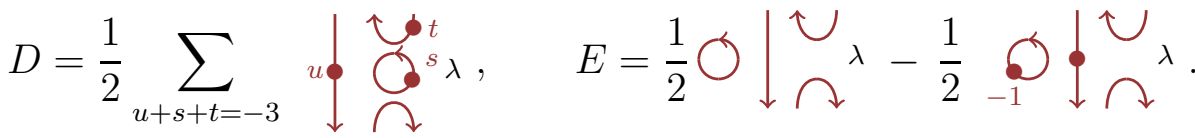

We define $I_{\lambda_{\diamond}-1}^{\prime}=(1-C+D) \cdot I_{\lambda_{\diamond}-1}$. It is easy to show that

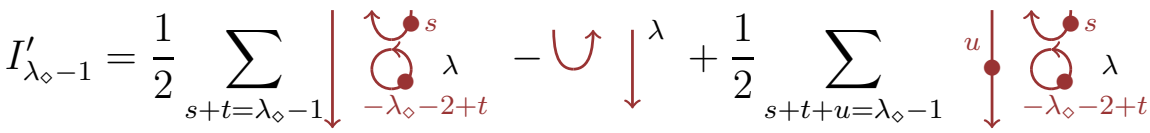

$$
\begin{aligned}
& =I_{\lambda_{\diamond}-1}-I(2)+I(3) \text {. }
\end{aligned}
$$

The second line above defines $I(2)$ and $I(3)$ as the second and third summands without signs in the first line; these notations will be used below.

We also define $P_{0}^{\prime}=P_{0} \cdot(1-A+B-E)$. Then we can show readily that

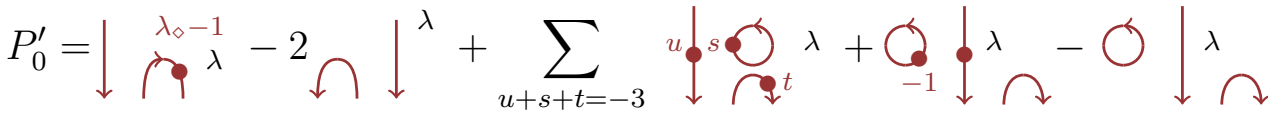

$$
\begin{aligned}
& =P_{0}-P(2)+P(3)-P(4)+P(5) \text {. }
\end{aligned}
$$

The second line above defines $P(2), P(3), P(4)$, and $P(5)$ as the second to fifth summands without signs in the first line.

We would like to redo Proposition A.6 with $I_{\lambda_{\diamond}-1}$ and $P_{0}$ replaced by $I_{\lambda_{\diamond}-1}^{\prime}$ and $P_{0}^{\prime}$, respectively.

Proposition A.14. The identity (4.11) holds, that is, we have

$$
\left[\begin{array}{c}
B_{1} \\
\kappa \cdot B_{2} \\
P_{0}^{\prime} \\
\vdots \\
P_{\lambda_{\diamond}-1}^{\prime}
\end{array}\right] \cdot\left[\begin{array}{lllll}
C_{1} & C_{2} \cdot \kappa & I_{0} & \cdots & I_{\lambda_{\diamond}-1}^{\prime}
\end{array}\right]=i d_{\left(\lambda_{\diamond}+2\right) \times\left(\lambda_{\diamond}+2\right)} .
$$

Thanks to Proposition A.6, we only need to consider the relations involving $P_{0}^{\prime}$ or $I_{\lambda_{\diamond}-1}^{\prime}$. The computation is divided into Lemmas A.15 A.21 below.

Lemma A.15. We have $B_{1} \cdot I_{\lambda_{\diamond}-1}^{\prime}=0$. 
Proof. Recall from (A.8) that $I_{\lambda_{\diamond}-1}^{\prime}=I_{\lambda_{\diamond}-1}-I(2)+I(3)$. By Lemma A.9 that $B_{1} \cdot I_{\lambda_{\diamond}-1}=0$. By the same argument as for Lemma A.9, we show that $B_{1} \cdot I(3)=0$. Finally, we have

$$
B_{1} \cdot I(2)=\overbrace{\lambda}=0 .
$$

The lemma is proved.

Lemma A.16. We have $\kappa \cdot B_{2} \cdot I_{\lambda_{\diamond}-1}^{\prime}=0$.

Proof. We compute that

$$
B_{2} \cdot I(3)=\sum_{a+b+t=\lambda_{\diamond}-1} \underbrace{a}_{\downarrow-\lambda_{\diamond}-2+t}=\sum_{t+s=-1}
$$

Observe that $B_{2} \cdot I(k)$ for $k=2,3$ are of the form

$$
B_{2} \cdot I(k)=\sum \iota_{t} \cdot \alpha_{t}
$$

for some $\alpha_{t}$, and this implies by Lemma A.4 that $\kappa \cdot B_{2} \cdot I(k)=0$. Recall $\kappa \cdot B_{2} \cdot I_{\lambda_{\diamond}-1}=0$ from Lemma A.10, The lemma follows.

Lemma A.17. We have $P_{k} \cdot I_{\lambda_{\diamond}-1}^{\prime}=0$, for $0<k<\lambda_{\diamond}-1$.

Proof. We have $P_{k} \cdot I_{\lambda_{\diamond}-1}=0$ by Lemma A.8. Now we compute that

$$
\begin{aligned}
P_{I} \cdot(-I(2)+I(3)) & =-\left.\int^{\lambda}\right|^{\lambda}+\frac{1}{2} \sum_{u+s+t=\lambda_{\diamond}-1}{\underset{s}{s+\lambda_{\diamond}-1-k}}_{\downarrow_{\lambda_{\diamond}-1-k}^{u}}^{\lambda \lambda_{\diamond}-2-t} \\
& =-\downarrow_{\downarrow_{\diamond}-1-k}^{\lambda}=0 .
\end{aligned}
$$

The lemma follows.

Lemma A.18. We have $P_{0}^{\prime} \cdot C_{2} \cdot \kappa=0$.

Proof. Recall from (A.9) that $P_{0}^{\prime}=P_{0}-P(2)+P(3)-P(4)+P(5)$. First we note that

$$
P(4) \cdot C_{2}=0 \gamma^{\lambda}=0 \text {; }
$$

similarly it can be shown that $P(5) \cdot C_{2}=0$.

On the other hand, we compute that

$$
\left.P(2) \cdot C_{2}=\mathcal{Y}^{\lambda}=\sum_{a+b=-1}\right\}^{\lambda},
$$


and

$$
P(3) \cdot C_{2}=\sum_{u+s+t=-3} \sum_{u+s+t=-4}(u+1)^{\lambda}=\sum^{\lambda} .
$$

The particular forms of $P(k) \cdot C_{2}$, for $k=2,3$, allow us to apply Lemma A.5 to conclude that $P(k) \cdot C_{2} \cdot \kappa=0$. Note $P_{0} \cdot C_{2} \cdot \kappa=0$ by Lemma A.11. The lemma now follows.

Lemma A.19. We have $P_{0}^{\prime} \cdot C_{1}=0$.

Proof. First we compute that

$$
P(2) \cdot C_{1}=\varkappa^{\lambda}=0 .
$$

On the other hand, recall from Lemma A.9 that $P_{0} \cdot C_{1}=0$ thanks to the vanishing of bubbles of negative degrees. Similar computations show that $P(i) \cdot C_{1}=0(i=3,4,5)$ again thanks to the relations (3.7) and (3.8). The lemma is proved.

Lemma A.20. We have $P_{0}^{\prime} \cdot I_{k}=0$, for $0<k<\lambda_{\diamond}-1$.

Proof. Recall $P_{0} \cdot I_{k}=0$ by Lemma A.8, We now compute that

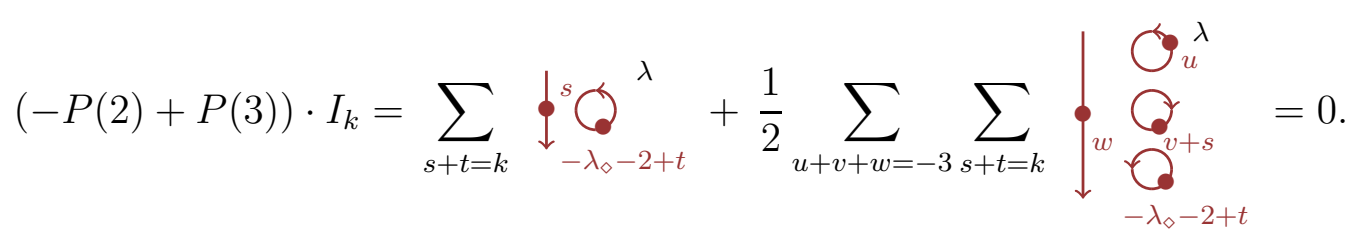

by the bubble relation (3.9). In addition, thanks to the vanishing of bubbles of negative degrees, we have $P(i) \cdot I_{k}=0$ for $i=4,5$. The lemma follows.

Lemma A.21. We have $P_{0}^{\prime} \cdot I_{\lambda_{\diamond}-1}^{\prime}=0$.

Proof. We compute that

$$
\begin{aligned}
& P_{0}^{\prime} \cdot I_{\lambda_{\diamond}-1}^{\prime}=P_{0} \cdot I_{\lambda_{\diamond}-1}-\downarrow_{\downarrow_{\diamond}-1}^{\lambda}+\frac{1}{2} \sum_{u+s+t=\lambda_{\diamond}-4} \downarrow_{\downarrow}^{u} \Theta_{t}^{\theta_{s}^{\lambda}}-\sum_{s+t=-3} \downarrow^{s} Q_{t}^{\lambda} \\
& +2 \circlearrowleft \downarrow^{\lambda}-\sum_{s+t=-3}(s+1) \downarrow^{s} Q_{t}^{\lambda}+\sum_{s+t=-3} \downarrow{ }^{s} Q_{t}^{\lambda}-\sum_{s+t=-3}(s+1) \downarrow^{s} Q_{t}^{\lambda} \\
& +\frac{1}{2} \sum_{\substack{a+b+c=-3 \\
u+s+t=-3}} \downarrow \bigodot_{t}^{Q^{c+s}}-\circlearrowleft \downarrow^{\lambda}+O \downarrow^{\lambda}-O \downarrow^{\lambda} \\
& +\widehat{Q}_{-1} \downarrow^{\lambda}-\widehat{Q}_{-1} \downarrow^{\lambda}+\mathrm{Q}_{-1} \downarrow^{\lambda} \\
& =\downarrow^{\lambda}+Q_{-1} \downarrow^{\lambda}-\sum_{s+t=-3}(s+1) \downarrow^{s} Q_{t}^{\lambda}=0
\end{aligned}
$$


where the last identity follows from Lemma A.1.

This completes the proof of Proposition A.14.

\section{A.4. Proof of the identity (4.12).}

Proposition A.22. The identity (4.12) holds in $\dot{\mathfrak{U}}^{3}$, that is, we have

$$
=-\left[\begin{array}{lllll}
C_{1} & C_{2} \cdot \kappa & I_{0} & \cdots & I_{\lambda_{\diamond}-1}^{\prime}
\end{array}\right] \cdot\left[\begin{array}{c}
B_{1} \\
\kappa \cdot B_{2} \\
P_{0}^{\prime} \\
\vdots \\
P_{\lambda_{\diamond}-1}
\end{array}\right]
$$

The last identity follows from the relation (3.17). So it suffices to prove only the first identity. We first compute $\left[\begin{array}{ll}C_{1} & C_{2} \cdot \kappa\end{array}\right] \cdot\left[\begin{array}{c}B_{1} \\ \kappa \cdot B_{2}\end{array}\right]$. We list the relevant computation in the following lemma.

Lemma A.23. We have

(a)

$$
C_{1} \cdot B_{1}=-\underbrace{\lambda}=-
$$

(b)

$$
C_{2} \cdot \operatorname{id}_{\mathcal{E}_{\diamond} \mathcal{F}_{\diamond}^{(2)} 1_{\lambda}} \cdot B_{2}=\chi^{\lambda}=X_{\lambda}^{\lambda}
$$

(c)

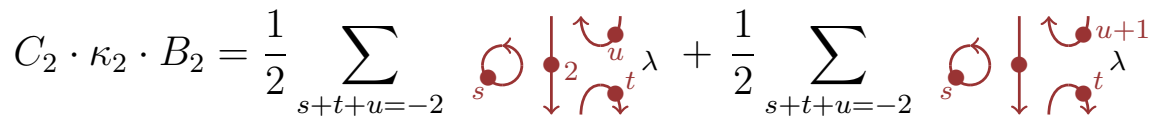

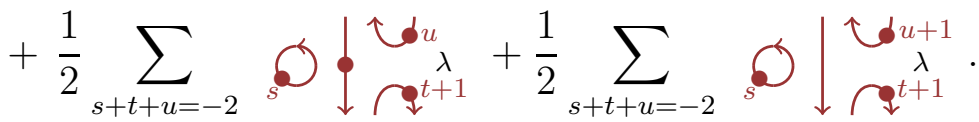


(d)

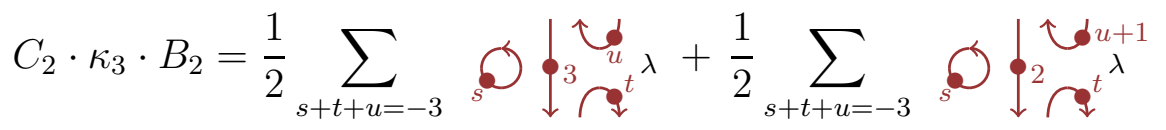

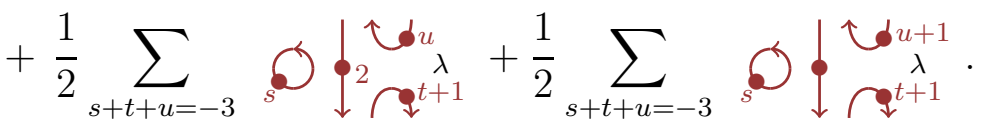

(e)

$$
\begin{aligned}
& C_{2} \cdot \kappa_{2} \cdot B_{2}-C_{2} \cdot \kappa_{3} \cdot B_{2}
\end{aligned}
$$

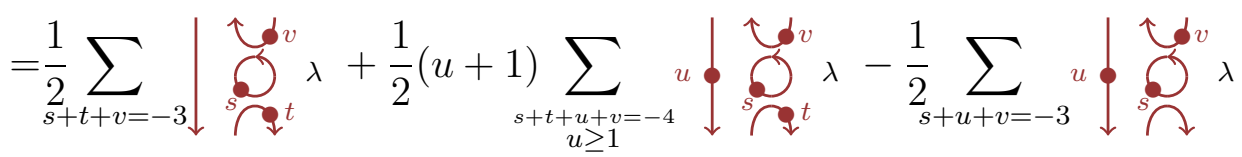

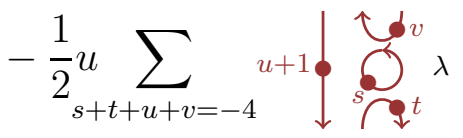

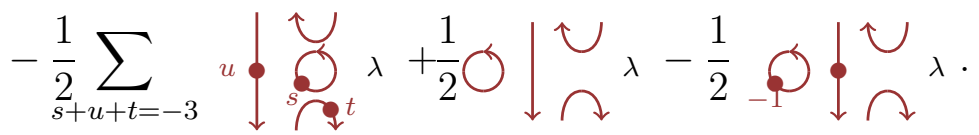

Proof. The first four items are straightforward. Here we show the computation for (e). We remind the reader that we shall use the bubble slides lemma (Lemma A.1) extensively here.

We have

$$
\begin{aligned}
& C_{2} \cdot \kappa_{2} \cdot B_{2}-C_{2} \cdot \kappa_{3} \cdot B_{2}
\end{aligned}
$$

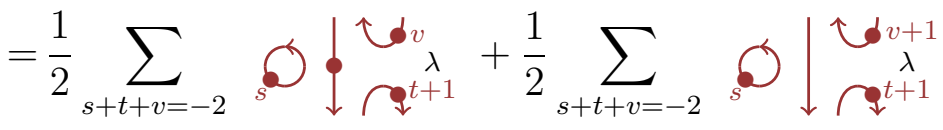

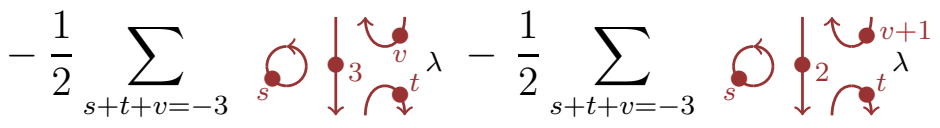

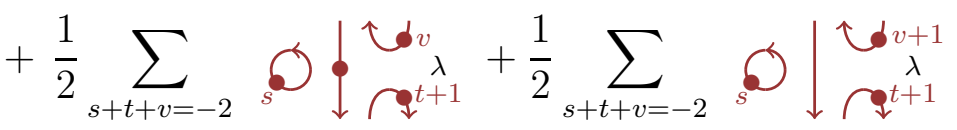

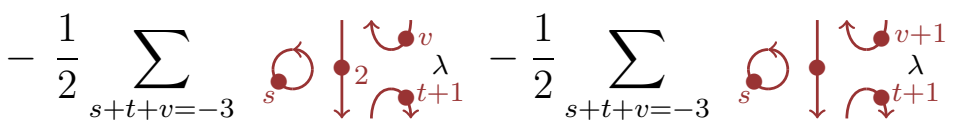

$$
\begin{aligned}
& =\frac{1}{2} \sum_{s+u+v+t+1=-3}(u+1) \downarrow_{\downarrow}^{\mho_{s}^{d} \lambda} \lambda-\left.\frac{1}{2} \sum_{s+t+1=0} \sigma\right|_{s} \lambda
\end{aligned}
$$

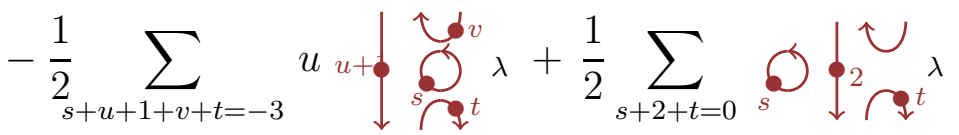

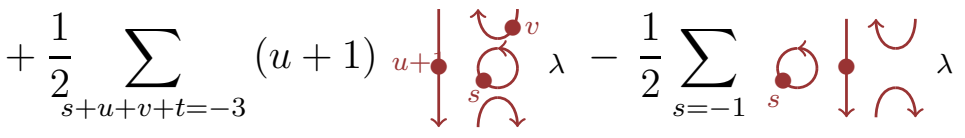




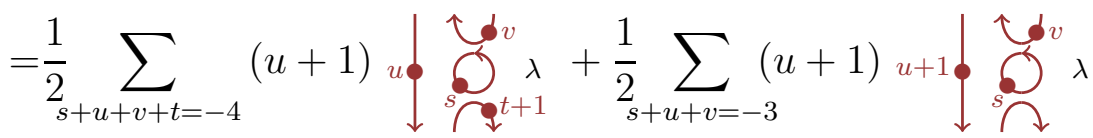

$$
\begin{aligned}
& -\frac{1}{2} \sum_{s+u+v+t=-4} u u+1 \overbrace{s}^{t} \lambda
\end{aligned}
$$

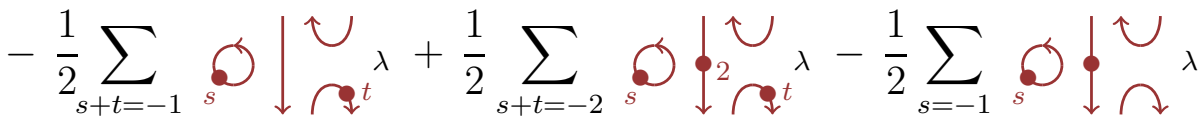

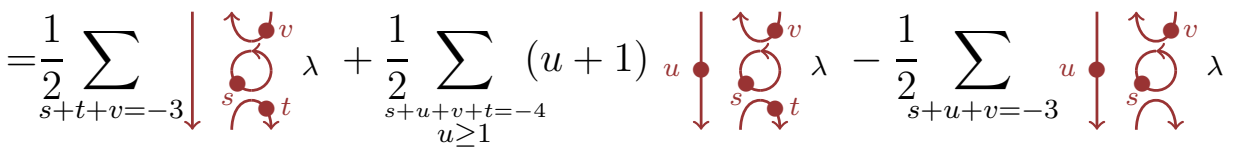

$$
\begin{aligned}
& -\frac{1}{2} \sum_{s+u+v+t=-4} u+1 \underbrace{0}_{s} \lambda
\end{aligned}
$$

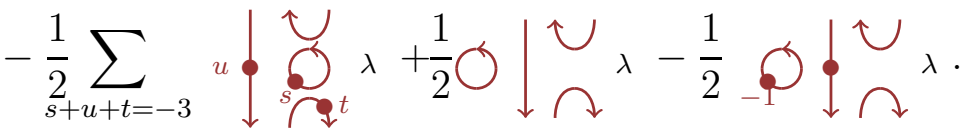

This finishes the computation.

Then we compute the product $\left[\begin{array}{lll}I_{0} & \cdots & I_{\lambda_{\diamond}-1}^{\prime}\end{array}\right] \cdot\left[\begin{array}{c}P_{0}^{\prime} \\ \vdots \\ P_{\lambda_{\diamond}-1}\end{array}\right]$. Recall the definition of $P_{0}^{\prime}$ and $I_{\lambda_{\diamond}-1}^{\prime}$ in (A.8) and (A.9). We list the relevant computation in the following lemma. The computations are straightforward, hence the proof shall be omitted.

Lemma A.24. We have

(a)

(b)

$$
\sum_{k=0}^{\lambda_{\diamond}-1} I_{k} \cdot P_{k}=\frac{1}{2} \sum_{t+s+v=-3} \bigcup_{s}^{\bigcup_{\rho}^{t}} \lambda
$$

(c)

$$
(-I(2)+I(3)) \cdot P_{\lambda-1}=-\bigcup^{\top} \lambda+\frac{1}{2} \sum_{v+s+u=-3} u \bigcup_{\curvearrowright}^{\uparrow v} \lambda
$$

(d)

$$
I_{0} \cdot(-P(2)+P(3))=-\bigcup_{\lambda}+\frac{1}{2} \sum_{t+s+u=-3} u \bigcup_{\downarrow} \lambda
$$

$$
I_{0} \cdot(-P(4)+P(5))=-\frac{1}{2} \bigcirc \mid \bigcup_{\lambda}^{\smile}+\frac{1}{2} \underset{-1}{\downarrow} \oint_{\lambda}^{\bigcup} \lambda .
$$

Now combining Lemma A.23 and Lemma A.24 gives us Proposition A.22, Finally Proposition 4.3 follows by Proposition A.14 and Proposition A.22. This completes the categorification of the SSerre relations. 


\section{REFERENCES}

[B16] H. Bao, Kazhdan-Lusztig theory of super type D and quantum symmetric pairs, Represent. Theory 21 (2017), 247-276.

[BHLW] A. Beliakova, K. Habiro, A. Lauda and B. Webster, Cyclicity for categorified quantum groups, J. Algebra 452 (2016), 118-132.

[BG80] J. Bernstein and S. Gelfand, Tensor products of finite and infinite representations of semisimple Lie algebras, Compos. Math. 41 (1980), 245-285.

[BW89] J. Birman, H. Wenzl, Braids, link polynomials and a new algebra, Trans. Amer. Math. Soc. 313 (1989), 249-273.

[Br16] J. Brundan, On the definition of Kac-Moody 2-category, Math. Ann. 364 (2016), 353-372.

[BKLW] H. Bao, J. Kujawa, Y. Li and W. Wang, Geometric Schur duality of classical type, (with Appendix by Bao, Li and Wang), Transform. Groups (to appear), arXiv:1404.4000v3.

[BLM90] A. Beilinson, G. Lusztig and R. McPherson, A geometric setting for the quantum deformation of $G L_{n}$, Duke Math. J., 61 (1990), 655-677.

[BW13] H. Bao and W. Wang, A new approach to Kazhdan-Lusztig theory of type B via quantum symmetric pairs, to appear in Astérisque. arXiv:1310.0103

[CR08] J. Chuang and R. Rouquier, Derived equivalences for symmetric groups and $\mathfrak{s l}_{2}$-categorification, Ann. of Math. (2) 167 (2008), 245-298.

[D89] V. Deodhar, On some geometric aspects of Bruhat orderings. II. The parabolic analogue of KazhdanLusztig polynomials. J. Algebra 111 (1987), no. 2, 483-506.

[ES13] M. Ehrig and C. Stroppel, Nazarov-Wenzl algebras, coideal subalgebras and categorified skew Howe duality, arXiv:1310.1972

[E13] B. Elias, The two-color Soergel calculus, Compos. Math. 152 (2016), 327-398, arXiv:1308.6611.

[EW13] B. Elias and G. Williamson, Soergel calculus, Represent. Theory 20 (2016), 295-374.

[FKM02] V. Futorny, S. König and V. Mazorchuk, Categories of induced modules and standardly stratified algebras, Alg. Represent. Theory 5 (3) (2002) 259-276.

[FL15] Z. Fan and Y. Li, Geometric Schur duality of classical type, II, Trans. Amer. Math. Soc., Series B2 (2015), 51-92, arXiv: 1408.6740

[FLLLW] Z. Fan, Y. Li, C. Lai, L. Luo and W. Wang, Affine flag varieties and quantum symmetric pairs, Memoirs of AMS (to appear), arXiv:1602.04383.

[GL92] I. Grojnowski and G. Lusztig, On bases of irreducible representations of quantum $G L_{n}$. In: KazhdanLusztig theory and related topics (Chicago, IL, 1989), 167-174, Contemp. Math., 139, Amer. Math. Soc., Providence, RI, 1992.

[Jim86] M. Jimbo, A q-analogue of $U(\mathfrak{g l}(N+1))$, Hecke algebra, and the Yang-Baxter equation, Lett. Math. Phys. 11 (1986), 247-252.

[JMW14] D. Juteau, C. Mautner and G. Williamson, Parity sheaves, J. Amer. Math. Soc. 27 (2014), 1169-1212.

[Ka93] M. Kashiwara, Global crystal bases of quantum groups, Duke Math. J. 69 (1993), 455-485.

[KL09] M. Khovanov and A. Lauda, A diagrammatic approach to categorification of quantum groups. I, Represent. Theory 13 (2009), 309-347.

[KL10] - A categorification of quantum $\mathrm{sl}(n)$, Quantum Topol. 1 (2010), 1-92.

[Ko14] S. Kolb, Quantum symmetric Kac-Moody pairs, Adv. in Math. 267 (2014), 395-469.

[La10] A. Lauda, A categorification of quantum $\mathfrak{s l}(2)$. Adv. Math. 225 (2010), 3327-3424.

[Le03] G. Letzter, Quantum symmetric pairs and their zonal spherical functions, Transformation Groups 8 (2003), 261-292.

[Lib08] N. Libedinsky, Sur la catégorie des bimodules de Soergel. J. Algebra 320 (2008), 2675-2694.

[Lu90] G. Lusztig, Canonical bases arising from quantized enveloping algebras, J. Amer. Math. Soc. 3 (1990), 447-498.

[Lu92] G. Lusztig, Canonical bases in tensor products, Proc. Nat. Acad. Sci. 89 (1992), 8177-8179.

[Lu94] G. Lusztig, Introduction to Quantum Groups, Modern Birkhäuser Classics, Reprint of the 1993 Edition, Birkhäuser, Boston, 2010. 
[LW15] Y. Li and W. Wang, Positivity vs negativity of canonical bases, Proceedings for Lusztig's 70th birthday conference, Bulletin of Institute of Math. Academia Sinica (N.S.), to appear, arXiv:1501.00688,

[MSV13] M. Mackaay, M. Stošić, and P. Vaz, A diagrammatic categorification of the q-Schur algebra, Quantum Topol. 4 (2013), 1-75.

[M87] J. Murakami, The Kauffman polynomial of links and representation theory. Osaka J. Math. 24 (1987), 745-758.

[R08] R. Rouquier, 2-Kac-Moody Algebras, arXiv:0812.5023.

[Soe90] W. Soergel, Kategorie $\mathcal{O}$, perverse Garben und Moduln über den Koinvarianten zur Weylgruppe, J. Amer. Math. Soc. 3 (1990), 421-445.

[Soe92] _ The combinatorics of Harish-Chandra bimodules, J. Reine Angew. Math. 429 (1992), 49-74.

[Soe07] Kazhdan-Lusztig-Polynome und unzerlegbare Bimodulnüber Polynomringen, J. Inst. Math. Jussieu 6 (2007), 501-525.

[Str04] C. Stroppel, A Structure Theorem for Harish-Chandra Bimodules via Coinvariants and Golod Rings, J. of Algebra 282, (2004) 349-367.

[W15] B. Webster, Canonical bases and higher representation theory, Compos. Math. 151 (2015), 121-166.

[W15b] Comparison of canonical bases for Schur and universal enveloping algebras., arXiv:1503.08734.

[Wil11] G. Williamson, Singular Soergel bimodules. Int. Math. Res. Not. IMRN, (20) 4555-4632, 2011.

[Wil08] _ Singular Soergel bimodules, PhD thesis, Albert-Ludwigs-Universität Freiburg, 2008.

(huanchen@math.umd.edu) Department of Mathematics, University of Maryland, College Park, MD 20742, USA

(peng.shan@math.u-psud.fr, pshan@math.tsinghua.edu.cn) LABOratoire DE MathÉMatiques D'Orsay, Univ. Paris-Sud, CNRS, 91405 Orsay, France

Yau Mathematical Sciences Center, Tsinghua University, 100084, Beijing, China

(ww9c@virginia.edu) Department of Mathematics, University of Virginia, Charlottesville, VA 22904, USA

(ben.webster@uwaterloo.ca) Department of Mathematics, University of Virginia, Charlottesville, VA 22904

Current address: Dept. of Pure Mathematics, University of Waterloo \& Perimeter Institute for Theoretical Physics, Waterloo, On, Canada 